\title{
A PERIODIC PARABOLIC CAUCHY PROBLEM: HOMOGENIZATION WITH CORRECTOR
}

\author{
E. S. VASILEVSKAYA
}

\begin{abstract}
A wide class of matrix elliptic second-order differential operators $\mathcal{A}=$ $\mathcal{A}(\mathbf{x}, \mathbf{D})$ with periodic coefficients, acting in $L_{2}\left(\mathbb{R}^{d} ; \mathbb{C}^{n}\right)$, is studied. The operator $\mathcal{A}$ is assumed to admit a factorization of the form $\mathcal{A}=\mathcal{X}^{*} \mathcal{X}$, where $\mathcal{X}$ is a homogeneous first-order differential operator. An approximation for the operator exponential $e^{-\mathcal{A} \tau}$ as $\tau \rightarrow \infty$ in the $\left(L_{2}\left(\mathbb{R}^{d} ; \mathbb{C}^{n}\right)\right)$-operator norm is obtained, with error estimate of the order of $\tau^{-1}$. In the approximation, a corrector is taken into account. The result is applied to the study of homogenization for solutions of the Cauchy problem $\partial_{\tau} \mathbf{u}_{\varepsilon}=-\mathcal{A}_{\varepsilon} \mathbf{u}_{\varepsilon}$, where $\mathcal{A}_{\varepsilon}=\mathcal{A}(\mathbf{x} / \varepsilon, \mathbf{D})$. An approximation with corrector for $\mathbf{u}_{\varepsilon}$ in the $\left(L_{2}\left(\mathbb{R}^{d} ; \mathbb{C}^{n}\right)\right)$-norm is obtained for fixed $\tau>0$, with error estimate of the order of $\varepsilon^{2}$.
\end{abstract}

\section{$\S 0$. INTRODUCTION}

0.1. An extensive literature is devoted to homogenization problems for periodic differential operators (DO's) in the small period limit (see, e.g., the books 1, 2, 10). Special attention has been paid to homogenization for solutions of parabolic equations. Particularly, we note the paper [8], where the asymptotics for large times of the parabolic Green function was obtained by the spectral method.

Traditional methods give convergence (in some sense) for solutions of an equation with rapidly oscillating coefficients to the solution of the corresponding homogenized problem. Usually, error estimates are obtained under some smoothness conditions on the problem data.

In [3, 4, 5, 6, 7] and 14, 15, a spectral method was developed, which provides a way for obtaining order-sharp error estimates for approximations in the $\left(L_{2}\left(\mathbb{R}^{d}\right)\right)$-norm in homogenization problems. The present paper continues these investigations. It concerns homogenization for solutions of the Cauchy problem for the parabolic equation $\partial_{\tau} \mathbf{u}_{\varepsilon}=$ $-\mathcal{A}_{\varepsilon} \mathbf{u}_{\varepsilon}$. Here $\mathcal{A}=\mathcal{A}(\mathbf{x}, \mathbf{D})$ is a matrix elliptic second-order operator with periodic coefficients acting in $L_{2}\left(\mathbb{R}^{d} ; \mathbb{C}^{n}\right)$, and $\mathcal{A}_{\varepsilon}=\mathcal{A}(\mathbf{x} / \varepsilon, \mathbf{D})$.

0.2. The class of operators. We start with the description of the class of elliptic operators. We consider DO's in $L_{2}\left(\mathbb{R}^{d} ; \mathbb{C}^{n}\right)$ of the form

$$
\mathcal{A}=\mathcal{A}(g, f)=f(\mathbf{x})^{*} b(\mathbf{D})^{*} g(\mathbf{x}) b(\mathbf{D}) f(\mathbf{x}) .
$$

Here $g(\mathbf{x})$ is an $(m \times m)$-matrix, and $f(\mathbf{x})$ is an $(n \times n)$-matrix. It is assumed that $m \geq n$, the matrix-valued functions $g(\mathbf{x})$ and $f(\mathbf{x})$ are periodic with respect to some lattice $\Gamma \subset \mathbb{R}^{d}$, and

$$
g(x)>0, \quad g, g^{-1} \in L_{\infty}, \quad f, f^{-1} \in L_{\infty} .
$$

2000 Mathematics Subject Classification. Primary 35B27, 35K30.

Key words and phrases. Parabolic Cauchy problem, homogenization, effective operator, corrector. Supported by RFBR (grant no. 08-01-00209-a) and by a "Scientific schools" grant (no. 816.2008.1). 
Let $\Omega$ denote the elementary cell of the lattice $\Gamma$. Next, $b(\mathbf{D})$ is an $(m \times n)$-matrix firstorder DO such that $\operatorname{rank} b(\boldsymbol{\xi})=n, 0 \neq \boldsymbol{\xi} \in \mathbb{R}^{d}$. In the case where $f(\mathbf{x})=\mathbf{1}_{n}$, we use the notation

$$
\widehat{\mathcal{A}}=\widehat{\mathcal{A}}(g)=b(\mathbf{D})^{*} g(\mathbf{x}) b(\mathbf{D}) .
$$

We introduce a small parameter $\varepsilon>0$. For an arbitrary $\Gamma$-periodic function $\phi(\mathbf{x})$, we denote

$$
\phi^{\varepsilon}(\mathbf{x})=\phi\left(\varepsilon^{-1} \mathbf{x}\right), \quad \varepsilon>0 .
$$

Put

$$
\mathcal{A}_{\varepsilon}=\mathcal{A}\left(g^{\varepsilon}, f^{\varepsilon}\right), \quad \widehat{\mathcal{A}}_{\varepsilon}=\widehat{\mathcal{A}}\left(g^{\varepsilon}\right) .
$$

The coefficients of the operators $\mathcal{A}_{\varepsilon}, \widehat{\mathcal{A}}_{\varepsilon}$ oscillate rapidly as $\varepsilon \rightarrow 0$.

0.3. Operator estimates in homogenization theory. In 3, 4, 5, 6, 7, the homogenization problem for solutions of elliptic equations with the operators $(0.3)$ was studied. This problem reduces to the investigation of the behavior of the resolvent $\left(\mathcal{A}_{\varepsilon}+I\right)^{-1}$ as $\varepsilon \rightarrow 0$. In [3, 4], an estimate of the form

$$
\left\|\left(\hat{\mathcal{A}}_{\varepsilon}+I\right)^{-1}-\left(\hat{\mathcal{A}}^{0}+I\right)^{-1}\right\|_{L_{2}\left(\mathbb{R}^{d}\right) \rightarrow L_{2}\left(\mathbb{R}^{d}\right)} \leq C \varepsilon
$$

was obtained for the first time. Here $\hat{\mathcal{A}}^{0}=b(\mathbf{D})^{*} g^{0} b(\mathbf{D})$ is the effective operator with a constant effective matrix $g^{0}$. (The procedure of constructing the effective matrix is described in $\S 5$.) Later, in [5, 6], a more accurate approximation was obtained for the resolvent of the operator $\hat{\mathcal{A}}_{\varepsilon}$ :

$$
\left\|\left(\hat{\mathcal{A}}_{\varepsilon}+I\right)^{-1}-\left(\hat{\mathcal{A}}^{0}+I\right)^{-1}-\varepsilon K(\varepsilon)\right\|_{L_{2}\left(\mathbb{R}^{d}\right) \rightarrow L_{2}\left(\mathbb{R}^{d}\right)} \leq C \varepsilon^{2} .
$$

Here $K(\varepsilon)$ is the so-called corrector. In [7], the following approximation for the resolvent of the operator $\hat{\mathcal{A}}_{\varepsilon}$ in the norm of the space of operators acting from $L_{2}\left(\mathbb{R}^{d} ; \mathbb{C}^{n}\right)$ to the Sobolev class $H^{1}\left(\mathbb{R}^{d} ; \mathbb{C}^{n}\right)$ was found:

$$
\left\|\left(\widehat{\mathcal{A}}_{\varepsilon}+I\right)^{-1}-\left(\hat{\mathcal{A}}^{0}+I\right)^{-1}-\varepsilon K_{1}(\varepsilon)\right\|_{L_{2}\left(\mathbb{R}^{d}\right) \rightarrow H^{1}\left(\mathbb{R}^{d}\right)} \leq C \varepsilon .
$$

Here $K_{1}(\varepsilon)$ is the traditional corrector arising in the classical homogenization theory. Note that the corrector $K(\varepsilon)$ in $(0.5)$ consists of three terms:

$$
K(\varepsilon)=K_{1}(\varepsilon)+K_{1}(\varepsilon)^{*}+K_{3},
$$

with $K_{3}$ independent of $\varepsilon$. Estimates (0.4)-(0.6) are order sharp, and the constants in the estimates are controlled explicitly in terms of the problem data. In [4, 5, 6, 7, some similar (but slightly more complicated) approximations for the resolvent $\left(\mathcal{A}_{\varepsilon}+I\right)^{-1}$ were obtained in the case where $f \neq \mathbf{1}_{n}$.

The approach developed in [3, 4, 5, 6, 7] for elliptic problems was applied also to the homogenization of parabolic problems. The problem then reduces to the study of the operator exponential $e^{-\mathcal{A}_{\varepsilon} \tau}, \tau>0$. The following estimate was obtained in [14, 15]:

$$
\left\|e^{-\hat{\mathcal{A}}_{\varepsilon} \tau}-e^{-\hat{\mathcal{A}}^{0} \tau}\right\|_{L_{2}\left(\mathbb{R}^{d}\right) \rightarrow L_{2}\left(\mathbb{R}^{d}\right)} \leq C \varepsilon\left(\tau+\varepsilon^{2}\right)^{-1 / 2}, \quad \varepsilon>0, \quad \tau \geq 0 .
$$

(In [14, the statements were described in detail, and the full presentation was given in [15].) A similar but slightly more complicated estimate was obtained for $e^{-\mathcal{A}_{\varepsilon} \tau}$ in the case where $f \neq \mathbf{1}_{n}$. The problem of approximation of the operator exponential $e^{-\mathcal{A}_{\varepsilon} \tau}$ in the $\left(L_{2}\left(\mathbb{R}^{d} ; \mathbb{C}^{n}\right)\right)$-operator norm, with corrector taken into account, has not been studied earlier.

The approach developed in [3, 4, 5, 6, 7] for elliptic problems and in [14, 15] for parabolic problems can be called a spectral approach. The homogenization procedure 
is studied as a spectral threshold effect near the bottom of the spectrum of a periodic operator $\mathcal{A}$. (The bottom of the spectrum of the operators $(0.1),(0.2)$ is the point $\lambda=0$.) It is essential that, for obtaining approximations in the $L_{2}\left(\mathbb{R}^{d} ; \mathbb{C}^{n}\right)$-operator norm, a scaling transformation can be used. The study of the operators $\left(\mathcal{A}_{\varepsilon}+I\right)^{-1}, e^{-\mathcal{A}_{\varepsilon} \tau}$ reduces to that of the operators $\left(\mathcal{A}+\varepsilon^{2} I\right)^{-1}$ and $\exp \left(-\mathcal{A} \varepsilon^{-2} \tau\right)$, respectively. The behavior of the latter pair of operators can be described in terms of threshold characteristics of $\mathcal{A}$ (the spectral characteristics near the bottom of the spectrum). Then a considerable part of the constructions can be carried out in an operator-theoretic framework.

Another (nonspectral) method of obtaining operator estimates in homogenization theory was suggested by V. V. Zhikov. His method is related to the analysis of the first approximation to the solution and incorporating an additional parameter (a shift by an arbitrary vector $\boldsymbol{\omega} \in \Omega$ ). In 9, 11, estimates $(0.4),(0.6)$ for the acoustic operator $(\hat{\mathcal{A}}=-\operatorname{div} g(\mathbf{x}) \nabla)$ and the operator of elasticity theory were obtained by that method. In [12, estimate (0.8) for the acoustic operator was obtained, as well as the following approximation:

$$
\left\|e^{-\hat{\mathcal{A}}_{\varepsilon} \tau}-e^{-\hat{\mathcal{A}}^{0} \tau}-\varepsilon \widehat{\mathcal{K}}_{1}(\varepsilon, \tau)\right\|_{L_{2}\left(\mathbb{R}^{d}\right) \rightarrow H^{1}\left(\mathbb{R}^{d}\right)} \leq C \varepsilon .
$$

0.4. Main results. In the present paper we consider the problem of approximation of the operator exponential $e^{-\mathcal{A}_{\varepsilon} \tau}, \tau>0$, as $\varepsilon \rightarrow 0$. Our goal is to obtain approximation for the operator $e^{-\mathcal{A}_{\varepsilon} \tau}$ in the $L_{2}\left(\mathbb{R}^{d} ; \mathbb{C}^{n}\right)$-operator norm (for fixed $\tau>0$ ) with error estimate of the order of $\varepsilon^{2}$. For the simpler operator $\widehat{\mathcal{A}}_{\varepsilon}$, the following result is established (Theorem 8.2):

$$
\left\|e^{-\hat{\mathcal{A}}_{\varepsilon} \tau}-e^{-\hat{\mathcal{A}}^{0} \tau}-\varepsilon \hat{\mathcal{K}}(\varepsilon, \tau)\right\|_{L_{2}\left(\mathbb{R}^{d}\right) \rightarrow L_{2}\left(\mathbb{R}^{d}\right)} \leq \widehat{C} \varepsilon^{2}\left(\tau+\varepsilon^{2}\right)^{-1}, \quad \varepsilon>0, \quad \tau \geq 0 .
$$

The approximation (0.9) is sharper than (0.8). This refinement is achieved by introducing a corrector $\hat{\mathcal{K}}(\varepsilon, \tau)$. In the case of the operator $\mathcal{A}_{\varepsilon}$ (if $f \neq \mathbf{1}_{n}$ ), an approximation for the "bordered" operator exponential is found (Theorem 8.1):

$$
\begin{array}{r}
\left\|f^{\varepsilon} e^{-\mathcal{A}_{\varepsilon} \tau}\left(f^{\varepsilon}\right)^{*}-f_{0} e^{-\mathcal{A}^{0} \tau} f_{0}-\varepsilon \mathcal{K}(\varepsilon, \tau)\right\|_{L_{2}\left(\mathbb{R}^{d}\right) \rightarrow L_{2}\left(\mathbb{R}^{d}\right)} \leq C \varepsilon^{2}\left(\tau+\varepsilon^{2}\right)^{-1}, \\
\varepsilon>0, \quad \tau \geq 0 .
\end{array}
$$

Here $\mathcal{A}^{0}=f_{0} b(\mathbf{D})^{*} g^{0} b(\mathbf{D}) f_{0}=f_{0} \hat{\mathcal{A}}^{0} f_{0}$ and $f_{0}=|\Omega|^{-1} \int_{\Omega}\left(f(\mathbf{x}) f(\mathbf{x})^{*}\right)^{-1} d \mathbf{x}$. It turns out that the corrector $\mathcal{K}(\varepsilon, \tau)$ contains three terms, two of them being adjoint to each other and involving rapidly oscillating factors. The first term of the corrector corresponds to the traditional corrector. The third term does not involve variable coefficients, and for $\tau \geq \varepsilon^{2}$ does not depend on $\varepsilon$. In the structure of the corrector $\mathcal{K}(\varepsilon, \tau)$, an obvious analogy is seen with the result for the resolvent $\left(\mathcal{A}_{\varepsilon}+I\right)^{-1}$, obtained in [6] (see the discussion below in Subsection 8.4).

The results obtained are applied to homogenization for the Cauchy problem

$$
\begin{array}{r}
Q^{\varepsilon}(\mathbf{x}) \partial_{\tau} \mathbf{u}_{\varepsilon}(\mathbf{x}, \tau)=-\widehat{\mathcal{A}}_{\varepsilon} \mathbf{u}_{\varepsilon}(\mathbf{x}, \tau), \quad Q^{\varepsilon}(\mathbf{x}) \partial_{\tau} \mathbf{u}_{\varepsilon}(\mathbf{x}, 0)=\phi(\mathbf{x}), \\
\mathbf{x} \in \mathbb{R}^{d}, \quad \tau \in(0, T) .
\end{array}
$$

Here $0<T \leq \infty$ and $\phi \in L_{2}\left(\mathbb{R}^{d} ; \mathbb{C}^{n}\right)$. The matrix $Q(\mathbf{x})$ is positive definite and periodic. Let $\mathbf{u}_{0}(\mathbf{x}, \tau)$ be the solution of the "homogenized" problem

$$
\bar{Q} \partial_{\tau} \mathbf{u}_{0}(\mathbf{x}, \tau)=-\widehat{\mathcal{A}}^{0} \mathbf{u}_{0}(\mathbf{x}, \tau), \quad \bar{Q} \mathbf{u}_{0}(\mathbf{x}, 0)=\phi(\mathbf{x}) .
$$

Here $\bar{Q}$ is the mean value of the matrix $Q$. In the case where $Q=\mathbf{1}_{n}$, approximation for $\mathbf{u}_{\varepsilon}$ follows from (0.9), and in the general case it follows from (0.10). Here $f(\mathbf{x})$ is a 
$\Gamma$-periodic $(n \times n)$-matrix such that $Q(\mathbf{x})=\left(f(\mathbf{x}) f(\mathbf{x})^{*}\right)^{-1}$. For the solutions $\mathbf{u}_{\varepsilon}$ of the problem (0.11) we find:

$$
\begin{array}{r}
\left\|\mathbf{u}_{\varepsilon}(\cdot, \tau)-\mathbf{u}_{0}(\cdot, \tau)-\varepsilon \mathbf{u}_{\varepsilon}^{(1)}(\cdot, \tau)\right\|_{L_{2}\left(\mathbb{R}^{d}\right)} \leq C \varepsilon^{2}\left(\tau+\varepsilon^{2}\right)^{-1}\|\phi\|_{L_{2}\left(\mathbb{R}^{d}\right)}, \\
\varepsilon>0, \quad \tau \geq 0 .
\end{array}
$$

Here $\mathbf{u}_{\varepsilon}^{(1)}(\cdot, \tau)=\mathcal{K}(\varepsilon, \tau) \phi$. We also study the behavior of solutions of the nonhomogeneous Cauchy problem

$$
Q^{\varepsilon}(\mathbf{x}) \partial_{\tau} \mathbf{u}_{\varepsilon}(\mathbf{x}, \tau)=-\widehat{\mathcal{A}}_{\varepsilon} \mathbf{u}_{\varepsilon}(\mathbf{x}, \tau)+\mathbf{F}(\mathbf{x}, \tau), \quad Q\left(\varepsilon^{-1} \mathbf{x}\right) \mathbf{u}_{\varepsilon}(\mathbf{x}, 0)=\phi(\mathbf{x}) .
$$

Via the scaling transformation, the problem of approximation of the operator exponential $e^{-\mathcal{A}_{\varepsilon} \tau}$ in the $L_{2}\left(\mathbb{R}^{d} ; \mathbb{C}^{n}\right)$-operator norm for small $\varepsilon>0$ reduces to the study of the behavior of the operator $\exp \left(-\mathcal{A} \varepsilon^{-2} \tau\right)$. Thus, we need to study the exponential $e^{-\mathcal{A} \tau}$ as $\tau \rightarrow \infty$. In [14, 15], the leading term of the approximation was found:

$$
\left\|f e^{-\mathcal{A} \tau} f^{*}-f_{0} e^{-\mathcal{A}^{0} \tau} f_{0}\right\|_{L_{2}\left(\mathbb{R}^{d}\right) \rightarrow L_{2}\left(\mathbb{R}^{d}\right)} \leq C(1+\tau)^{-1 / 2}, \quad \tau \geq 0 .
$$

Now we refine this approximation (see Theorem 7.1) by introducing a corrector $\mathfrak{K}(\tau)$ of order $\tau^{-1 / 2}$ :

$$
\left\|f e^{-\mathcal{A} \tau} f^{*}-f_{0} e^{-\mathcal{A}^{0} \tau} f_{0}-\mathfrak{K}(\tau)\right\|_{L_{2}\left(\mathbb{R}^{d}\right) \rightarrow L_{2}\left(\mathbb{R}^{d}\right)} \leq C(1+\tau)^{-1}, \quad \tau \geq 0 .
$$

We note that estimates $(0.15)$ and $(0.16)$ are of independent interest: they show that the solution of the parabolic Cauchy problem with periodic coefficients is close at large times to the solution of the "effective" problem with constant coefficients.

0.5. Outline of the method. In order to obtain the approximation (0.16), we expand the operator $\mathcal{A}$ into the direct integral of operators $\mathcal{A}(\mathbf{k})$ acting in $L_{2}\left(\Omega ; \mathbb{C}^{n}\right)$ and depending on the parameter $\mathbf{k} \in \mathbb{R}^{\mathbf{d}}$ (the quasimomentum). The operator $\mathcal{A}(\mathbf{k})$ is defined by the differential expression $\mathcal{A}(\mathbf{x}, \mathbf{D}+\mathbf{k})$ with periodic boundary conditions. The spectrum of the operators $\mathcal{A}(\mathbf{k})$ is discrete. The operator family $\mathcal{A}(\mathbf{k})$ is studied by methods of analytic perturbation theory with respect to the one-dimensional parameter $t=|\mathbf{k}|$. Moreover, we should make our estimates uniform with respect to the parameter $\boldsymbol{\theta}=t^{-1} \mathbf{k}$. The operator family $\mathcal{A}(\mathbf{k})=A(t, \boldsymbol{\theta})$ is described in terms of the abstract operator-theoretic approach suggested in the papers [3, 4, 5, 6, 7, 14, 15.

At the abstract level, we consider selfadjoint operator families of the form $A(t)=$ $X(t)^{*} X(t)$ in a Hilbert space $\mathfrak{H}$. Here $X(t)=X_{0}+t X_{1}, t \in \mathbb{R}$, where $X_{0}: \mathfrak{H} \rightarrow \mathfrak{H}_{*}$ is a closed and densely defined operator and $X_{1}: \mathfrak{H} \rightarrow \mathfrak{H}_{*}$ is a bounded operator. The point $\lambda=0$ is assumed to be an isolated eigenvalue of the operator $A(0)$ of multiplicity $n$. The notion of the spectral germ $S$ of the operator family $A(t)$ plays a crucial role in our investigation. The germ $S$ is a selfadjoint operator acting in the $n$-dimensional space $\mathfrak{N}=\operatorname{Ker} A(0)$. The spectral germ contains information about threshold characteristics of the operator near the bottom of the spectrum. In terms of the spectral germ, one can find an approximation for the operator exponential $e^{-A(t) \tau}$ :

$$
\left\|e^{-A(t) \tau}-e^{-t^{2} S P \tau}\right\|_{\mathfrak{H} \rightarrow \mathfrak{H}} \leq C(1+\tau)^{-1 / 2}, \quad \tau \geq 0, \quad|t| \leq t^{0},
$$

where $P$ is the orthogonal projection onto $\mathfrak{N}$, and $C$ and $t^{0}$ are controllable constants. Estimate (0.17) was obtained in [15]. Here we find a sharper approximation:

$$
\left\|e^{-A(t) \tau}-e^{-t^{2} S P \tau}-K(t, \tau)\right\|_{\mathfrak{H} \rightarrow \mathfrak{H}} \leq C(1+\tau)^{-1}, \quad \tau \geq 0, \quad|t| \leq t^{0} .
$$

The definition of the corrector $K(t, \tau)$ requires the introduction of two operators of finite rank, $Z$ and $N$; the expressions for them are given in $\S 1$. Estimate $(0.18)$ is the main 
result of the abstract part of the paper. Application of estimate (0.18) to the differential operators $A(t, \boldsymbol{\theta})=\mathcal{A}(\mathbf{k})$ leads to required results for the operator exponential.

0.6. The structure of the paper. The paper consists of three chapters. In Chapter 1 $(\S \S 1-3)$ some facts from [4, 5] are given, in relation to the abstract approach. Also, an approximation with corrector for the operator exponential at the abstract level is obtained there (Theorem 2.1), as well as an approximation for the "bordered" operator exponential, which is the basis for further considerations. In Chapter $2(\S \S 4-7)$, a description is given of the class of operators under study and the necessary information from [4] is presented about incorporation of periodic DO's into the abstract framework. The results of Chapter 1 are applied to the approximation of the "bordered" operator exponential $f e^{-\mathcal{A} \tau} f^{*}$ as $\tau \rightarrow \infty$ (Theorem 7.1). In Chapter 3 ( $\left.\S \S 8-10\right)$, we use the scaling transformation to obtain an approximation of the operator $f^{\varepsilon} e^{-\mathcal{A}_{\varepsilon} \tau}\left(f^{\varepsilon}\right)^{*}$ as $\varepsilon \rightarrow 0$. This is the main result of the paper (Theorem 8.1). Finally, Theorem 8.1 is applied to homogenization for the homogeneous Cauchy problem (0.11), as well as for the nonhomogeneous Cauchy problem (0.14).

0.7. Notation. Let $\mathfrak{H}, \mathfrak{H}_{*}$ be separable Hilbert spaces. The symbols $(\cdot, \cdot)_{\mathfrak{H}}$ and $\|\cdot\|_{\mathfrak{H}}$ stand for the inner product and the norm in $\mathfrak{H}$, respectively. The symbol $\|\cdot\|_{\mathfrak{H} \rightarrow \mathfrak{H}_{*}}$ stands for the norm of a bounded operator acting from $\mathfrak{H}$ to $\mathfrak{G}$. Sometimes we omit indices in the notation of the norm and inner product. Let $I=I_{\mathfrak{H}}$ denote the identity operator in $\mathfrak{H}$. If $A: \mathfrak{H} \rightarrow \mathfrak{H}_{*}$ is a linear operator, then Dom $A$ denotes the domain of $A$. The spectrum of a closed operator $T$ in $\mathfrak{H}$ is denoted by spec $T$. If $\mathfrak{N}$ is a subspace in $\mathfrak{H}$, then $\mathfrak{N}^{\perp}:=\mathfrak{H} \ominus \mathfrak{N}$. If $P$ is the orthogonal projection $\mathfrak{H}$ onto $\mathfrak{N}$, then $P^{\perp}$ is the orthogonal projection onto $\mathfrak{N}^{\perp}$. The symbols $\langle\cdot, \cdot\rangle$ and $|\cdot|$ stand for the standard inner product and the standard norm of a vector in $\mathbb{C}^{n}$, respectively. By $\mathbf{1}_{n}$ we denote the unit $(n \times n)$-matrix; $\mathbf{x}=\left(x^{1}, \ldots, x^{d}\right) \in \mathbb{R}^{d}, i D_{j}=\partial_{j}=\partial / \partial x^{j}, j=1, \ldots, d$; $\nabla=\operatorname{grad}=\left(\partial_{1}, \ldots, \partial_{d}\right), \mathbf{D}=-i \nabla=\left(D_{1}, \ldots, D_{d}\right)$. For an $(m \times n)$-matrix $b$, we write $|b|$ for the norm of the corresponding linear operator from $\mathbb{C}^{n}$ to $\mathbb{C}^{m}$, and $b^{*}$ is the Hermitian adjoint $(n \times m)$-matrix. The $L_{p}$-space of $\mathfrak{H}$-valued functions on the interval $(0, T)$ is denoted by $L_{p}((0, T) ; \mathfrak{H})=: \mathcal{H}_{p}, 1 \leq p \leq \infty$. The vector Sobolev classes of order $s$ in a domain $\mathcal{O} \subseteq \mathbb{R}^{d}$ are denoted by $H^{s}\left(\mathcal{O} ; \mathbb{C}^{n}\right)$.

0.8. The author is grateful to T. A. Suslina for setting the problem and guidance. The author is also grateful to M. Sh. Birman for attention to the work and helpful remarks.

\section{Chapter 1. ABSTRACT FRAMEWORK}

\section{§1. Preliminaries}

The material of this section is borrowed from [4, 5].

1.1. Operator pencils of the form $X(t)^{*} X(t)$. Let $\mathfrak{H}, \mathfrak{H}_{*}$ be complex separable Hilbert spaces. Suppose that $X_{0}: \mathfrak{H} \rightarrow \mathfrak{H}_{*}$ is a closed and densely defined operator and that $X_{1}: \mathfrak{H} \rightarrow \mathfrak{H}_{*}$ is a bounded operator. We introduce an operator family $X(t)=X_{0}+$ $t X_{1}, t \in \mathbb{R}$. Then $X(t)$ is a closed and densely defined operator on $\operatorname{Dom} X(t)=\operatorname{Dom} X_{0}$. We have $X(t)^{*}=X_{0}^{*}+t X_{1}^{*}$ on Dom $X_{0}^{*}$. The expression $A(t):=X(t)^{*} X(t)$ determines a selfadjoint positive operator family in the space $\mathfrak{H}$.

The operator $A(t)$ corresponds to the closed quadratic form $a(t)[u, u]=\|X(t) u\|_{\mathfrak{H}_{*}}^{2}$, $u \in \operatorname{Dom} X_{0}$. We denote $A_{0}:=A(0)=X_{0}^{*} X_{0}$,

$$
\mathfrak{N}:=\operatorname{Ker} A_{0}=\operatorname{Ker} X_{0}, \quad \mathfrak{N}_{*}:=\operatorname{Ker} X_{0}^{*},
$$

and suppose that $0<n:=\operatorname{dim} \mathfrak{N}<\infty, n \leq n_{*}:=\operatorname{dim} \mathfrak{N}_{*} \leq \infty$. Let $P$ be the orthogonal projection of $\mathfrak{H}$ onto $\mathfrak{N}$ and $P_{*}$ the orthogonal projection of $\mathfrak{H}_{*}$ onto $\mathfrak{N}_{*}$. Also, we assume 
that the point $\lambda=0$ is an isolated point of the spectrum of $A_{0}$. Let $d_{0}$ be the distance from the point $\lambda=0$ to the rest of the spectrum of $A_{0}$. We fix a number $\delta>0$ such that $8 \delta<d_{0}$. Let $F(t, s)$ denote the spectral projection of the operator $A(t)$ for the interval $[0, s]$, and let $\mathfrak{F}(t, s):=F(t, s) \mathfrak{H}$. It turns out that for

$$
|t| \leq t^{0}:=\delta^{1 / 2}\left\|X_{1}\right\|^{-1}
$$

we have $\operatorname{dim} \mathfrak{F}(t, \delta)=\operatorname{dim} \mathfrak{F}(t, 3 \delta)=n$ (see [4, Proposition 1.1.2]). If $s=\delta$, we use the notation $F(t):=F(t, \delta)$.

1.2. The operators $Z$ and $R$. Given $z \in \mathfrak{H}_{*}$, we consider the following equation for an element $\psi \in \operatorname{Dom} X_{0} \cap \mathfrak{N}^{\perp}: X_{0}^{*}\left(X_{0} \psi-z\right)=0$. This equation is understood in a weak sense, i.e.,

$$
\left(X_{0} \psi, X_{0} \zeta\right)_{\mathfrak{H}_{*}}=\left(z, X_{0} \zeta\right)_{\mathfrak{H}_{*}}, \quad \forall \zeta \in \operatorname{Dom} X_{0} \cap \mathfrak{N}^{\perp}
$$

As was mentioned in [4, Subsection 1.1.2], a solution $\psi$ exists and is unique for each $z \in \mathfrak{H}_{*}$. We put $z=-X_{1} \omega, \omega \in \mathfrak{N}$, and denote by $\psi(\omega)$ the element $\psi$ that satisfies (1.2) (for such a $z$ ). We define a continuous linear operator $Z: \mathfrak{H} \rightarrow \mathfrak{H}$ by the formulas

$$
\begin{array}{ll}
Z \omega=\psi(\omega), & \omega \in \mathfrak{N}, \\
Z x=0, & x \in \mathfrak{N}^{\perp} .
\end{array}
$$

Observe that

$$
P Z=0, \quad Z^{*} P=0 .
$$

Put $\omega_{*}:=X_{0} \psi(\omega)+X_{1} \omega \in \mathfrak{N}_{*}$. Then $\omega_{*}=P_{*} X_{1} \omega$. We introduce a linear operator $R: \mathfrak{N} \rightarrow \mathfrak{N}_{*}$ by the formula

$$
R \omega=\omega_{*}
$$

In other words, $R=\left.P_{*} X_{1}\right|_{\mathfrak{N}}$.

1.3. The spectral germ $S$. The following definition was introduced in [4, Subsection 1.1.3].

Definition 1.1. The spectral germ of the operator family $A(t)$ at $t=0$ is the operator

$$
S:=R^{*} R: \mathfrak{N} \rightarrow \mathfrak{N} .
$$

The operator $S$ is selfadjoint and admits the representation $S=\left.P X_{1}^{*} P_{*} X_{1}\right|_{\mathfrak{N}}$. The spectral germ $S$ is said to be nondegenerate if $\operatorname{Ker} S=0$ (or equivalently, $\operatorname{rank} R=n$ ).

Let $\widetilde{A}(t)$ be another operator family in $\mathfrak{H}$ of the form

$$
\widetilde{A}(t)=\tilde{X}(t)^{*} \tilde{X}(t), \quad \tilde{X}(t)=\tilde{X}_{0}+t \tilde{X}_{1}: \mathfrak{H} \rightarrow \tilde{\mathfrak{H}}_{*} .
$$

It is assumed that $\widetilde{A}(t)$ is subject to the same conditions as $A(t)$. If $\mathfrak{N}=\tilde{\mathfrak{N}}$ and $S=\widetilde{S}$, then we say that the families $A(t)$ and $\widetilde{A}(t)$ are threshold equivalent.

By the general analytic perturbation theory, for $|t| \leq t^{0}$ there exist real-analytic functions $\lambda_{1}(t), \ldots, \lambda_{n}(t)$ and real-analytic $\mathfrak{H}$-valued functions $\varphi_{1}(t), \ldots, \varphi_{n}(t)$ such that

$$
A(t) \varphi_{l}(t)=\lambda_{l}(t) \varphi_{l}(t), \quad l=1, \ldots, n,
$$

and the family $\left\{\varphi_{l}(t)\right\}_{l=1}^{n}$ is an orthogonal basis in the space $\mathfrak{F}(t, \delta)$. If $t_{*}$ is sufficiently small, then for $|t| \leq t_{*}\left(\leq t^{0}\right)$ we have the following convergent power series expansions:

$$
\begin{aligned}
\varphi_{l}(t) & =\omega_{l}+t \varphi_{l}^{(1)}+t^{2} \varphi_{l}^{(2)}+\cdots, & & l=1, \ldots, n, \\
\lambda_{l}(t) & =\gamma_{l} t^{2}+\mu_{l} t^{3}+\cdots, & & \gamma_{l} \geq 0, \quad \mu_{l} \in \mathbb{R}, \quad l=1, \ldots, n .
\end{aligned}
$$


The elements $\omega_{1}, \ldots, \omega_{n}$ form an orthogonal basis in $\mathfrak{N}$. In [4, Proposition 1.1.6] it was shown that the numbers $\gamma_{l}$ and the elements $\omega_{l}$ are eigenvalues and eigenvectors of the spectral germ $S$, i.e.,

$$
S \omega_{l}=\gamma_{l} \omega_{l}, \quad l=1, \ldots, n .
$$

Relations (1.7) give an independent (spectral) definition of the germ $S$. The nondegeneracy of the spectral germ is equivalent to the fact that

$$
\gamma_{l} \geq c_{*}>0, \quad l=1, \ldots, n .
$$

1.4. Threshold approximations for the operators $F(t)$ and $A(t) F(t)$. In [4, Chapter $1, \S 4]$ the following result was obtained:

$$
F(t)-P=\Phi(t), \quad\|\Phi(t)\| \leq C_{1}|t|, \quad C_{1}:=\beta_{1} \delta^{-1 / 2}\left\|X_{1}\right\|, \quad|t| \leq t^{0} .
$$

In what follows we denote by $\beta, \beta_{1}, \beta_{2}, \beta^{(2)}$ absolute constants. Their values can be controlled explicitly. In [4, $\$ 2$ ] the above result was improved:

$$
F(t)=P+t F_{1}+F_{2}(t), \quad|t| \leq t^{0},
$$

where

$$
\left\|F_{2}(t)\right\| \leq \beta^{(2)} \delta^{-1}\left\|X_{1}\right\|^{2} t^{2}, \quad|t| \leq t^{0},
$$

and the operator $F_{1}$ can be found from the expansions (1.5), (1.6):

$$
F_{1}=\sum_{l=1}^{n}\left(\left(\cdot, \varphi_{l}^{(1)}\right)_{\mathfrak{H}} \omega_{l}+\left(\cdot, \omega_{l}\right)_{\mathfrak{H}} \varphi_{l}^{(1)}\right)
$$

In [5, Theorem 2.2], it was shown that the operator $F_{1}$ is given by the formula

$$
F_{1}=Z P+P Z^{*} \text {. }
$$

In [4. Chapter 1, §4], the following approximation for the operator $A(t) F(t)$ was obtained:

$$
A(t) F(t)-t^{2} S P=\Psi(t), \quad\|\Psi(t)\| \leq C_{2}|t|^{3}, \quad C_{2}:=\beta_{2} \delta^{-1 / 2}\left\|X_{1}\right\|^{3}, \quad|t| \leq t^{0} .
$$

To refine this approximation, we should take into account the term $\mu_{l} t^{3}$ in (1.6) and the term $t \varphi_{l}^{(1)}$ in (1.5). By Theorem 4.1 in [5],

$$
A(t) F(t)=t^{2} S P+t^{3} K+\widetilde{\Psi}(t) \text { for }|t| \leq t^{0},
$$

where $K$ is a continuous operator independent of $t$, and for $\widetilde{\Psi}(t)$ the following estimate is valid:

$$
\|\widetilde{\Psi}(t)\| \leq \beta \delta^{-1}\left\|X_{1}\right\|^{4} t^{4} .
$$

In [5, §4], an explicit expression for the operator $K$ was obtained from the expansions $(1.5),(1.6)$ :

$$
\begin{aligned}
K & =K_{0}+N_{0}+N_{*}, \quad N_{0}+N_{*}=N, \\
K_{0} & =\sum_{l=1}^{n} \gamma_{l}\left(\left(\cdot, \psi_{l}\right)_{\mathfrak{H}} \omega_{l}+\left(\cdot, \omega_{l}\right)_{\mathfrak{H}} \psi_{l}\right), \\
N_{0} & =\sum_{l=1}^{n} \mu_{l}\left(\cdot, \omega_{l}\right)_{\mathfrak{H}} \omega_{l}, \quad N_{*}=\sum_{l=1}^{n} \gamma_{l}\left(\left(\cdot, \widetilde{\omega}_{l}\right)_{\mathfrak{H}} \omega_{l}+\left(\cdot, \omega_{l}\right)_{\mathfrak{H}} \tilde{\omega}_{l}\right),
\end{aligned}
$$

where $\psi_{l}:=\psi\left(\omega_{l}\right)=Z \omega_{l}, \quad \widetilde{\omega}_{l}:=\varphi_{l}^{(1)}-\psi\left(\omega_{l}\right) \in \mathfrak{N}$. 
E. S. VASILEVSKAYA

The formulas $[5,(4.5),(4.13)]$ give invariant representations for the operators $K_{0}$ and $N$ :

$$
\begin{aligned}
K_{0} & =Z S P+S P Z^{*}, \\
N & =Z^{*} X_{1}^{*} R P+(R P)^{*} X_{1} Z .
\end{aligned}
$$

We note that in the basis $\left\{\omega_{l}\right\}_{1}^{n}$ the operator $N_{0}$ is diagonal, whereas the diagonal matrix elements of the operator $N_{*}$ are equal to zero. (Thus, for $n=1$ we have $N_{*}=0$; see [5, Remark 4.3].) Furthermore, formulas (1.4), (1.16) imply

$$
P K_{0} P=0 \text {. }
$$

If $Z=0$, relations (1.11), (1.15), (1.16), and (1.17) show that $F_{1}=0, K_{0}=0, N=0$, and $K=0$.

In [5, (1.8), (4.15), (4.16)] the following estimates were proved:

$$
\begin{aligned}
& \|R\| \leq\left\|X_{1}\right\|, \quad\|S\| \leq\left\|X_{1}\right\|^{2}, \quad\|Z\| \leq(8 \delta)^{-1 / 2}\left\|X_{1}\right\|, \quad\left\|F_{1}\right\| \leq(2 \delta)^{-1 / 2}\left\|X_{1}\right\|, \\
& \left\|K_{0}\right\| \leq(2 \delta)^{-1 / 2}\left\|X_{1}\right\|^{3}, \quad\|N\| \leq(2 \delta)^{-1 / 2}\left\|X_{1}\right\|^{3}, \quad\|K\| \leq 2(2 \delta)^{-1 / 2}\left\|X_{1}\right\|^{3} \\
& \S 2 . \text { Approximation of The operator Exponential } e^{-A(t) \tau}
\end{aligned}
$$

2.1. Approximation of the operator exponential $e^{-A(t) \tau}$. In [15, §2], an approximation for the operator $e^{-A(t) \tau}, \tau \geq 0$, was obtained as $\tau \rightarrow \infty$. It was assumed that

$$
A(t) \geq c_{*} t^{2} I, \quad c_{*}>0, \quad|t| \leq t^{0}
$$

or equivalently,

$$
\lambda_{l}(t) \geq c_{*} t^{2}, \quad l=1, \ldots, n, \quad c_{*}>0, \quad|t| \leq t^{0} .
$$

Here the spectral germ $S$ is nondegenerate, and

$$
S \geq c_{*} I_{\mathfrak{N}}
$$

Theorem 2.1 in [15] gives an approximation for the operator exponential under condition (2.1):

$$
\left\|e^{-A(t) \tau}-P e^{-t^{2} S P \tau}\right\|_{\mathfrak{H} \rightarrow \mathfrak{H}} \leq C_{4}(1+\tau)^{-1 / 2}, \quad \tau \geq 0, \quad|t| \leq t^{0} .
$$

Here

$$
C_{4}:=\sqrt{2} \max \left\{2, C_{3}+\delta^{-1 / 2}\right\}, \quad C_{3}:=\max _{\alpha \geq 0}\left(2 C_{1} \alpha+C_{2} \alpha^{3}\right) e^{-c_{*} \alpha^{2}} .
$$

2.2. Approximation with corrector for the operator $e^{-A(t) \tau}$. Assuming condition (2.1), we rewrite the operator $e^{-A(t) \tau}$ as

$$
e^{-A(t) \tau}=e^{-A(t) \tau} F(t)^{\perp}+e^{-A(t) \tau} F(t) .
$$

Let $\tau>0$. Clearly, for the first term on the right-hand side of (2.4) we have

$$
\left\|e^{-A(t) \tau} F(t)^{\perp}\right\| \leq e^{-\delta \tau} \leq(\delta \tau)^{-1} .
$$

We write the second term in $(2.4)$ as

$$
e^{-A(t) \tau} F(t)=P e^{-A(t) \tau} F(t)+P^{\perp} e^{-A(t) \tau} F(t) .
$$

Consider the second term on the right-hand side of (2.6), taking (1.8) and (1.9) into account:

$$
\begin{aligned}
P^{\perp} e^{-A(t) \tau} F(t) & =(I-P) F(t) e^{-A(t) \tau}=(F(t)-P) F(t) e^{-A(t) \tau}=\Phi(t) F(t) e^{-A(t) \tau} \\
& =\left(t F_{1}+F_{2}(t)\right)\left(\left(e^{-A(t) \tau} F(t)-P e^{-t^{2} S P \tau}\right)+P e^{-t^{2} S P \tau}\right)
\end{aligned}
$$


We denote

$$
e^{-A(t) \tau} F(t)-P e^{-t^{2} S P \tau}=: \Pi(t, \tau)
$$

Then

$$
P^{\perp} e^{-A(t) \tau} F(t)=t F_{1} P e^{-t^{2} S P \tau}+t F_{1} \Pi(t, \tau)+F_{2}(t) e^{-A(t) \tau} F(t) .
$$

We estimate the second term on the right in (2.7). In [15, (2.11)] it was shown that

$$
\|\Pi(t, \tau)\| \leq\left(2 C_{1}|t|+C_{2}|t|^{3} \tau\right) e^{-c_{*} t^{2} \tau} .
$$

Recalling (1.19), we obtain

$$
\left\|t F_{1} \Pi(t, \tau)\right\| \leq(2 \delta)^{-1 / 2}\left\|X_{1}\right\|\left(2 C_{1} t^{2}+C_{2} t^{4} \tau\right) e^{-c_{*} t^{2} \tau} .
$$

Introducing $\alpha:=t^{2} \tau$, we arrive at the estimate

$$
\left\|t F_{1} \Pi(t, \tau)\right\| \leq(2 \delta)^{-1 / 2}\left\|X_{1}\right\|\left(2 C_{1} \alpha+C_{2} \alpha^{2}\right) e^{-c_{*} \alpha} \tau^{-1} .
$$

Now we estimate the third term on the right in (2.7). We use (2.1), (1.10) and denote $\beta^{(2)} \delta^{-1}\left\|X_{1}\right\|^{2}=: C_{5}$. Then

$$
\left\|F_{2}(t) e^{-A(t) \tau} F(t)\right\| \leq C_{5} t^{2} e^{-c_{*} t^{2} \tau}=C_{5} \alpha e^{-c_{*} \alpha} \tau^{-1} .
$$

Combining (2.7), (2.9), and (2.10), we obtain

$$
\left\|P^{\perp} e^{-A(t) \tau} F(t)-t F_{1} P e^{-t^{2} S P \tau}\right\| \leq \varphi(\alpha) \tau^{-1},
$$

where

$$
\varphi(\alpha):=\left(\left(2 C_{1}(2 \delta)^{-1 / 2}\left\|X_{1}\right\|+C_{5}\right) \alpha+C_{2}(2 \delta)^{-1 / 2}\left\|X_{1}\right\| \alpha^{2}\right) e^{-c_{*} \alpha} .
$$

Now we analyze the first term on the right in (2.6):

$$
P e^{-A(t) \tau} F(t)=P e^{-t^{2} S P \tau}+\Sigma(t, \tau),
$$

where

$$
\Sigma(t, \tau):=P e^{-A(t) \tau} F(t)-P e^{-t^{2} S P \tau} .
$$

In [15, §2] the following identity was obtained:

$$
\Sigma(t, \tau)=e^{-t^{2} S P \tau} P \Phi(t)-\int_{0}^{\tau} e^{-t^{2} S P(\tau-\widetilde{\tau})} P \Psi(t) e^{-A(t) \widetilde{\tau}} F(t) d \widetilde{\tau},
$$

where the operators $\Phi(t)$ and $\Psi(t)$ are as in (1.8), (1.12). Using (1.8) and (1.9), we rewrite $\Sigma(t, \tau)$ as

$$
\Sigma(t, \tau)=e^{-t^{2} S P \tau} P F_{1} t+e^{-t^{2} S P \tau} P F_{2}(t)-\int_{0}^{\tau} e^{-t^{2} S P(\tau-\widetilde{\tau})} P \Psi(t) e^{-A(t) \tilde{\tau}} F(t) d \widetilde{\tau}
$$

We estimate the second term on the right in (2.13), taking (2.2) and (1.10) into account:

$$
\left\|e^{-t^{2} S P \tau} P F_{2}(t)\right\| \leq\left\|F_{2}(t)\right\| e^{-c_{*} t^{2} \tau} \leq C_{5} t^{2} e^{-c_{*} t^{2} \tau}=C_{5} \alpha e^{-c_{*} \alpha} \tau^{-1} .
$$

By (1.12) and (1.13), we can transform the third term on the right in (2.13) as follows:

$$
\begin{aligned}
& \int_{0}^{\tau} e^{-t^{2} S P(\tau-\widetilde{\tau})} P \Psi(t) e^{-A(t) \widetilde{\tau}} F(t) d \widetilde{\tau} \\
& =\int_{0}^{\tau} e^{-t^{2} S P(\tau-\widetilde{\tau})} P\left(t^{3} K+\widetilde{\Psi}(t)\right)\left(\Pi(t, \widetilde{\tau})+P e^{-t^{2} S P \widetilde{\tau}}\right) d \widetilde{\tau} \\
& =t^{3} \int_{0}^{\tau} e^{-t^{2} S P(\tau-\widetilde{\tau})} P K P e^{-t^{2} S P \widetilde{\tau}} d \widetilde{\tau}+t^{3} \int_{0}^{\tau} e^{-t^{2} S P(\tau-\widetilde{\tau})} P K \Pi(t, \widetilde{\tau}) d \widetilde{\tau} \\
& +\int_{0}^{\tau} e^{-t^{2} S P(\tau-\widetilde{\tau})} P \widetilde{\Psi}(t) e^{-A(t) \tilde{\tau}} F(t) d \widetilde{\tau} .
\end{aligned}
$$


To estimate the second term on the right in (2.15) we employ (1.20), (2.2), (2.8):

$$
\begin{aligned}
& \left\|t^{3} \int_{0}^{\tau} e^{-t^{2} S P(\tau-\widetilde{\tau})} P K \Pi(t, \widetilde{\tau}) d \widetilde{\tau}\right\| \leq|t|^{3}\|K\| \int_{0}^{\tau} \| e^{-t^{2} S P(\tau-\widetilde{\tau})\|\| \Pi(t, \widetilde{\tau}) \| d \widetilde{\tau}} \\
& \quad \leq|t|^{3}\|K\| \int_{0}^{\tau} e^{-c_{*} t^{2}(\tau-\widetilde{\tau})}\left(2 C_{1}|t|+C_{2}|t|^{3} \widetilde{\tau}\right) e^{-c_{*} t^{2} \widetilde{\tau}} d \widetilde{\tau} \\
& \quad=|t|^{3}\|K\| e^{-c_{*} t^{2} \tau} \int_{0}^{\tau}\left(2 C_{1}|t|+C_{2}|t|^{3} \widetilde{\tau}\right) d \widetilde{\tau}=|t|^{3}\|K\| e^{-c_{*} t^{2} \tau}\left(2 C_{1}|t| \tau+C_{2}|t|^{3} \frac{\tau^{2}}{2}\right) \\
& \quad \leq 2(2 \delta)^{-1 / 2}\left\|X_{1}\right\|^{3}\left(2 C_{1} t^{4} \tau+C_{2} t^{6} \frac{\tau^{2}}{2}\right) e^{-c_{*} t^{2} \tau} .
\end{aligned}
$$

As a result, we arrive at

$$
\left\|t^{3} \int_{0}^{\tau} e^{-t^{2} S P(\tau-\widetilde{\tau})} P K \Pi(t, \widetilde{\tau}) d \widetilde{\tau}\right\| \leq 2(2 \delta)^{-1 / 2}\left\|X_{1}\right\|^{3}\left(2 C_{1} \alpha^{2}+C_{2} \frac{\alpha^{3}}{2}\right) e^{-c_{*} \alpha} \tau^{-1} .
$$

Now we estimate the third term on the right in (2.15) by using (2.1), (2.2), (1.14) and introducing the notation $C_{6}:=\beta \delta^{-1}\left\|X_{1}\right\|^{4}$ :

$$
\begin{gathered}
\left\|\int_{0}^{\tau} e^{-t^{2} S P(\tau-\widetilde{\tau})} P \widetilde{\Psi}(t) e^{-A(t) \tilde{\tau}} F(t) d \widetilde{\tau}\right\| \leq\|\widetilde{\Psi}(t)\| \int_{0}^{\tau}\left\|e^{-t^{2} S P(\tau-\widetilde{\tau})}\right\| \cdot\left\|e^{-A(t) \tilde{\tau}}\right\| d \widetilde{\tau} \\
\leq C_{6} t^{4} \int_{0}^{\tau} e^{-c_{*} t^{2}(\tau-\widetilde{\tau})} e^{-c_{*} t^{2} \widetilde{\tau}} d \widetilde{\tau}=C_{6} t^{4} \tau e^{-c_{*} t^{2} \tau}=C_{6} \alpha^{2} e^{-c_{*} \alpha} \tau^{-1} .
\end{gathered}
$$

Summarizing, we see that (2.12)-(2.17) imply the following estimate:

$$
\begin{gathered}
\left\|P e^{-A(t) \tau} F(t)-\left(P e^{-t^{2} S P \tau}+t e^{-t^{2} S P \tau} P F_{1}-t^{3} \int_{0}^{\tau} e^{-t^{2} S P(\tau-\widetilde{\tau})} P K P e^{-t^{2} S P \tilde{\tau}} d \tilde{\tau}\right)\right\| \\
\leq\left(C_{5} \alpha+\left(C_{6}+4(2 \delta)^{-1 / 2}\left\|X_{1}\right\|^{3} C_{1}\right) \alpha^{2}+C_{2}(2 \delta)^{-1 / 2}\left\|X_{1}\right\|^{3} \alpha^{3}\right) e^{-c_{*} \alpha} \tau^{-1}
\end{gathered}
$$

We use the relations $F_{1} P=Z P$ and $P F_{1}=P Z^{*}$ (see (1.4), (1.11)), and also (1.15), (1.18). Denoting

$$
\mathcal{M}(t, \tau):=\int_{0}^{\tau} e^{-t^{2} S P(\tau-\widetilde{\tau})} P N P e^{-t^{2} S P \widetilde{\tau}} d \widetilde{\tau},
$$

from (2.4)-(2.6), (2.11), and (2.18) we finally obtain

$$
\begin{gathered}
e^{-A(t) \tau}=P e^{-t^{2} S P \tau}+t\left(Z P e^{-t^{2} S P \tau}+e^{-t^{2} S P \tau} P Z^{*}\right)-t^{3} \mathcal{M}(t, \tau)+\mathcal{Y}(t, \tau), \\
\|\mathcal{Y}(t, \tau)\| \leq D_{0} \tau^{-1}, \quad|t| \leq t^{0}, \quad \tau>0
\end{gathered}
$$

Here

$$
\begin{aligned}
D_{0}:= & \max _{\alpha \geq 0}\left(\widetilde{\varphi}(\alpha) e^{-c_{*} \alpha}\right)+\delta^{-1} \\
\widetilde{\varphi}(\alpha):= & \left(2 C_{1}(2 \delta)^{-1 / 2}\left\|X_{1}\right\|+2 C_{5}\right) \alpha \\
& +\left(C_{2}(2 \delta)^{-1 / 2}\left\|X_{1}\right\|+4 C_{1}(2 \delta)^{-1 / 2}\left\|X_{1}\right\|^{3}+C_{6}\right) \alpha^{2} \\
& +C_{2}(2 \delta)^{-1 / 2}\left\|X_{1}\right\|^{3} \alpha^{3} .
\end{aligned}
$$

It is easily seen that the operator $\mathcal{M}(t, \tau)$ is selfadjoint.

Obviously, for $\tau \geq 1$ we have $\tau^{-1} \leq 2(\tau+1)^{-1}$, so that $\|\mathcal{Y}(t, \tau)\| \leq 2 D_{0}(\tau+1)^{-1}$. For $0 \leq \tau<1$ we have $|t|^{3}\|\mathcal{M}(t, \tau)\| \leq 2^{-1 / 2} \delta$ by (1.1), (1.20). Using (1.19) and (2.20), we 
obtain

$$
\|\mathcal{Y}(t, \tau)\| \leq \frac{4+\sqrt{2}}{2}+2^{-1 / 2} \delta<(4+\sqrt{2}(1+\delta))(\tau+1)^{-1}, \quad 0 \leq \tau<1 .
$$

Thus, we arrive at the following assertion.

Theorem 2.1. If $|t| \leq t^{0}$ and $\tau \geq 0$, then

$$
e^{-A(t) \tau}=P e^{-t^{2} S P \tau}+t\left(Z P e^{-t^{2} S P \tau}+e^{-t^{2} S P \tau} P Z^{*}\right)-t^{3} \mathcal{M}(t, \tau)+\mathcal{Y}(t, \tau)
$$

where the operator $\mathcal{M}(t, \tau)$ is defined as in $(2.19)$, and for the operator $\mathcal{Y}(t, \tau)$ we have

$$
\begin{array}{cc}
\|\mathcal{Y}(t, \tau)\| \leq D_{1}(\tau+1)^{-1}, & \tau \geq 0, \\
\|\mathcal{Y}(t, \tau)\| \leq D_{0} \tau^{-1}, & \tau>0, \\
D_{1}:=\max \left\{2 D_{0}, 4+\sqrt{2}(1+\delta)\right\},
\end{array}
$$

with $D_{0}$ defined as in (2.21).

Remark 2.2. In what follows we shall formulate two versions of theorems: for $\tau \geq 0$ and for $\tau>0$. Estimates for $\tau \geq 0$ will be used in the consideration of the nonhomogeneous Cauchy problem (see $\S 10$ ), where we need to integrate in $\tau$ from 0 to $\infty$. On the other hand, estimates for $\tau>0$ enable us to eliminate the smoothing operator $\Pi$ (see Subsection 7.2) from each term of the corrector.

2.3. Calculation of matrix elements of the operator $\mathcal{M}(t, \tau)$. Now we consider the operator $\mathcal{M}(t, \tau)$ in more detail. From (1.15) and (2.19) it follows that

$$
\begin{aligned}
\mathcal{M}(t, \tau) & =\mathcal{M}_{0}(t, \tau)+\mathcal{M}_{*}(t, \tau), \\
\mathcal{M}_{0}(t, \tau) & :=\int_{0}^{\tau} e^{-t^{2} S P(\tau-\widetilde{\tau})} P N_{0} P e^{-t^{2} S P \widetilde{\tau}} d \widetilde{\tau}, \\
\mathcal{M}_{*}(t, \tau) & :=\int_{0}^{\tau} e^{-t^{2} S P(\tau-\widetilde{\tau})} P N_{*} P e^{-t^{2} S P \widetilde{\tau}} d \widetilde{\tau} .
\end{aligned}
$$

Since the operators $S$ and $N_{0}$ have diagonal form in the basis $\left\{\omega_{l}\right\}_{1}^{n}$, we have $N_{0} S=S N_{0}$. Thus,

$$
\mathcal{M}_{0}(t, \tau)=N_{0} e^{-t^{2} S P \tau} P \tau .
$$

In the case where $N=0$ or $N_{*}=0$, formula (2.22) simplifies.

1) Suppose $N=0$. Then

$$
e^{-A(t) \tau}=P e^{-t^{2} S P \tau}+t\left(Z P e^{-t^{2} S P \tau}+e^{-t^{2} S P \tau} P Z^{*}\right)+\mathcal{Y}(t, \tau) .
$$

2) Suppose $N_{*}=0$. Then

$$
e^{-A(t) \tau}=P e^{-t^{2} S P \tau}+t\left(Z P e^{-t^{2} S P \tau}+e^{-t^{2} S P \tau} P Z^{*}\right)-t^{3} N_{0} e^{-t^{2} S P \tau} P \tau+\mathcal{Y}(t, \tau) .
$$

3) Now, we consider the general case. We calculate the matrix elements of the operator $\mathcal{M}_{*}(t, \tau)$ in the basis $\left\{\omega_{l}\right\}_{1}^{n}$. The formula [5, (4.6)] yields

$$
\left(N_{*} \omega_{j}, \omega_{k}\right)=\gamma_{k}\left(\omega_{j}, \widetilde{\omega}_{k}\right)+\gamma_{j}\left(\widetilde{\omega}_{j}, \omega_{k}\right), \quad j, k=1, \ldots, n .
$$

Recall that $\widetilde{\omega}_{j}=\varphi_{j}^{(1)}-\psi\left(\omega_{j}\right)$. We take formula (1.18) in [5] into account:

$$
\left(\widetilde{\omega}_{j}, \omega_{k}\right)=-\left(\omega_{j}, \widetilde{\omega}_{k}\right), \quad j, k=1, \ldots, n .
$$

Then

$$
\left(N_{*} \omega_{j}, \omega_{k}\right)=\left(\gamma_{k}-\gamma_{j}\right)\left(\omega_{j}, \widetilde{\omega}_{k}\right)
$$


Since $S \omega_{l}=\gamma_{l} \omega_{l}$, we obtain

$$
e^{-t^{2} S P \tau} \omega_{j}=e^{-t^{2} \gamma_{j} \tau} \omega_{j} .
$$

The matrix elements of the operator $\mathcal{M}_{*}(t, \tau)$ have the form

$$
\begin{aligned}
\left(\mathcal{M}_{*}(t, \tau) \omega_{j}, \omega_{k}\right) & =\int_{0}^{\tau}\left(e^{-t^{2} S P(\tau-\widetilde{\tau})} N_{*} e^{-t^{2} S P \widetilde{\tau}} \omega_{j}, \omega_{k}\right) d \widetilde{\tau} \\
& =\int_{0}^{\tau}\left(N_{*} e^{-t^{2} \gamma_{j} \widetilde{\tau}} \omega_{j}, e^{-t^{2} \gamma_{k}(\tau-\widetilde{\tau})} \omega_{k}\right) d \widetilde{\tau}
\end{aligned}
$$

or

$$
\left(\mathcal{M}_{*}(t, \tau) \omega_{j}, \omega_{k}\right)=\left(N_{*} \omega_{j}, \omega_{k}\right) e^{-t^{2} \gamma_{k} \tau} \int_{0}^{\tau} e^{t^{2}\left(\gamma_{k}-\gamma_{j}\right) \tau} d \widetilde{\tau} .
$$

Suppose that $\gamma_{k} \neq \gamma_{j}$. Then, by (2.27) and (2.28), we have

$$
\begin{aligned}
\left(\mathcal{M}_{*}(t, \tau) \omega_{j}, \omega_{k}\right) & =\left(N_{*} \omega_{j}, \omega_{k}\right) e^{-t^{2} \gamma_{k} \tau} \frac{e^{t^{2}\left(\gamma_{k}-\gamma_{j}\right) \tau}-1}{\left(\gamma_{k}-\gamma_{j}\right) t^{2}}=\frac{e^{-t^{2} \gamma_{j} \tau}-e^{-t^{2} \gamma_{k} \tau}}{t^{2}}\left(\omega_{j}, \widetilde{\omega}_{k}\right) \\
& =\frac{e^{-t^{2} \gamma_{j} \tau}}{t^{2}}\left(\omega_{j}, \widetilde{\omega}_{k}\right)+\frac{e^{-t^{2} \gamma_{k} \tau}}{t^{2}}\left(\widetilde{\omega}_{j}, \omega_{k}\right)
\end{aligned}
$$

or

$$
\left(\mathcal{M}_{*}(t, \tau) \omega_{j}, \omega_{k}\right)=\frac{1}{t^{2}}\left(\left(e^{-t^{2} \gamma_{j} \tau} \omega_{j}, \widetilde{\omega}_{k}\right)+\left(\widetilde{\omega}_{j}, e^{-t^{2} \gamma_{k} \tau} \omega_{k}\right)\right) .
$$

Relations (2.28) and (2.29) show that for $\gamma_{k}=\gamma_{j}$ we have $\left(\mathcal{M}_{*}(t, \tau) \omega_{j}, \omega_{k}\right)=0$. Thus, the right-hand side of (2.30) is equal to zero by (2.27). Therefore, formula (2.30) is valid also in this case. Using (2.30) and (2.27), we see that

$$
\mathcal{M}_{*}(t, \tau)=-\frac{1}{t^{2}} \sum_{l=1}^{n} e^{-t^{2} \gamma_{l} \tau}\left(\left(\cdot, \tilde{\omega}_{l}\right) \omega_{l}+\left(\cdot, \omega_{l}\right) \tilde{\omega}_{l}\right) .
$$

Finally, (2.25), (2.26), and (2.31) imply that

$$
\mathcal{M}(t, \tau)=N_{0} e^{-t^{2} S P \tau} P \tau-\frac{1}{t^{2}} \sum_{l=1}^{n} e^{-t^{2} \gamma_{l} \tau}\left(\left(\cdot, \widetilde{\omega}_{l}\right) \omega_{l}+\left(\cdot, \omega_{l}\right) \widetilde{\omega}_{l}\right) .
$$

§3. Approximation of the "Bordered" operator exponential $M e^{-A(t) \tau} M^{*}$

3.1. The operator family $A(t)=M^{*} \widehat{A}(t) M$. Following the lines of [4, Subsection 1.5] and [15, Subsection 1.3], we introduce yet another Hilbert space $\hat{\mathfrak{H}}$ and an operator family $\hat{X}(t)=\hat{X}_{0}+t \hat{X}_{1}: \widehat{\mathfrak{H}} \rightarrow \mathfrak{H}_{*}$ that enjoys the assumptions of Subsection 1.1. Let $M: \mathfrak{H} \rightarrow \widehat{\mathfrak{H}}$ be an isomorphism such that $M \operatorname{Dom} X_{0}=\operatorname{Dom} \hat{X}_{0}, X(t)=\hat{X}(t) M: \mathfrak{H} \rightarrow$ $\mathfrak{H}_{*} ; X_{0}=\widehat{X}_{0} M, X_{1}=\hat{X}_{1} M$. Here $A(t)=M^{*} \hat{A}(t) M$, where $\hat{A}(t)=\hat{X}(t)^{*} \hat{X}(t)$. All the objects corresponding to the family $\hat{A}(t)$ will be supplied with the upper mark "^^". Note that $\hat{\mathfrak{N}}=M \mathfrak{N}, \hat{n}=n, \hat{\mathfrak{N}}_{*}=\mathfrak{N}_{*}, \widehat{n}_{*}=n_{*}$, and $\widehat{P}_{*}=P_{*}$. As in [15, (1.10), (1.11), (2.17)], we introduce the notation

$$
Q:=\left(M M^{*}\right)^{-1}: \widehat{\mathfrak{H}} \rightarrow \widehat{\mathfrak{H}}, \quad Q_{\widehat{\mathfrak{N}}}:=\left.\widehat{P} Q\right|_{\hat{\mathfrak{N}}}: \hat{\mathfrak{N}} \rightarrow \widehat{\mathfrak{N}}, \quad M_{0}:=\left(Q_{\widehat{\mathfrak{N}}}\right)^{-1 / 2}
$$

Here $\widehat{P}$ is the orthogonal projection of $\widehat{\mathfrak{H}}$ onto $\hat{\mathfrak{N}}=\operatorname{Ker} \widehat{X}_{0}$. In [15, Proposition 1.2], the following relation was established:

$$
P=M^{-1}\left(Q_{\widehat{\mathfrak{N}}}\right)^{-1} \widehat{P}\left(M^{*}\right)^{-1} .
$$


Our goal in this section is to find an approximation for the operator exponential $e^{-A(t) \tau}$ in terms of the characteristics of $\hat{A}(t)$ and $M$. It turns out to be convenient to approximate the "bordered" exponential $M e^{-A(t) \tau} M^{*}$.

3.2. Approximation of the "bordered" operator exponential $M e^{-A(t) \tau} M^{*}$. By Theorem 2.4 in [15], we have

$$
\left\|M e^{-A(t) \tau} M^{*}-M_{0} e^{-t^{2} M_{0} \widehat{S} M_{0} \tau} M_{0} \widehat{P}\right\|_{\hat{\mathfrak{H}} \rightarrow \hat{\mathfrak{H}}} \leq C_{4}\|M\|^{2}(1+\tau)^{-1 / 2}, \quad \tau \geq 0, \quad|t| \leq t^{0},
$$

where $\widehat{S}$ is the spectral germ of the operator $\widehat{A}(t)$ and $C_{4}$ is the constant in (2.3). We want to obtain an approximation with corrector for the operator $M e^{-A(t) \tau} M^{*}$. We use Theorem 2.1. From (2.22) it follows that, for $\tau \geq 0$ and $|t| \leq t^{0}$, we have

$$
\begin{aligned}
M e^{-A(t) \tau} M^{*}= & M e^{-t^{2} S P \tau} P M^{*}+t\left(M Z e^{-t^{2} S P \tau} P M^{*}+M e^{-t^{2} S P \tau} P Z^{*} M^{*}\right) \\
& -t^{3} \int_{0}^{\tau} M e^{-t^{2} S P(\tau-\widetilde{\tau})} P N P e^{-t^{2} S P \widetilde{\tau}} P M^{*} d \widetilde{\tau}+M \mathcal{Y}(t, \tau) M^{*}
\end{aligned}
$$

We use [15, (2.19)]:

$$
M e^{-t^{2} S P \tau} P M^{*}=M_{0} e^{-t^{2} M_{0} \hat{S} M_{0} \tau} M_{0} \widehat{P} .
$$

Also, we use the notation introduced in [5, (6.16), (6.18)]:

$$
\widehat{Z}_{Q}:=M Z M^{-1} \widehat{P}, \quad \widehat{N}_{Q}:=\widehat{P}\left(M^{*}\right)^{-1} N M^{-1} \widehat{P} .
$$

Then (3.2) and estimates (2.23) imply the following result.

Theorem 3.1. For $|t| \leq t^{0}$ we have

$$
\begin{aligned}
M e^{-A(t) \tau} M^{*}= & M_{0} e^{-t^{2} M_{0} \hat{S} M_{0} \tau} M_{0} \widehat{P} \\
& +t\left(\hat{Z}_{Q} M_{0} e^{-t^{2} M_{0} \hat{S} M_{0} \tau} M_{0} \widehat{P}+M_{0} e^{-t^{2} M_{0} \hat{S} M_{0} \tau} M_{0} \widehat{P} \widehat{Z}_{Q}^{*}\right) \\
& -t^{3} \int_{0}^{\tau} M_{0} e^{-t^{2} M_{0} \hat{S} M_{0}(\tau-\widetilde{\tau})} M_{0} \widehat{N}_{Q} M_{0} e^{-t^{2} M_{0} \hat{S} M_{0} \tilde{\tau}} M_{0} \widehat{P} d \widetilde{\tau}+\mathcal{Y}_{M}(t, \tau),
\end{aligned}
$$

where

$$
\begin{aligned}
\left\|\mathcal{Y}_{M}(t, \tau)\right\| & \leq\|M\|^{2} D_{1}(\tau+1)^{-1}, & & \tau \geq 0, \\
\left\|\mathcal{Y}_{M}(t, \tau)\right\| & \leq\|M\|^{2} D_{0} \tau^{-1}, & & \tau>0,
\end{aligned}
$$

and $D_{0}, D_{1}$ are the constants defined in (2.21), (2.24).

Now we give expressions for the operators $\hat{Z}_{Q}$ and $\hat{N}_{Q}$ similar to (1.3), (1.17). In Lemma 6.1 in [5], it was shown that $\widehat{Z}_{Q}$ is the operator in $\widehat{\mathfrak{H}}$ that takes an element $\widehat{u} \in \hat{\mathfrak{H}}$ to a unique solution $\widehat{\psi}_{Q} \in \hat{\mathfrak{H}}$ of the equation

$$
\widehat{X}_{0}^{*}\left(\hat{X}_{0} \widehat{\psi}_{Q}+\hat{X}_{1} \widehat{\omega}\right)=0, \quad Q \widehat{\psi}_{Q} \perp \hat{\mathfrak{N}}
$$

where $\widehat{\omega}=\widehat{P} \widehat{u} \in \hat{\mathfrak{N}}$.

An expression for the operator $\hat{N}_{Q}$ was obtained in Lemma 6.2 of [5]:

$$
\widehat{N}_{Q}=\widehat{Z}_{Q}^{*} \hat{X}_{1}^{*} \widehat{R} \hat{P}+(\widehat{R} \hat{P})^{*} \hat{X}_{1} \widehat{Z}_{Q}
$$




\section{Chapter 2. PERIODIC DIFFERENTIAL OPERATORS}

\section{$\S 4$. Description of a Class of operators}

The material of $\S 4$ is borrowed from [4].

4.1. Factorized second-order operators. We consider selfadjoint matrix secondorder differential operators (DO's) of a special form that was described in detail in [4, Subsection 2.1.1].

We put $\mathbf{D}:=-i\left(\partial / \partial x_{1}, \ldots, \partial / \partial x_{d}\right)$, and let $b(\mathbf{D}): L_{2}\left(\mathbb{R}^{d} ; \mathbb{C}^{n}\right) \rightarrow L_{2}\left(\mathbb{R}^{d} ; \mathbb{C}^{m}\right)$ be a homogeneous first-order DO with constant coefficients. Suppose that $m \geq n$. In fixed orthonormal bases $\widetilde{\mathbf{e}}_{1}, \ldots, \widetilde{\mathbf{e}}_{n}$ in $\mathbb{C}^{n}, \mathbf{e}_{1}, \ldots, \mathbf{e}_{m}$ in $\mathbb{C}^{m}$, and $\mathbf{e}_{1}^{\prime}, \ldots, \mathbf{e}_{d}^{\prime}$ in $\mathbb{R}^{d}$, the operator $b(\mathbf{D})$ is associated with an $(m \times n)$-matrix $b(\boldsymbol{\xi})$, which is called the symbol of $b(\mathbf{D})$. The matrix $b(\boldsymbol{\xi})$ is a linear homogeneous function of $\boldsymbol{\xi} \in \mathbb{R}^{d}$. We assume that

$$
\operatorname{rank} b(\boldsymbol{\xi})=n, \quad 0 \neq \boldsymbol{\xi} \in \mathbb{R}^{d},
$$

or equivalently,

$$
\alpha_{0} \mathbf{1}_{n} \leq b(\boldsymbol{\theta})^{*} b(\boldsymbol{\theta}) \leq \alpha_{1} \mathbf{1}_{n}, \quad|\boldsymbol{\theta}|=1, \quad 0<\alpha_{0} \leq \alpha_{1}<\infty .
$$

On the domain

$$
\operatorname{Dom} \mathcal{X}:=\left\{\mathbf{u} \in L_{2}\left(\mathbb{R}^{d} ; \mathbb{C}^{n}\right): f \mathbf{u} \in H^{1}\left(\mathbb{R}^{d} ; \mathbb{C}^{n}\right)\right\},
$$

we consider a closed operator

$$
\mathcal{X}:=h b(\mathbf{D}) f: L_{2}\left(\mathbb{R}^{d} ; \mathbb{C}^{n}\right) \rightarrow L_{2}\left(\mathbb{R}^{d} ; \mathbb{C}^{m}\right) .
$$

Here $h(\mathbf{x})$ for $\mathbf{x} \in \mathbb{R}^{d}$ is an $(m \times m)$-matrix-valued function, $f(\mathbf{x})$ for $\mathbf{x} \in \mathbb{R}^{d}$ is an $(n \times n)$-matrix-valued function, and

$$
h, h^{-1} \in L_{\infty}\left(\mathbb{R}^{d}\right), \quad f, f^{-1} \in L_{\infty}\left(\mathbb{R}^{d}\right) .
$$

In $L_{2}\left(\mathbb{R}^{d} ; \mathbb{C}^{n}\right)$, we introduce the selfadjoint operator

$$
\mathcal{A}:=\mathcal{X}^{*} \mathcal{X}
$$

The operator $\mathcal{A}$ is generated by the closed quadratic form

$$
a[\mathbf{u}, \mathbf{u}]:=\|\mathcal{X} \mathbf{u}\|_{L_{2}\left(\mathbb{R}^{d} ; \mathbb{C}^{m}\right)}^{2}, \quad u \in \operatorname{Dom} \mathcal{X} .
$$

Let

$$
g(\mathbf{x}):=h(\mathbf{x})^{*} h(\mathbf{x})
$$

Then, formally,

$$
\mathcal{A}=f(\mathbf{x})^{*} b(\mathbf{D})^{*} g(\mathbf{x}) b(\mathbf{D}) f(\mathbf{x}) .
$$

Note that the following estimates are valid (see [4, (2.1.11)]):

$$
\begin{gathered}
c_{0} \int_{\mathbb{R}^{d}}|\mathbf{D}(f \mathbf{u})|^{2} d \mathbf{x} \leq a[\mathbf{u}, \mathbf{u}] \leq c_{1} \int_{\mathbb{R}^{d}}|\mathbf{D}(f \mathbf{u})|^{2} d \mathbf{x}, \quad \mathbf{u} \in \operatorname{Dom} \mathcal{X} \\
c_{0}=\alpha_{0}\left\|g^{-1}\right\|_{L_{\infty}}^{-1}, \quad c_{1}=\alpha_{1}\|g\|_{L_{\infty}} .
\end{gathered}
$$


4.2. The Gelfand transformation. Let $\Gamma$ be a lattice in $\mathbb{R}^{d}$, i.e.,

$$
\Gamma=\left\{\mathbf{a} \in \mathbb{R}^{d}: \mathbf{a}=\sum_{i=1}^{d} n_{i} \mathbf{a}_{i}, n_{i} \in \mathbb{Z}\right\},
$$

where $\mathbf{a}_{1}, \ldots, \mathbf{a}_{d}$ is a basis in $\mathbb{R}^{d}$. Let $\widetilde{\Gamma}$ be the lattice dual to $\Gamma$ :

$$
\widetilde{\Gamma}=\left\{\mathbf{b} \in \mathbb{R}^{d}: \mathbf{b}=\sum_{i=1}^{d} m_{i} \mathbf{b}_{i}, m_{i} \in \mathbb{Z}\right\},
$$

where $\mathbf{b}_{1}, \ldots, \mathbf{b}_{d}$ is the basis in $\mathbb{R}^{d}$ dual to $\mathbf{a}_{1}, \ldots, \mathbf{a}_{d}:\left\langle\mathbf{b}_{i}, \mathbf{a}_{j}\right\rangle=2 \pi \delta_{i j}$. We denote by $\Omega$ the elementary cell of the lattice $\Gamma$,

$$
\Omega:=\left\{\mathbf{x} \in \mathbb{R}^{d}: \mathbf{x}=\sum_{i=1}^{d} x_{i} \mathbf{a}_{i}, 0<x_{i}<1\right\},
$$

and by $\widetilde{\Omega}$ the Brillouin zone of the dual lattice,

$$
\widetilde{\Omega}:=\left\{\mathbf{k} \in \mathbb{R}^{d}:|\mathbf{k}|<|\mathbf{k}-\mathbf{b}|, 0 \neq \mathbf{b} \in \widetilde{\Gamma}\right\} .
$$

We use the notation $|\Omega|:=$ meas $\Omega,|\widetilde{\Omega}|:=$ meas $\widetilde{\Omega}$. Note that $|\Omega||\widetilde{\Omega}|=(2 \pi)^{d}$.

The Gelfand transformation (see, e.g., 4, §2.1]) is defined initially on the functions of the Schwartz class $\mathcal{S}$ by the formula

$$
\begin{array}{r}
\widetilde{\mathbf{v}}(\mathbf{k}, \mathbf{x}):=(\mathcal{U} \mathbf{v})(\mathbf{k}, \mathbf{x})=|\widetilde{\Omega}|^{-1 / 2} \sum_{\mathbf{a} \in \Gamma} \exp (-i\langle\mathbf{k}, \mathbf{x}+\mathbf{a}\rangle) \mathbf{v}(\mathbf{x}+\mathbf{a}), \\
\mathbf{v} \in \mathcal{S}\left(\mathbb{R}^{d}, \mathbb{C}^{n}\right), \quad \mathbf{x} \in \Omega, \quad \mathbf{k} \in \widetilde{\Omega} .
\end{array}
$$

It is an isometry from $L_{2}\left(\mathbb{R}^{d} ; \mathbb{C}^{n}\right)$ to $L_{2}(\widetilde{\Omega} \times \Omega)$, i.e.,

$$
\int_{\widetilde{\Omega}} \int_{\Omega}|\widetilde{\mathbf{v}}(\mathbf{k}, \mathbf{x})|^{2} d \mathbf{x} d \mathbf{k}=\int_{\mathbb{R}^{d}}|\mathbf{v}(\mathbf{x})|^{2} d \mathbf{x}
$$

Therefore, $\mathcal{U}$ extends from the Schwartz class to a unitary mapping

$$
\mathcal{U}: L_{2}\left(\mathbb{R}^{d} ; \mathbb{C}^{n}\right) \rightarrow \int_{\widetilde{\Omega}} \oplus L_{2}\left(\Omega ; \mathbb{C}^{n}\right) d \mathbf{k}=: \mathcal{H}
$$

It turns out that $\mathbf{v} \in H^{1}\left(\mathbb{R}^{d} ; \mathbb{C}^{n}\right)$ if and only if $\widetilde{\mathbf{v}} \in L_{2}\left(\widetilde{\Omega} ; \widetilde{H}^{1}\left(\Omega ; \mathbb{C}^{n}\right)\right)$, where $\widetilde{H}^{1}\left(\Omega ; \mathbb{C}^{n}\right)$ stands for the subspace of all functions in $H^{1}\left(\Omega ; \mathbb{C}^{n}\right)$ whose $\Gamma$-periodic extensions to $\mathbb{R}^{d}$ belong to $H_{\text {loc }}^{1}\left(\mathbb{R}^{d} ; \mathbb{C}^{n}\right)$. Moreover, under the transformation $\mathcal{U}$, the operator in $L_{2}\left(\mathbb{R}^{d} ; \mathbb{C}^{n}\right)$ of multiplication by a bounded function turns into multiplication by the same function on the fibers of the direct integral $\mathcal{H}$, and the operator $b(\mathbf{D})$ applied to $\mathbf{v} \in H^{1}\left(\mathbb{R}^{d} ; \mathbb{C}^{n}\right)$ turns on these fibers into the operator $b(\mathbf{D}+\mathbf{k})$ applied to $\widetilde{\mathbf{v}}(\mathbf{k}, \cdot) \in$ $\widetilde{H}^{1}\left(\Omega ; \mathbb{C}^{n}\right)$.

4.3. Expansion of $\mathcal{A}$ into a direct integral. By using the remarks in Subsection 4.2, we can diagonalize the operator $\mathcal{A}$ partially in the direct integral $\mathcal{H}$; see 4 , Subsection 2.2.1, 2.2.3]. Consider the closed operator

$$
\mathcal{X}(\mathbf{k}):=h b(\mathbf{D}+\mathbf{k}) f: L_{2}\left(\Omega ; \mathbb{C}^{n}\right) \rightarrow L_{2}\left(\Omega ; \mathbb{C}^{m}\right), \quad \mathbf{k} \in \mathbb{R}^{d},
$$

on the domain

$$
\operatorname{Dom} \mathcal{X}(\mathbf{k}):=\left\{\mathbf{u} \in L_{2}\left(\Omega ; \mathbb{C}^{n}\right): f \mathbf{u} \in \widetilde{H}^{1}\left(\Omega ; \mathbb{C}^{n}\right)\right\} .
$$

We define a selfadjoint operator

$$
\mathcal{A}(\mathbf{k}):=\mathcal{X}(\mathbf{k})^{*} \mathcal{X}(\mathbf{k}): L_{2}\left(\Omega ; \mathbb{C}^{n}\right) \rightarrow L_{2}\left(\Omega ; \mathbb{C}^{n}\right), \quad \mathbf{k} \in \mathbb{R}^{d},
$$


which corresponds to the closed quadratic form

$$
a(\mathbf{k})[\mathbf{u}, \mathbf{u}]:=\|\mathcal{X}(\mathbf{k}) \mathbf{u}\|_{L_{2}\left(\Omega ; \mathbb{C}^{m}\right)}^{2}, \quad \mathbf{u} \in \operatorname{Dom} \mathcal{X}(\mathbf{k}), \quad \mathbf{k} \in \mathbb{R}^{d} .
$$

Note that

$$
c_{0} \int_{\Omega}|(\mathbf{D}+\mathbf{k}) \mathbf{u}|^{2} d \mathbf{x} \leq a(\mathbf{k})[\mathbf{u}, \mathbf{u}] \leq c_{1} \int_{\Omega}|(\mathbf{D}+\mathbf{k}) \mathbf{u}|^{2} d \mathbf{x},
$$

where the constants $c_{0}$ and $c_{1}$ are defined as in (4.5).

Suppose that $\mathbf{u} \in \operatorname{Dom} a$, where the form $a$ is defined in (4.3). We denote $\widetilde{\mathbf{u}}:=$ $\mathcal{U} \mathbf{u}, \mathbf{v}:=f \mathbf{u}$. Then $\mathbf{u} \in \operatorname{Dom} a$ if and only if $\widetilde{\mathbf{u}}(\mathbf{k}, \cdot) \in \operatorname{Dom} \mathcal{X}(\mathbf{k})$ for almost all $\mathbf{k} \in \mathbb{R}^{d}$ and

Here

$$
\int_{\widetilde{\Omega}} a(\mathbf{k})[\widetilde{\mathbf{u}}(\mathbf{k}, \cdot), \widetilde{\mathbf{u}}(\mathbf{k}, \cdot)] d \mathbf{k}<\infty .
$$

$$
a[\mathbf{u}, \mathbf{u}]=\int_{\widetilde{\Omega}} a(\mathbf{k})[\widetilde{\mathbf{u}}(\mathbf{k}, \cdot), \widetilde{\mathbf{u}}(\mathbf{k}, \cdot)] d \mathbf{k} .
$$

This means that the action of the operator $\mathcal{A}(\mathbf{k})$ in the direct integral $\mathcal{H}$ turns into fiberwise multiplication by the operator-valued function $\mathcal{A}(\mathbf{k}), \mathbf{k} \in \widetilde{\Omega}$, which can be written in the following form:

$$
\mathcal{U} \mathcal{A} \mathcal{U}^{-1}=\int_{\tilde{\Omega}} \oplus \mathcal{A}(\mathbf{k}) d \mathbf{k}
$$

The compactness of the embedding of $\widetilde{H}^{1}\left(\Omega ; \mathbb{C}^{n}\right)$ in $L_{2}\left(\Omega ; \mathbb{C}^{n}\right)$ and inequalities (4.8) show that the spectrum of $\mathcal{A}(\mathbf{k})$ is discrete. For the consecutive eigenvalues of the operator $\mathcal{A}(\mathbf{k})$ (with multiplicities), we introduce the notation

$$
E_{1}(\mathbf{k}) \leq E_{2}(\mathbf{k}) \leq \cdots \leq E_{s}(\mathbf{k}) \leq \cdots, \quad \mathbf{k} \in \mathbb{R}^{d} .
$$

The functions $E_{s}(\mathbf{k})$ are called band functions. These functions are continuous and $\widetilde{\Gamma}$-periodic. The spectrum of the operator $\mathcal{A}$ coincides with the union of the segments that are the images of the band functions (4.10):

$$
\operatorname{spec} \mathcal{A}=\bigcup_{j \in \mathbb{N}} \operatorname{Ran} E_{j} .
$$

4.4. Incorporation of the operators $\mathcal{A}(\mathbf{k})$ into the abstract framework. In order to incorporate the operators $\mathcal{A}(\mathbf{k})$ into the abstract framework, a one-dimensional parameter $t=|\mathbf{k}|$ was introduced in [4, Subsection 2.3.1]. We put $\mathbf{k}=t \boldsymbol{\theta},|\boldsymbol{\theta}|=1$. Suppose that $\mathfrak{H}=L_{2}\left(\Omega ; \mathbb{C}^{n}\right), \mathfrak{H}_{*}=L_{2}\left(\Omega ; \mathbb{C}^{m}\right)$. As in [4. Subsection 2.3.1], we put

$$
\begin{aligned}
X(t) & =X(t, \boldsymbol{\theta})=\mathcal{X}(t \boldsymbol{\theta})=h b(\mathbf{D}+t \boldsymbol{\theta}) f, \\
X_{0} & =\mathcal{X}(0)=h b(\mathbf{D}) f, \quad \operatorname{Dom} X_{0}=\left\{\mathbf{u} \in \mathfrak{H}: f \mathbf{u} \in \widetilde{H}^{1}\left(\Omega ; \mathbb{C}^{n}\right)\right\}, \\
X_{1} & =X_{1}(\boldsymbol{\theta})=h b(\boldsymbol{\theta}) f .
\end{aligned}
$$

Then $X(t, \boldsymbol{\theta})=X_{0}+t X_{1}(\boldsymbol{\theta})$. Finally,

$$
A(t)=A(t, \boldsymbol{\theta})=\mathcal{A}(t \boldsymbol{\theta}) .
$$

For the kernel $\operatorname{Ker} \mathcal{A}(0)=\operatorname{Ker} X_{0}$ we have

$$
\mathfrak{N}=\operatorname{Ker} A(0)=\left\{\mathbf{u} \in \mathfrak{H}: f \mathbf{u}=\mathbf{c} \in \mathbb{C}^{n}\right\}, \quad \operatorname{dim} \mathfrak{N}=n .
$$

The assumption $m \geq n$ implies that $n \leq n_{*}$.

As was shown in [4, (2.2.14)], the estimate $E_{n+1}(0) \geq 4 c_{*} r_{0}^{2}$ is valid, where $r_{0}$ is the radius of the ball inscribed in the Brillouin zone $\widetilde{\Omega}$, and

$$
c_{*}:=\alpha_{0}\left\|f^{-1}\right\|_{L_{\infty}}^{-2}\left\|h^{-1}\right\|_{L_{\infty}}^{-2}=\alpha_{0}\left\|f^{-1}\right\|_{L_{\infty}}^{-2}\left\|g^{-1}\right\|_{L_{\infty}}^{-1} .
$$


We see that the distance $d_{0}$ from the point $\lambda=0$ to the rest of the spectrum of the operator $A(0)$ admits the following lower estimate:

$$
d_{0} \geq 4 c_{*} r_{0}^{2} .
$$

In accordance with the assumption $8 \delta<d_{0}$ (see Subsection 1.1), we fix the number $\delta$ :

$$
\delta=c_{*} r_{0}^{2} / 4
$$

Since (4.11) and (4.2) imply that

$$
\left\|X_{1}(\boldsymbol{\theta})\right\| \leq \alpha_{1}^{1 / 2}\|f\|_{L_{\infty}}\|h\|_{L_{\infty}},
$$

we can take $t^{0}$ in (1.1) less than $\delta^{1 / 2}\left\|X_{1}(\boldsymbol{\theta})\right\|^{-1}$ and not depending on $\boldsymbol{\theta}$ :

$$
t^{0}:=\delta^{1 / 2} \alpha_{1}^{-1 / 2}\|f\|_{L_{\infty}}^{-1}\|h\|_{L_{\infty}}^{-1}=\delta^{1 / 2} \alpha_{1}^{-1 / 2}\|f\|_{L_{\infty}}^{-1}\|g\|_{L_{\infty}}^{-1 / 2} .
$$

The required inequalities (2.1), (2.2) follow from [4, (2.2.12)]:

$$
E_{l}(\mathbf{k}) \geq c_{*}|\mathbf{k}|^{2}, \quad l=1, \ldots, n, \quad \mathbf{k} \in \operatorname{clos} \widetilde{\Omega},
$$

where the constant $c_{*}$ is as in (4.13). Therefore, we arrive at the estimates

$$
\lambda_{l}(t, \boldsymbol{\theta}) \geq c_{*} t^{2}, \quad l=1, \ldots, n, \quad t \in\left[0, t^{0}\right],
$$

which imply (2.1) and (2.2). Moreover, the spectral germ $S(\boldsymbol{\theta})$ is nondegenerate:

$$
S(\boldsymbol{\theta}) \geq c_{*} I_{\mathfrak{N}} .
$$

Along with the general operator (4.4), it is convenient to denote the special case of this operator where $f=\mathbf{1}_{n}$ by

$$
\widehat{\mathcal{A}}:=b(\mathbf{D})^{*} g(\mathbf{x}) b(\mathbf{D}) .
$$

Then

$$
\mathcal{A}=f^{*} \widehat{\mathcal{A}} f .
$$

All the objects related to the case where $f=\mathbf{1}_{n}$ will be marked by the upper sign "^^". Let $\widehat{\mathcal{A}}(k)=\widehat{A}(t, \boldsymbol{\theta})$ be the operator family of the form (4.7) for the case where $f=\mathbf{1}_{n}$. Then $\hat{X}(t, \boldsymbol{\theta})=\widehat{X}_{0}+t \hat{X}_{1}(\boldsymbol{\theta})$, where

$$
\hat{X}_{0}=h(\mathbf{x}) b(\mathbf{D}), \quad \hat{X}_{1}(\boldsymbol{\theta})=h(\mathbf{x}) b(\boldsymbol{\theta}),
$$

and the kernel $\hat{\mathfrak{N}}$ (see (4.12)) has the form

$$
\hat{\mathfrak{N}}=\left\{\mathbf{u} \in \mathfrak{H}: \mathbf{u}=\mathbf{c} \in \mathbb{C}^{n}\right\} .
$$

Here the orthogonal projection $\widehat{P}$ onto $\hat{\mathfrak{N}}$ is the operator of averaging over the cell:

$$
\widehat{P} \mathbf{u}=|\Omega|^{-1} \int_{\Omega} \mathbf{u}(\mathbf{x}) d \mathbf{x}, \quad \mathbf{u} \in \mathfrak{H} .
$$

The role of $\widehat{A}(t)$ from $\S 3$ is played by $\widehat{A}(t, \boldsymbol{\theta})=\widehat{\mathcal{A}}(\mathbf{k})$, and the role of the isomorphism $M$ is played by the operator of multiplication by the matrix-valued function $f(\mathbf{x})$ :

$$
A(t, \boldsymbol{\theta})=f^{*} \widehat{A}(t, \boldsymbol{\theta}) f .
$$

The operator $Q$ occurring in (3.1) is now the operator of multiplication by the matrixvalued function

$$
Q(\mathbf{x}):=\left(f(\mathbf{x}) f(\mathbf{x})^{*}\right)^{-1} .
$$

We introduce the following notation. Let $\phi(\mathbf{x})$ be a $\Gamma$-periodic function whose values are nonsingular square matrices, and let $\phi, \phi^{-1} \in L_{1, \text { loc }}\left(\mathbb{R}^{d}\right)$. Then

$$
\bar{\phi}:=|\Omega|^{-1} \int_{\Omega} \phi(\mathbf{x}) d \mathbf{x}, \quad \underline{\phi}:=\left(|\Omega|^{-1} \int_{\Omega} \phi(\mathbf{x})^{-1} d \mathbf{x}\right)^{-1} .
$$


E. S. VASILEVSKAYA

If $\phi(\mathbf{x})>0$ we always have $\phi \leq \bar{\phi}$, with equality only in the case of a constant matrix $\phi$.

Now the operators $Q_{\hat{\mathfrak{N}}}$ and $M_{0}$ (see (3.1)) become the operators of multiplication by constant matrices:

$$
Q_{\widehat{\mathfrak{N}}}=\bar{Q}=\left(\underline{f f^{*}}\right)^{-1}, \quad M_{0}=(\bar{Q})^{-1 / 2}=\left(\underline{f f^{*}}\right)^{1 / 2}=: f_{0} .
$$

\section{§5. EfFective characteristics}

The material of $\S 5$ is borrowed from [4, 5, 6].

5.1. The effective matrix and the effective operator. We apply the method of Chapter 1 to the operator $\mathcal{A}(\mathbf{k})=A(t, \boldsymbol{\theta})$. We have $A(t, \boldsymbol{\theta})=f^{*} \widehat{A}(t, \boldsymbol{\theta}) f$. Now the operators $\widehat{S}, \widehat{Z}_{Q}, \widehat{N}_{Q}$ (see $\S 3$ ) depend on $\boldsymbol{\theta}$.

In [4. Chapter $3, \S 1$ ] it was shown that the spectral germ $\widehat{S}(\boldsymbol{\theta})$ of the family $\widehat{A}(t, \boldsymbol{\theta})=$ $\widehat{\mathcal{A}}(\mathbf{k})$ can be represented as

$$
\widehat{S}(\boldsymbol{\theta})=b(\boldsymbol{\theta})^{*} g^{0} b(\boldsymbol{\theta}),
$$

where $g^{0}$ is some constant matrix. In order to find it, we consider a set of equations in functions $\mathbf{v}_{j} \in \widetilde{H}^{1}\left(\Omega ; \mathbb{C}^{n}\right), j=1, \ldots, m$ :

$$
b(\mathbf{D})^{*} g(\mathbf{x})\left(b(\mathbf{D}) \mathbf{v}_{j}(\mathbf{x})+\mathbf{e}_{j}\right)=0, \quad \int_{\Omega} \mathbf{v}_{j}(\mathbf{x}) d \mathbf{x}=0,
$$

which are understood in the weak sense. The columns $\mathbf{v}_{j}(\mathbf{x})$ form a periodic $(n \times m)$ matrix-valued function $\Lambda(\mathbf{x})$. We denote by $\Lambda$ the operator of multiplication by the matrix $\Lambda(\mathbf{x})$. Next, we introduce the matrix

$$
\widetilde{g}(\mathbf{x}):=g(\mathbf{x})\left(b(\mathbf{D}) \Lambda(\mathbf{x})+\mathbf{1}_{m}\right),
$$

and finally, we define the effective matrix $g^{0}$ :

$$
g^{0}:=|\Omega|^{-1} \int_{\Omega} \tilde{g}(\mathbf{x}) d \mathbf{x} .
$$

Note that the effective matrix $g^{0}$ satisfies the inequalities

$$
\underline{g} \leq g^{0} \leq \bar{g}
$$

(see (4.23)), which is known as the Voigt-Reuss bracketing (see [4, Theorem 3.1.5]).

We denote the operator family $\widehat{\mathcal{A}}(\mathbf{k})=\widehat{A}(t, \boldsymbol{\theta})$ by $\hat{A}(t, \boldsymbol{\theta} ; g)$ and the corresponding germ $\widehat{S}(\boldsymbol{\theta})$ by $\widehat{S}(\boldsymbol{\theta} ; g)$. As was proved in [4, Subsection 3.1.2], the consideration of the family $\widehat{A}\left(t, \boldsymbol{\theta} ; g^{0}\right)$ shows that

$$
\widehat{S}(\boldsymbol{\theta} ; g)=\widehat{S}\left(\boldsymbol{\theta} ; g^{0}\right)
$$

Since the kernel (4.20) is independent of $g$, the operator families $\widehat{A}(t, \boldsymbol{\theta} ; g)$ and $\widehat{A}\left(t, \boldsymbol{\theta} ; g^{0}\right)$ are threshold equivalent (see the definition in Subsection 1.3). We denote

$$
\widehat{S}\left(\mathbf{k} ; g^{0}\right):=t^{2} \widehat{S}\left(\boldsymbol{\theta} ; g^{0}\right)=b(\mathbf{k})^{*} g^{0} b(\mathbf{k}), \quad \mathbf{k}=t \boldsymbol{\theta} .
$$

Here the operator $\widehat{S}\left(\mathbf{k} ; g^{0}\right)$ is the symbol of the DO

$$
\widehat{\mathcal{A}}^{0}:=b(\mathbf{D})^{*} g^{0} b(\mathbf{D})
$$

with constant coefficients, which is called the effective operator for the operator (4.19). 
5.2. The operators $\hat{Z}_{Q}(\boldsymbol{\theta})$ and $\hat{N}_{Q}(\boldsymbol{\theta})$. In accordance with Subsection 3.2 , the operator $\hat{Z}_{Q}(\boldsymbol{\theta})$ applied to an element $\hat{\mathbf{u}} \in L_{2}\left(\Omega ; \mathbb{C}^{n}\right)$ gives a solution $\mathbf{w}^{(Q)} \in \widetilde{H}^{1}\left(\Omega ; \mathbb{C}^{n}\right)$ of the problem

$$
b(\mathbf{D})^{*} g(\mathbf{x})\left(b(\mathbf{D}) \mathbf{w}^{(Q)}(\mathbf{x})+b(\boldsymbol{\theta}) \mathbf{c}\right)=0, \quad \int_{\Omega} Q(\mathbf{x}) \mathbf{w}^{(Q)}(\mathbf{x}) d \mathbf{x}=0, \quad \mathbf{c}=\hat{P} \widehat{\mathbf{u}} .
$$

By analogy with the operator $\Lambda$, we introduce the operator $\Lambda_{Q}$, which is multiplication by the periodic $(n \times m)$-matrix-valued function $\Lambda_{Q}(\mathbf{x})$ with the columns $\mathbf{v}_{j}^{(Q)}(\mathbf{x})$, where the $\mathbf{v}_{j}^{(Q)}(\mathbf{x}) \in \widetilde{H}^{1}\left(\Omega ; \mathbb{C}^{n}\right), j=1, \ldots, m$, are the solutions of the problems

$$
b(\mathbf{D})^{*} g(\mathbf{x})\left(b(\mathbf{D}) \mathbf{v}_{j}^{(Q)}(\mathbf{x})+\mathbf{e}_{j}\right)=0, \quad \int_{\Omega} Q(\mathbf{x}) \mathbf{v}_{j}^{(Q)}(\mathbf{x}) d \mathbf{x}=0 .
$$

We need the following formula [6, (5.4)]:

$$
\Lambda_{Q}(\mathbf{x})=\Lambda(\mathbf{x})+\Lambda_{Q}^{0}, \quad \Lambda_{Q}^{0}=-(\bar{Q})^{-1}(\overline{Q \Lambda}) ;
$$

i.e., the matrix $\Lambda_{Q}(\mathbf{x})$ differs from $\Lambda(\mathbf{x})$ by the constant term $\Lambda_{Q}^{0}$.

The operator $\hat{Z}_{Q}(\boldsymbol{\theta})$ has the form

$$
\widehat{Z}_{Q}(\boldsymbol{\theta})=\Lambda_{Q} b(\boldsymbol{\theta}) \hat{P} .
$$

We put

$$
\widehat{Z}_{Q}(\mathbf{k}):=t \widehat{Z}_{Q}(\boldsymbol{\theta})=\Lambda_{Q} b(\mathbf{k}) \hat{P}, \quad \mathbf{k}=t \boldsymbol{\theta} .
$$

In [6, (5.11), (5.13)] it was shown that the operator $\hat{N}_{Q}(\boldsymbol{\theta})$ looks like this:

$$
\widehat{N}_{Q}(\boldsymbol{\theta})=b(\boldsymbol{\theta})^{*} L_{Q}(\boldsymbol{\theta}) b(\boldsymbol{\theta}) \hat{P},
$$

where $L_{Q}(\boldsymbol{\theta})$ is a Hermitian $(m \times m)$-matrix-valued function defined by the formula

$$
L_{Q}(\boldsymbol{\theta}):=|\Omega|^{-1} \int_{\Omega}\left(\Lambda_{Q}(\mathbf{x})^{*} b(\boldsymbol{\theta})^{*} \widetilde{g}(\mathbf{x})+\widetilde{g}(\mathbf{x})^{*} b(\boldsymbol{\theta}) \Lambda_{Q}(\mathbf{x})\right) d \mathbf{x} .
$$

Note that $L_{Q}(\mathbf{k})=t L_{Q}(\boldsymbol{\theta})$ is the symbol of a selfadjoint homogeneous first-order DO $L_{Q}(\mathbf{D})$. We put

$$
\widehat{N}_{Q}(\mathbf{k}):=t^{3} \widehat{N}_{Q}(\boldsymbol{\theta})=b(\mathbf{k})^{*} L_{Q}(\mathbf{k}) b(\mathbf{k}) \widehat{P}, \quad \mathbf{k}=t \boldsymbol{\theta} .
$$

The matrix-valued function $b(\mathbf{k})^{*} L_{Q}(\mathbf{k}) b(\mathbf{k})$ is the symbol of the homogeneous selfadjoint third-order DO $b(\mathbf{D})^{*} L_{Q}(\mathbf{D}) b(\mathbf{D})$. The operators $\widehat{Z}_{Q}(\boldsymbol{\theta})$ and $\widehat{N}_{Q}(\boldsymbol{\theta})$ admit the following estimates uniform in $\boldsymbol{\theta}$ (see [6, (5.9), (5.15)]):

$$
\begin{aligned}
\left\|\hat{Z}_{Q}(\boldsymbol{\theta})\right\| & \leq 2^{-1 / 2} \alpha_{0}^{-1 / 2} \alpha_{1}^{1 / 2} r_{0}^{-1}\|g\|_{L_{\infty}}^{1 / 2}\left\|g^{-1}\right\|_{L_{\infty}}^{-1 / 2}\|f\|_{L_{\infty}}^{2}\left\|f^{-1}\right\|_{L_{\infty}}^{2}, \\
\left\|\hat{N}_{Q}(\boldsymbol{\theta})\right\| & \leq 2^{1 / 2} \alpha_{0}^{-1 / 2} \alpha_{1}^{3 / 2} r_{0}^{-1}\|g\|_{L_{\infty}}^{3 / 2}\left\|g^{-1}\right\|_{L_{\infty}}^{1 / 2}\|f\|_{L_{\infty}}^{2}\left\|f^{-1}\right\|_{L_{\infty}}^{2} .
\end{aligned}
$$

$\S 6$. Approximation of the "Bordered" operator exponential $f e^{-\mathcal{A}(\mathbf{k})} f^{*}$

6.1. Approximation of the operator $f e^{-\mathcal{A}(\mathbf{k}) \tau} f^{*}$ for $|\mathbf{k}| \leq t^{0}$. By (4.20), (4.21) the kernel $\hat{\mathfrak{N}}$ and the orthogonal projection $\widehat{P}$ do not depend on $\boldsymbol{\theta}$. Moreover, the constants $D_{0}(\boldsymbol{\theta})$ and $D_{1}(\boldsymbol{\theta})$ defined in (2.21), (2.24) can be majorized uniformly in $\boldsymbol{\theta}$. For that, we substitute in (2.21) the values of $c_{*}$ and $\delta$ from (4.13), (4.14) and use (4.15). Recalling 
E. S. VASILEVSKAYA

the definitions of the constants $C_{1}, C_{2}, C_{5}, C_{6}$ and denoting $\alpha_{1}\|g\|_{L_{\infty}}\|f\|_{L_{\infty}}^{2} \alpha=: x$, we obtain

$$
\begin{aligned}
\left|D_{0}(\boldsymbol{\theta})\right| \leq & \mathcal{D}_{0}, \\
\mathcal{D}_{0}:= & 4 \alpha_{0}^{-1}\left\|g^{-1}\right\|_{L_{\infty}}\left\|f^{-1}\right\|_{L_{\infty}}^{2} r_{0}^{-2} \\
& \times \max _{x \geq 0}\left\{1+\varphi(x) \exp \left\{-c_{0} c_{1}^{-1}\|f\|_{L_{\infty}}^{-2}\left\|f^{-1}\right\|_{L_{\infty}}^{-2} x\right\}\right\}, \\
\varphi(x):= & \sqrt{2}\left(\beta_{1}+\sqrt{2} \beta^{(2)}\right) x+\left(\beta+2 \sqrt{2} \beta_{1}+2^{-1 / 2} \beta_{2}\right) x^{2}+2^{-1 / 2} \beta_{2} x^{3},
\end{aligned}
$$

where $c_{0}, c_{1}$ are the constants defined in (4.5). The constants $D_{1}(\boldsymbol{\theta})$ (see (2.24)) are bounded uniformly in $\boldsymbol{\theta}$ :

$$
\left|D_{1}(\boldsymbol{\theta})\right| \leq \mathcal{D}_{1}, \quad \mathcal{D}_{1}:=\max \left\{2 \mathcal{D}_{0}, 4+\sqrt{2}+8^{-1 / 2} \alpha_{0}\left\|g^{-1}\right\|_{L_{\infty}}^{-1}\left\|f^{-1}\right\|_{L_{\infty}}^{-2} r_{0}^{2}\right\} .
$$

We apply Theorem 3.1 to the operator $\mathcal{A}(\mathbf{k})$ with $|\mathbf{k}| \leq t^{0}$, obtaining

$$
\begin{aligned}
f e^{-\mathcal{A}(\mathbf{k}) \tau} f^{*}= & f_{0} e^{-t^{2} \tau f_{0} \widehat{S}(\boldsymbol{\theta}) f_{0}} f_{0} \widehat{P} \\
& +t\left(\widehat{Z}_{Q}(\boldsymbol{\theta}) f_{0} e^{-t^{2} \tau f_{0} \widehat{S}(\boldsymbol{\theta}) f_{0}} f_{0} \widehat{P}+f_{0} e^{-t^{2} \tau f_{0} \widehat{S}(\boldsymbol{\theta}) f_{0}} f_{0} \widehat{P} \widehat{Z}_{Q}(\boldsymbol{\theta})^{*}\right) \\
& -t^{3} \int_{0}^{\tau} f_{0} e^{-t^{2}(\tau-\widetilde{\tau}) f_{0} \widehat{S}(\boldsymbol{\theta}) f_{0}} f_{0} \widehat{N}_{Q}(\boldsymbol{\theta}) f_{0} e^{-t^{2} \widetilde{\tau} f_{0} \widehat{S}(\boldsymbol{\theta}) f_{0}} f_{0} \widehat{P} d \widetilde{\tau} \\
& +\mathcal{Y}(t, \boldsymbol{\theta}, \tau), \quad|\mathbf{k}| \leq t^{0},
\end{aligned}
$$

where $f_{0}$ is the matrix defined in (4.24), and the operators $\mathcal{Y}(t, \boldsymbol{\theta}, \tau)$ admit the estimates

$$
\begin{array}{lll}
\|\mathcal{Y}(t, \boldsymbol{\theta}, \tau)\| \leq\|f\|_{L_{\infty}}^{2} \mathcal{D}_{0} \tau^{-1}, & & \tau>0 \\
\|\mathcal{Y}(t, \boldsymbol{\theta}, \tau)\| \leq\|f\|_{L_{\infty}}^{2} \mathcal{D}_{1}(1+\tau)^{-1}, & & \tau \geq 0,
\end{array}
$$

with the same constants $\mathcal{D}_{0}, \mathcal{D}_{1}$ as in (6.1), (6.2); these constants do not depend on $\boldsymbol{\theta}$.

6.2. Approximation of the operator $f e^{-\mathcal{A}(\mathbf{k}) \tau} f^{*}$ for $\mathbf{k} \in \widetilde{\Omega} \backslash \mathcal{B}\left(t^{0}\right)$. The estimates for $|\mathbf{k}|>t^{0}$ are rough: each term in (6.3) is estimated individually and all of them can be neglected. We explain this. Put

$$
2 r_{1}:=\operatorname{diam} \widetilde{\Omega} ; \quad \mathcal{B}\left(t^{0}\right):=\left\{\mathbf{k} \in \mathbb{R}^{d}:|\mathbf{k}| \leq t^{0}\right\} .
$$

For $\mathbf{k} \in \widetilde{\Omega} \backslash \mathcal{B}\left(t^{0}\right)$, the inequality $e^{-\alpha} \leq(1+\alpha)^{-1}$ implies the required estimates for $\tau \geq 0$. By (4.17), we conclude that

$$
\left\|f e^{-\mathcal{A}(\mathbf{k}) \tau} f^{*}\right\| \leq\|f\|_{L_{\infty}}^{2}\left(1+c_{*}\left(t^{0}\right)^{2} \tau\right)^{-1}, \quad \tau \geq 0
$$

By (3.3), we have

$$
f_{0} e^{-t^{2} \tau f_{0} \hat{S}(\boldsymbol{\theta}) f_{0}} f_{0} \widehat{P}=f e^{-t^{2} \tau S(\boldsymbol{\theta})} P f^{*},
$$

and (4.18) implies that

$$
\left\|f_{0} e^{-t^{2} \tau f_{0} \hat{S}(\boldsymbol{\theta}) f_{0}} f_{0} \hat{P}\right\| \leq\|f\|_{L_{\infty}}^{2}\left(1+c_{*}\left(t^{0}\right)^{2} \tau\right)^{-1}, \quad \tau \geq 0 .
$$

Further, using the inequality $e^{-\alpha} \alpha \leq 2(1+\alpha)^{-1}$, for $\tau \geq 0$ we obtain

$$
\begin{aligned}
& \left\|\int_{0}^{\tau} f_{0} e^{-t^{2}(\tau-\widetilde{\tau}) f_{0} \hat{S}(\boldsymbol{\theta}) f_{0}} f_{0} \hat{N}_{Q}(\boldsymbol{\theta}) f_{0} e^{-t^{2} \widetilde{\tau} f_{0} \widehat{S}(\boldsymbol{\theta}) f_{0}} f_{0} \hat{P} d \widetilde{\tau}\right\| \\
& \quad \leq\|f\|_{L_{\infty}}^{4}\left\|\hat{N}_{Q}(\boldsymbol{\theta})\right\| \int_{0}^{\tau} e^{-c_{*} t^{2}(\tau-\widetilde{\tau})} e^{-c_{*} t^{2} \widetilde{\tau}} d \widetilde{\tau} \\
& \quad \leq 2\|f\|_{L_{\infty}}^{4}\left\|\hat{N}_{Q}(\boldsymbol{\theta})\right\|\left(c_{*}\left(t^{0}\right)^{2}\right)^{-1}\left(1+c_{*}\left(t^{0}\right)^{2} \tau\right)^{-1} .
\end{aligned}
$$


The inequalities $e^{-\alpha} \leq \alpha^{-1}$ and $e^{-\alpha} \leq 2 \alpha^{-2}$ imply similar estimates for $\tau>0$ :

$$
\begin{aligned}
& \left\|f e^{-\mathcal{A}(\mathbf{k}) \tau} f^{*}\right\| \leq\|f\|_{L_{\infty}}^{2} c_{*}^{-1}\left(t^{0}\right)^{-2} \tau^{-1}, \\
& \left\|f_{0} e^{-t^{2} \tau f_{0} \widehat{S}(\boldsymbol{\theta}) f_{0}} f_{0} \widehat{P}\right\| \leq\|f\|_{L_{\infty}}^{2} c_{*}^{-1}\left(t^{0}\right)^{-2} \tau^{-1}, \\
& \left\|\int_{0}^{\tau} f_{0} e^{-t^{2}(\tau-\widetilde{\tau}) f_{0} \hat{S}(\boldsymbol{\theta}) f_{0}} f_{0} \hat{N}_{Q}(\boldsymbol{\theta}) f_{0} e^{-t^{2} \widetilde{\tau} f_{0} \hat{S}(\boldsymbol{\theta}) f_{0}} f_{0} \widehat{P} d \widetilde{\tau}\right\| \\
& \leq 2\|f\|_{L_{\infty}}^{4}\left\|\hat{N}_{Q}(\boldsymbol{\theta})\right\|\left(c_{*}\left(t^{0}\right)^{2}\right)^{-2} \tau^{-1} .
\end{aligned}
$$

Relations (5.10), (5.11), (4.13), (4.14), (4.16), and (4.5) show that, for $\mathbf{k} \in \widetilde{\Omega} \backslash \mathcal{B}\left(t^{0}\right)$, formula (6.3) is valid with the estimates

$$
\begin{aligned}
& \|\mathcal{Y}(t, \boldsymbol{\theta}, \tau)\| \leq \mathcal{D}_{2} \tau^{-1}, \quad \tau>0, \\
& \mathcal{D}_{2}:=8 c_{0}^{-2} c_{1}\|f\|_{L_{\infty}}^{4}\left\|f^{-1}\right\|_{L_{\infty}}^{4} r_{0}^{-2} \\
& \times\left(1+\left(2^{-1} c_{0}^{-1} c_{1}\right)^{1 / 2} r_{0}^{-1} r_{1}\|f\|_{L_{\infty}}^{2}\left\|f^{-1}\right\|_{L_{\infty}}^{2}\right. \\
& \left.+\left(2 c_{0}^{-1} c_{1}\right)^{5 / 2}\left(r_{0}^{-1} r_{1}\|f\|_{L_{\infty}}^{2}\left\|f^{-1}\right\|_{L_{\infty}}^{2}\right)^{3}\right), \\
& \|\mathcal{Y}(t, \boldsymbol{\theta}, \tau)\| \leq \mathcal{D}_{3}(1+\tau)^{-1}, \quad \tau \geq 0, \\
& \mathcal{D}_{3}:=\mathcal{D}_{2} \max \left\{1,4^{-1} c_{0}^{2} c_{1}^{-1}\|f\|_{L_{\infty}}^{-2}\left\|f^{-1}\right\|_{L_{\infty}}^{-4} r_{0}^{2}\right\} .
\end{aligned}
$$

Thus, we arrive at the following result.

Theorem 6.1. For $\mathbf{k}=t \boldsymbol{\theta} \in \widetilde{\Omega}$ and $\tau \geq 0$, we have

$$
\begin{aligned}
f e^{-\mathcal{A}(\mathbf{k}) \tau} f^{*}= & f_{0} e^{-\tau f_{0} \hat{S}(\mathbf{k}) f_{0}} f_{0} \widehat{P} \\
& +\widehat{Z}_{Q}(\mathbf{k}) f_{0} e^{-\tau f_{0} \widehat{S}(\mathbf{k}) f_{0}} f_{0} \widehat{P}+f_{0} e^{-\tau f_{0} \widehat{S}(\mathbf{k}) f_{0}} f_{0} \widehat{P} \hat{Z}_{Q}(\mathbf{k})^{*} \\
& -\int_{0}^{\tau} f_{0} e^{-(\tau-\widetilde{\tau}) f_{0} \hat{S}(\mathbf{k}) f_{0}} f_{0} \hat{N}_{Q}(\mathbf{k}) f_{0} e^{-\widetilde{\tau} f_{0} \widehat{S}(\mathbf{k}) f_{0}} f_{0} \widehat{P} d \widetilde{\tau}+\mathcal{Y}(t, \boldsymbol{\theta}, \tau),
\end{aligned}
$$

where

$$
\begin{array}{lll}
\|\mathcal{Y}(t, \boldsymbol{\theta}, \tau)\|_{L_{2}(\Omega) \rightarrow L_{2}(\Omega)} \leq \mathcal{D}_{4} \tau^{-1}, & \tau>0, & \mathcal{D}_{4}:=\max \left\{\|f\|_{L_{\infty}}^{2} \mathcal{D}_{0}, \mathcal{D}_{2}\right\}, \\
\|\mathcal{Y}(t, \boldsymbol{\theta}, \tau)\|_{L_{2}(\Omega) \rightarrow L_{2}(\Omega)} \leq \mathcal{D}_{5}(1+\tau)^{-1}, & \tau \geq 0, & \mathcal{D}_{5}:=\max \left\{\|f\|_{L_{\infty}}^{2} \mathcal{D}_{1}, \mathcal{D}_{3}\right\} .
\end{array}
$$

Here $\mathcal{D}_{0}, \mathcal{D}_{1}, \mathcal{D}_{2}, \mathcal{D}_{3}$ are the constants occurring in (6.1), (6.2), (6.4), and (6.5).

§7. Approximation of the "Bordered" operator exponential $f e^{-\mathcal{A} \tau} f^{*}$

7.1. Approximation of the operator $f e^{-\mathcal{A} \tau} f^{*}$. Let $\mathcal{A}(\mathbf{k}), \widehat{\mathcal{A}}^{0}(\mathbf{k})$ be the operators that arise in the expansion of the operators $(4.4),(5.6)$ in a direct integral. Then relation (5.5) and the fact that $\widehat{P}$ is the orthogonal projection onto the kernel $\hat{\mathfrak{N}}=\left\{\mathbf{u}=\mathbf{c} \in \mathbb{C}^{n}\right\}$ show that $e^{-f_{0} \hat{S}(\mathbf{k}) f_{0} \tau} \widehat{P}=e^{-f_{0} \hat{\mathcal{A}}^{0}(\mathbf{k}) f_{0} \tau} \hat{P}$.

We introduce the notation

$$
\mathcal{A}^{0}:=f_{0} \widehat{\mathcal{A}}^{0} f_{0}, \quad \mathcal{A}^{0}(\mathbf{k}):=f_{0} \widehat{\mathcal{A}}^{0}(\mathbf{k}) f_{0}
$$

and rewrite (6.6) as follows:

$$
\begin{aligned}
f e^{-\mathcal{A}(\mathbf{k}) \tau} f^{*}= & f_{0} e^{-\mathcal{A}^{0}(\mathbf{k}) \tau} f_{0} \widehat{P}+\widehat{Z}_{Q}(\mathbf{k}) f_{0} e^{-\mathcal{A}^{0}(\mathbf{k}) \tau} f_{0} \widehat{P}+f_{0} e^{-\mathcal{A}^{0}(\mathbf{k}) \tau} f_{0} \widehat{P} \widehat{Z}_{Q}(\mathbf{k})^{*} \\
& -\int_{0}^{\tau} f_{0} e^{-\mathcal{A}^{0}(\mathbf{k})(\tau-\widetilde{\tau})} f_{0} \widehat{N}_{Q}(\mathbf{k}) f_{0} e^{-\mathcal{A}^{0}(\mathbf{k}) \widetilde{\tau}} f_{0} \widehat{P} d \widetilde{\tau}+\mathcal{Y}(\mathbf{k}, \tau), \quad \mathbf{k} \in \widetilde{\Omega} .
\end{aligned}
$$


E. S. VASILEVSKAYA

Using the presence of the projection $\widehat{P}$ once again, we replace the operator $\hat{Z}_{Q}(\mathbf{k})=$ $\Lambda_{Q} b(\mathbf{k}) \hat{P}$ by $\Lambda_{Q} b(\mathbf{D}+\mathbf{k}) \hat{P}$ and the operator $\hat{N}_{Q}(\mathbf{k}) \hat{P}=b(\mathbf{k})^{*} L_{Q}(\mathbf{k}) b(\mathbf{k}) \hat{P}$ by $b(\mathbf{D}+\mathbf{k})^{*} L_{Q}(\mathbf{D}+\mathbf{k}) b(\mathbf{D}+\mathbf{k}) \hat{P}$. Then for $\mathbf{k} \in \widetilde{\Omega}$ we have

$$
\begin{aligned}
f e^{-\mathcal{A}(\mathbf{k}) \tau} f^{*}= & f_{0} e^{-\mathcal{A}^{0}(\mathbf{k}) \tau} f_{0} \widehat{P}+\Lambda_{Q} b(\mathbf{D}+\mathbf{k}) f_{0} e^{-\mathcal{A}^{0}(\mathbf{k}) \tau} f_{0} \widehat{P} \\
& +f_{0} e^{-\mathcal{A}^{0}(\mathbf{k}) \tau} f_{0} \widehat{P}\left(\Lambda_{Q} b(\mathbf{D}+\mathbf{k}) \widehat{P}\right)^{*} \\
& -\int_{0}^{\tau} f_{0} e^{-\mathcal{A}^{0}(\mathbf{k})(\tau-\widetilde{\tau})} f_{0} \widehat{P} b(\mathbf{D}+\mathbf{k})^{*} L_{Q}(\mathbf{D}+\mathbf{k}) b(\mathbf{D}+\mathbf{k}) \widehat{P} f_{0} e^{-\mathcal{A}^{0}(\mathbf{k}) \tilde{\tau}} f_{0} d \widetilde{\tau} \\
& +\mathcal{Y}(\mathbf{k}, \tau) .
\end{aligned}
$$

Here

$$
\begin{aligned}
\|\mathcal{Y}(\mathbf{k}, \tau)\| \leq \mathcal{D}_{4} \tau^{-1}, & & \tau>0, & \mathbf{k} \in \widetilde{\Omega}, \\
\|\mathcal{Y}(\mathbf{k}, \tau)\| \leq \mathcal{D}_{5}(1+\tau)^{-1}, & & \tau \geq 0, & \mathbf{k} \in \widetilde{\Omega} .
\end{aligned}
$$

The operator $e^{-\mathcal{A} \tau}$ is approximated with the help of the formula

$$
e^{-\mathcal{A} \tau}=\mathcal{U}^{-1}\left(\int_{\tilde{\Omega}} \oplus e^{-\mathcal{A}(\mathbf{k}) \tau} d \mathbf{k}\right) \mathcal{U}
$$

which is a consequence of (4.9). We denote (see [6, (6.7)])

$$
\Pi:=\mathcal{U}^{-1}[\hat{P}] \mathcal{U},
$$

where $[\widehat{P}]$ is the orthogonal projection in $\mathcal{H}$ (see (4.6)) that acts in the layers as the operator $\widehat{P}$. Then (7.2) turns into the relation

$$
\begin{aligned}
f e^{-\mathcal{A} \tau} f^{*}= & \mathcal{E}^{0}(\tau) \Pi+\Lambda_{Q} b(\mathbf{D}) \Pi \mathcal{E}^{0}(\tau)+\mathcal{E}^{0}(\tau)\left(\Lambda_{Q} b(\mathbf{D}) \Pi\right)^{*} \\
& -\int_{0}^{\tau} \mathcal{E}^{0}(\tau-\widetilde{\tau}) b(\mathbf{D})^{*} L_{Q}(\mathbf{D}) b(\mathbf{D}) \Pi \mathcal{E}^{0}(\widetilde{\tau}) d \widetilde{\tau}+\mathcal{J}(\tau),
\end{aligned}
$$

where

$$
\mathcal{E}^{0}(\tau):=f_{0} e^{-\mathcal{A}^{0} \tau} f_{0}
$$

Since $\mathcal{U}$ is unitary, for the operator $\mathcal{J}(\tau)$ we have

$$
\begin{aligned}
\|\mathcal{J}(\tau)\| & \leq \mathcal{D}_{4} \tau^{-1}, & & \tau>0, \\
\|\mathcal{J}(\tau)\| & \leq \mathcal{D}_{5}(1+\tau)^{-1}, & & \tau \geq 0,
\end{aligned}
$$

with constants $\mathcal{D}_{4}$ and $\mathcal{D}_{5}$ as defined in (6.7).

7.2. "Elimination" of the operator $\Pi$. Now we study the possibility of replacing $\Pi$ by the identity operator $I$ in (7.3). We start with the first term and estimate the norm of the operator $\mathcal{E}^{0}(\tau)(I-\Pi)$. For that, we consider its symbol $\sigma^{(1)}(\boldsymbol{\xi} ; \tau)$. As was shown in [6. (6.8)],

$$
\Pi=\mathcal{F}^{*}\left[\chi_{\Omega}\right] \mathcal{F},
$$

where $\left[\chi_{\widetilde{\Omega}}\right]$ is the operator of multiplication by the characteristic function of the set $\widetilde{\Omega}$ and $\mathcal{F}$ is the Fourier operator. Then

$$
\sigma^{(1)}(\boldsymbol{\xi} ; \tau)=f_{0} e^{-\tau f_{0} b(\boldsymbol{\xi})^{*} g^{0} b(\boldsymbol{\xi}) f_{0}} f_{0}\left(1-\chi_{\widetilde{\Omega}}(\boldsymbol{\xi})\right) .
$$

From (4.24) it follows that

$$
\left\|f_{0}\right\|_{L_{\infty}} \leq\|f\|_{L_{\infty}}, \quad\left\|f_{0}^{-1}\right\|_{L_{\infty}} \leq\left\|f^{-1}\right\|_{L_{\infty}} .
$$


Using (4.2) and the inequality

$$
\left\|\left(g^{0}\right)^{-1}\right\|_{L_{\infty}} \leq\left\|g^{-1}\right\|_{L_{\infty}}
$$

(see [6, (4.18)]), we obtain

$$
f_{0} b(\boldsymbol{\xi})^{*} g^{0} b(\boldsymbol{\xi}) f_{0} \geq \alpha_{0}|\boldsymbol{\xi}|^{2}\left\|g^{-1}\right\|_{L_{\infty}}^{-1}\left\|f^{-1}\right\|_{L_{\infty}}^{-2} \mathbf{1}_{n}=c_{*}|\boldsymbol{\xi}|^{2} \mathbf{1}_{n}, \quad \boldsymbol{\xi} \in \mathbb{R}^{d} .
$$

Therefore, the inequality $e^{-\alpha} \leq(1+\alpha)^{-1}$ yields the estimate

$$
\left|\sigma^{(1)}(\boldsymbol{\xi} ; \tau)\right| \leq\|f\|_{L_{\infty}}^{2}\left(1+c_{*}|\boldsymbol{\xi}|^{2} \tau\right)^{-1}\left(1-\chi_{\widetilde{\Omega}}(\boldsymbol{\xi})\right) .
$$

Since for $\boldsymbol{\xi} \in \mathbb{R}^{d} \backslash \widetilde{\Omega}$ we have $|\boldsymbol{\xi}| \geq r_{0}$, it follows that

$$
\begin{array}{ll}
\left\|\mathcal{E}^{0}(\tau)(I-\Pi)\right\|=\sup _{\boldsymbol{\xi} \in \mathbb{R}^{d}}\left|\sigma^{(1)}(\boldsymbol{\xi} ; \tau)\right| \leq \eta^{(1)} \tau^{-1}, & \tau>0, \\
\left\|\mathcal{E}^{0}(\tau)(I-\Pi)\right\| \leq \eta^{(2)}(1+\tau)^{-1}, & \tau \geq 0
\end{array}
$$

where

$$
\eta^{(1)}:=c_{0}^{-1} r_{0}^{-2}\|f\|_{L_{\infty}}^{2}\left\|f^{-1}\right\|_{L_{\infty}}^{2}, \quad \eta^{(2)}:=\max \left\{\|f\|_{L_{\infty}}^{2}, \eta^{(1)}\right\} .
$$

Consider the fourth term in (7.3). We need to estimate the norm of the operator

$$
\int_{0}^{\tau} \mathcal{E}^{0}(\tau-\tilde{\tau}) b(\mathbf{D})^{*} L_{Q}(\mathbf{D}) b(\mathbf{D})(I-\Pi) \mathcal{E}^{0}(\widetilde{\tau}) d \tilde{\tau} .
$$

Let $\sigma^{(2)}(\boldsymbol{\xi} ; \tau)$ denote its symbol:

$\sigma^{(2)}(\boldsymbol{\xi} ; \tau)$

$$
=\int_{0}^{\tau} f_{0} e^{-(\tau-\widetilde{\tau}) f_{0} b(\boldsymbol{\xi})^{*} g^{0} b(\boldsymbol{\xi}) f_{0}} f_{0} b(\boldsymbol{\xi})^{*} L_{Q}(\boldsymbol{\xi}) b(\boldsymbol{\xi}) f_{0} e^{-\widetilde{\tau} f_{0} b(\boldsymbol{\xi})^{*} g^{0} b(\boldsymbol{\xi}) f_{0}} f_{0}\left(1-\chi_{\widetilde{\Omega}}(\boldsymbol{\xi})\right) d \tilde{\tau} .
$$

Using (5.9), (5.11), (7.7), (7.8), (4.5), (4.13) and the inequality $x^{2} e^{-x} \leq 1$ for $\tau>0$, $\boldsymbol{\xi} \in \mathbb{R}^{d}$, we obtain

$$
\left|\sigma^{(2)}(\boldsymbol{\xi} ; \tau)\right| \leq \sqrt{2} c_{0}^{-1 / 2} c_{1}^{3 / 2} r_{0}^{-1}\|f\|_{L_{\infty}}^{6}\left\|f^{-1}\right\|_{L_{\infty}}^{2}|\boldsymbol{\xi}|^{3} \tau e^{-c_{*}|\boldsymbol{\xi}|^{2} \tau}\left(1-\chi_{\widetilde{\Omega}}(\boldsymbol{\xi})\right) \leq \eta^{(3)} \tau^{-1},
$$

where

$$
\eta^{(3)}:=\sqrt{2} c_{0}^{-5 / 2} c_{1}^{3 / 2} r_{0}^{-2}\|f\|_{L_{\infty}}^{6}\left\|f^{-1}\right\|_{L_{\infty}}^{6}
$$

Hence,

$$
\left\|\int_{0}^{\tau} \mathcal{E}^{0}(\tau-\tilde{\tau}) b(\mathbf{D})^{*} L(\mathbf{D}) b(\mathbf{D})(I-\Pi) \mathcal{E}^{0}(\tilde{\tau}) d \widetilde{\tau}\right\| \leq \eta^{(3)} \tau^{-1}, \quad \tau>0 .
$$

Consider the second term in (7.3). (The third term is adjoint to the second and does not require any special consideration.) We estimate the norm of the operator $\Lambda_{Q} b(\mathbf{D})(I-\Pi) \mathcal{E}^{0}(\tau)$. Using $(5.7)$, we write

$$
\Lambda_{Q} b(\mathbf{D})(I-\Pi) \mathcal{E}^{0}(\tau)=\Lambda b(\mathbf{D})(I-\Pi) \mathcal{E}^{0}(\tau)+\Lambda_{Q}^{0} b(\mathbf{D})(I-\Pi) \mathcal{E}^{0}(\tau) .
$$

The second term in (7.14) is estimated by using [6, (7.14)]:

$$
\left\|\Lambda_{Q}^{0}\right\| \leq m^{1 / 2}\left(2 r_{0}\right)^{-1} \alpha_{0}^{-1 / 2}\|g\|_{L_{\infty}}^{1 / 2}\left\|g^{-1}\right\|_{L_{\infty}}^{1 / 2}\|Q\|_{L_{\infty}}\left\|Q^{-1}\right\|_{L_{\infty}} .
$$

Moreover,

$$
\left\|b(\mathbf{D})(I-\Pi) \mathcal{E}^{0}(\tau)\right\|=\sup _{\boldsymbol{\xi} \in \mathbb{R}^{d}}\left|b(\boldsymbol{\xi}) \sigma^{(1)}(\boldsymbol{\xi})\right| \leq \alpha_{1}^{1 / 2} c_{*}^{-1} r_{0}^{-1}\|f\|_{L_{\infty}}^{2} \tau^{-1} .
$$


Then

$$
\begin{aligned}
& \left\|\Lambda_{Q}^{0} b(\mathbf{D})(I-\Pi) \mathcal{E}^{0}(\tau)\right\| \leq \lambda_{0} \tau^{-1}, \quad \tau>0, \\
& \lambda_{0}:=2^{-1} m^{1 / 2} c_{0}^{-3 / 2} c_{1}^{1 / 2} r_{0}^{-2}\|f\|_{L_{\infty}}^{4}\left\|f^{-1}\right\|_{L_{\infty}}^{4} .
\end{aligned}
$$

To estimate the norm of the operator $\Lambda b(\mathbf{D})(I-\Pi) \mathcal{E}^{0}(\tau)$, first we show that the operator $b(\mathbf{D})(I-\Pi) \mathcal{E}^{0}(\tau)$ is a continuous operator from $L_{2}\left(\mathbb{R}^{d} ; \mathbb{C}^{n}\right)$ to $H^{\ell}\left(\mathbb{R}^{d} ; \mathbb{C}^{m}\right)$ for any $\ell \geq 1$. Indeed, using (4.2), (7.7), (7.8), and the inequality $e^{-x} \leq \mathcal{C}(\ell) x^{-(\ell+1) / 2}$, we see that

$$
\begin{aligned}
& \left\|b(\mathbf{D})(I-\Pi) \mathcal{E}^{0}(\tau)\right\|_{L_{2}\left(\mathbb{R}^{d} ; \mathbb{C}^{n}\right) \rightarrow H^{\ell}\left(\mathbb{R}^{d} ; \mathbb{C}^{m}\right)} \\
& \quad=\sup _{\boldsymbol{\xi} \in \mathbb{R}^{d}}\left(1+|\boldsymbol{\xi}|^{2}\right)^{\ell / 2}\left|b(\boldsymbol{\xi}) \sigma^{(1)}(\boldsymbol{\xi} ; \tau)\right|\left(1-\chi_{\widetilde{\Omega}}(\boldsymbol{\xi})\right) \\
& \quad \leq \alpha_{1}^{1 / 2} \sup _{\boldsymbol{\xi} \in \mathbb{R}^{d}}|\boldsymbol{\xi}|\left(1+|\boldsymbol{\xi}|^{2}\right)^{\ell / 2}\|f\|_{L_{\infty}}^{2} e^{-c_{*}|\boldsymbol{\xi}|^{2} \tau}\left(1-\chi_{\widetilde{\Omega}}(\boldsymbol{\xi})\right) \\
& \quad \leq \alpha_{1}^{1 / 2} c_{*}^{-(\ell+1) / 2}\|f\|_{L_{\infty}}^{2} \mathcal{C}(\ell)\left(1+r_{0}^{-2}\right)^{\ell / 2} \tau^{-(\ell+1) / 2}, \quad \tau>0 .
\end{aligned}
$$

Since in what follows we are interested in the case where $\tau \rightarrow \infty$, it suffices to assume that $\tau \geq 1$. Then

$$
\begin{aligned}
& \left\|b(\mathbf{D})(I-\Pi) \mathcal{E}^{0}(\tau)\right\|_{L_{2}\left(\mathbb{R}^{d}\right) \rightarrow H^{\ell}\left(\mathbb{R}^{d}\right)} \\
& \quad \leq \alpha_{1}^{1 / 2} c_{*}^{-(\ell+1) / 2}\|f\|_{L_{\infty}}^{2} \mathcal{C}(\ell)\left(1+r_{0}^{-2}\right)^{\ell / 2} \tau^{-1}, \quad \ell \geq 1, \quad \tau \geq 1 .
\end{aligned}
$$

It remains to show that $\Lambda$ acts continuously from $H^{\ell}\left(\mathbb{R}^{d} ; \mathbb{C}^{m}\right)$ to $L_{2}\left(\mathbb{R}^{d} ; \mathbb{C}^{n}\right)$ for some $\ell \geq 1$. For the dimensions $d \leq 4$, this fact was shown in 6, Subsection 6.3] for $\ell=$ 1. In Proposition 6.8 of [6] it was shown, in particular, that in this case the norm $\|\Lambda\|_{H^{1}\left(\mathbb{R}^{d} ; \mathbb{C}^{m}\right) \rightarrow L_{2}\left(\mathbb{R}^{d} ; \mathbb{C}^{n}\right)}$ is dominated by a quantity depending only on $\|g\|_{L_{\infty}},\left\|g^{-1}\right\|_{L_{\infty}}$, $\alpha_{0}, m, n, d$, and on the lattice $\Gamma$.

We show that a similar assertion is valid in the case of an arbitrary dimension $(d>4)$ for $\ell=d / 2-1$. Suppose $d>4$. Since the columns $\mathbf{v}_{j}(\mathbf{x})$ of the matrix $\Lambda(\mathbf{x})$ belong to $\widetilde{H}^{1}\left(\Omega ; \mathbb{C}^{n}\right)$ (see $(5.1)$ ) and since $H^{1}(\Omega) \subset L_{2 d /(d-2)}(\Omega)$, it follows that $\Lambda \in L_{2 d /(d-2)}(\Omega)$. Suppose $\mathbf{a} \in \Gamma, \mathbf{v} \in H^{\ell}\left(\mathbb{R}^{d} ; \mathbb{C}^{m}\right)$, and $\ell=d / 2-1$. Then, since the matrix $\Lambda(\mathbf{x})$ is periodic with respect to the lattice $\Gamma$, we obtain

$$
\int_{\Omega+\mathbf{a}}|\Lambda(\mathbf{x}) \mathbf{v}(\mathbf{x})|^{2} d \mathbf{x} \leq\|\Lambda\|_{L_{q}(\Omega)}^{2}\|\mathbf{v}\|_{L_{d}(\Omega+\mathbf{a})}^{2}
$$

where $q:=2 d /(d-2)$. The inclusion $H^{\ell}(\Omega) \subset L_{d}(\Omega)$ for $\ell=d / 2-1$ implies that

$$
\|\mathbf{v}\|_{L_{d}\left(\Omega+\mathbf{a} ; \mathbb{C}^{m}\right)} \leq c(m, d, \Omega)\|\mathbf{v}\|_{H^{\ell}\left(\Omega+\mathbf{a} ; \mathbb{C}^{m}\right)} .
$$

Substituting (7.18) in (7.17) and summing over all $\mathbf{a} \in \Gamma$, we arrive at the inequality

$$
\int_{\mathbb{R}^{d}}|\Lambda(\mathbf{x}) \mathbf{v}(\mathbf{x})|^{2} d \mathbf{x} \leq c^{2}(m, d, \Omega)\|\Lambda\|_{L_{q}(\Omega)}^{2}\|\mathbf{v}\|_{H^{\ell}\left(\mathbb{R}^{d} ; \mathbb{C}^{m}\right)}^{2}
$$

which shows that

$$
\|\Lambda\|_{H^{\ell}\left(\mathbb{R}^{d} ; \mathbb{C}^{m}\right) \rightarrow L_{2}\left(\mathbb{R}^{d} ; \mathbb{C}^{n}\right)} \leq c(m, d, \Omega)\|\Lambda\|_{L_{q}(\Omega)} .
$$

The inclusion $H^{1}(\Omega) \subset L_{q}(\Omega)$ yields

$$
\|\Lambda\|_{L_{q}(\Omega)} \leq \widetilde{c}(d, m, n, \Omega)\|\Lambda\|_{H^{1}(\Omega)},
$$

and therefore,

$$
\begin{aligned}
\|\Lambda\|_{H^{\ell}\left(\mathbb{R}^{d} ; \mathbb{C}^{m}\right) \rightarrow L_{2}\left(\mathbb{R}^{d} ; \mathbb{C}^{n}\right)} & \leq \widehat{c}(d, m, n, \Omega)\|\Lambda\|_{H^{1}(\Omega)}, \quad \ell=d / 2-1, \\
\widehat{c}(d, m, n, \Omega) & :=c(d, m, \Omega) \widetilde{c}(d, m, n, \Omega) .
\end{aligned}
$$


The estimate

$$
\|\Lambda\|_{H^{1}(\Omega)} \leq m^{1 / 2}\left(1+1 / 4 r_{0}^{2}\right)^{1 / 2} \alpha_{0}^{-1 / 2}|\Omega|^{1 / 2}\|g\|_{L_{\infty}}^{1 / 2}\left\|g^{-1}\right\|_{L_{\infty}}^{1 / 2}
$$

was obtained in [6, (6.30)]; this implies, together with (7.19), that the norm

$$
\|\Lambda\|_{H^{\ell}\left(\mathbb{R}^{d} ; \mathbb{C}^{m}\right) \rightarrow L_{2}\left(\mathbb{R}^{d} ; \mathbb{C}^{n}\right)}
$$

can be estimated by a quantity depending only on $\|g\|_{L_{\infty}},\left\|g^{-1}\right\|_{L_{\infty}}, \alpha_{0}, m, n, d$, and $\Omega$. Finally, using (7.16) with $\ell=d / 2-1$ and also (7.19), (7.20), we get

$$
\left\|\Lambda b(\mathbf{D})(I-\Pi) \mathcal{E}^{0}(\tau)\right\|_{L_{2}\left(\mathbb{R}^{d} ; \mathbb{C}^{n}\right) \rightarrow L_{2}\left(\mathbb{R}^{d} ; \mathbb{C}^{n}\right)} \leq \lambda \tau^{-1}, \quad \tau \geq 1
$$

where

$$
\begin{aligned}
\lambda:=c_{0}^{-3 / 2} c_{1}^{1 / 2} \widehat{c} m^{1 / 2}|\Omega|^{1 / 2}\|f\|_{L_{\infty}}^{2}\left\|f^{-1}\right\|_{L_{\infty}}^{2}\left(1+r_{0}^{-2}\right)^{1 / 2}\left(1+1 / 4 r_{0}^{2}\right)^{1 / 2}, & d \leq 4, \\
\lambda:=c_{0}^{-1 / 2-d / 4} c_{1}^{1 / 2} \widehat{c} m^{1 / 2}|\Omega|^{1 / 2} C(d)\|f\|_{L_{\infty}}^{2}\left\|f^{-1}\right\|_{L_{\infty}}^{d / 2} & \\
\times\left(1+r_{0}^{-2}\right)^{d / 4-1 / 2}\left(1+1 / 4 r_{0}^{2}\right)^{1 / 2}, & d>4 .
\end{aligned}
$$

Here $\widehat{c}$ is the constant occurring in (7.19),

$$
C(d):=\max _{x \geq 0}\left\{x^{d / 4} e^{-x}\right\} .
$$

Thus, in (7.3) we can replace $\Pi$ by $I$ in the case where $\tau \geq 1$. As a result, using (7.3), (7.5), (7.9), (7.10), (7.13), (7.14), (7.15), and (7.21), we arrive at the following theorem.

Theorem 7.1. For $\tau \geq 1$ we have

$$
\begin{aligned}
f e^{-\mathcal{A} \tau} f^{*}= & \mathcal{E}^{0}(\tau)+\Lambda_{Q} b(\mathbf{D}) \mathcal{E}^{0}(\tau)+\mathcal{E}^{0}(\tau)\left(\Lambda_{Q} b(\mathbf{D})\right)^{*} \\
& -\int_{0}^{\tau} \mathcal{E}^{0}(\tau-\widetilde{\tau}) b(\mathbf{D})^{*} L_{Q}(\mathbf{D}) b(\mathbf{D}) \mathcal{E}^{0}(\widetilde{\tau}) d \widetilde{\tau}+\mathcal{J}_{0}(\tau),
\end{aligned}
$$

where

$$
\left\|\mathcal{J}_{0}(\tau)\right\|_{L_{2}\left(\mathbb{R}^{d} ; \mathbb{C}^{n}\right) \rightarrow L_{2}\left(\mathbb{R}^{d} ; \mathbb{C}^{n}\right)} \leq \mathcal{C}_{0} \tau^{-1}, \quad \mathcal{C}_{0}:=\mathcal{D}_{4}+\eta^{(1)}+\eta^{(3)}+2\left(\lambda+\lambda_{0}\right) .
$$

For $\tau \geq 0$ we have

$$
\begin{aligned}
f e^{-\mathcal{A} \tau} f^{*}= & \mathcal{E}^{0}(\tau)+\Lambda_{Q} b(\mathbf{D}) \Pi \mathcal{E}^{0}(\tau)+\mathcal{E}^{0}(\tau)\left(\Lambda_{Q} b(\mathbf{D}) \Pi\right)^{*} \\
& -\int_{0}^{\tau} \mathcal{E}^{0}(\tau-\widetilde{\tau}) b(\mathbf{D})^{*} L_{Q}(\mathbf{D}) b(\mathbf{D}) \Pi \mathcal{E}^{0}(\widetilde{\tau}) d \widetilde{\tau}+\mathcal{J}_{1}(\tau)
\end{aligned}
$$

Here

$$
\left\|\mathcal{J}_{1}(\tau)\right\|_{L_{2}\left(\mathbb{R}^{d} ; \mathbb{C}^{n}\right) \rightarrow L_{2}\left(\mathbb{R}^{d} ; \mathbb{C}^{n}\right)} \leq \mathcal{C}_{1}(1+\tau)^{-1}, \quad \mathcal{C}_{1}:=\mathcal{D}_{5}+\eta^{(2)} .
$$

The constants $\mathcal{D}_{4}, \mathcal{D}_{5}, \eta^{(1)}, \eta^{(2)}, \eta^{(3)}, \lambda_{0}$, and $\lambda$ are defined in (6.7), (7.11), (7.12), (7.15), and (7.22).

If some additional conditions are fulfilled, the "elimination" of the operator $\Pi$ in all terms of (7.3) is possible not only for $\tau \geq 1$, but also for all $\tau>0$. As noticed above, if $d \leq 4$, then $\Lambda$ is a continuous operator from $H^{1}\left(\mathbb{R}^{d} ; \mathbb{C}^{m}\right)$ to $L_{2}\left(\mathbb{R}^{d} ; \mathbb{C}^{n}\right)$; i.e., in this case we can put $\ell=1$. Obviously, to obtain formulas (7.16), now we do not need the condition $\tau \geq 1$. Thus, the following statement is true.

Proposition 7.2. If $d \leq 4$, then the representation (7.23) is valid for $\tau>0$ with estimate (7.24), where $\lambda$ is the constant defined by the first line in (7.22).

Now we consider the case where $\Lambda \in L_{\infty}\left(\mathbb{R}^{d}\right)$. The estimation of the first term on the right in (7.14) is similar to that of the second term, and again we do not need the condition $\tau \geq 1$. 
E. S. VASILEVSKAYA

Proposition 7.3. If $\Lambda \in L_{\infty}\left(\mathbb{R}^{d}\right)$, then the representation (7.23) is valid for all $\tau>0$ with estimate (7.24), where

$$
\lambda=c_{0}^{-1} \alpha_{1}^{1 / 2} r_{0}^{-1}\|f\|_{L_{\infty}}^{2}\left\|f^{-1}\right\|_{L_{\infty}}^{2}\|\Lambda\|_{L_{\infty}} .
$$

Consider the case where $g^{0}=\bar{g}$ (see (5.4)). In this case the right-hand sides of (7.3), (7.23), and (7.25) simplify appreciably. By Remark 5.1 in [6], for $g^{0}=\bar{g}$ we have $\Lambda_{Q}=0$, so that $\widehat{Z}_{Q}(\boldsymbol{\theta})=0, \hat{N}_{Q}(\boldsymbol{\theta})=0$. In this case, (7.3), (7.5), (7.9), and (7.10) imply the following assertion.

Proposition 7.4. If $g^{0}=\bar{g}$, then

$$
\begin{array}{ll}
\left\|f e^{-\mathcal{A} \tau} f^{*}-\mathcal{E}^{0}(\tau)\right\|_{L_{2}\left(\mathbb{R}^{d} ; \mathbb{C}^{n}\right) \rightarrow L_{2}\left(\mathbb{R}^{d} ; \mathbb{C}^{n}\right)} \leq\left(\mathcal{D}_{4}+\eta^{(1)}\right) \tau^{-1}, & \\
\left\|f e^{-\mathcal{A} \tau} f^{*}-\mathcal{E}^{0}(\tau)\right\|_{L_{2}\left(\mathbb{R}^{d} ; \mathbb{C}^{n}\right) \rightarrow L_{2}\left(\mathbb{R}^{d} ; \mathbb{C}^{n}\right)} \leq\left(\mathcal{D}_{5}+\eta^{(2)}\right)(1+\tau)^{-1}, & \tau \geq 0 .
\end{array}
$$

7.3. The case where $f=\mathbf{1}_{n}$. In the case where $f=\mathbf{1}_{n}$, the statement of Theorem 7.1 simplifies. In formulas (7.23) and (7.25), $f$ and $f_{0}$ are replaced by $\mathbf{1}_{n}, \Lambda_{Q}$ is replaced by $\Lambda, \mathcal{A}^{0}$ is replaced by $\hat{\mathcal{A}}^{0}$ (see $(7.1)$ ), and $L_{Q}(\mathbf{D})$ is replaced by the operator $L(\mathbf{D})$ with the symbol

$$
L(\boldsymbol{\theta}):=|\Omega|^{-1} \int_{\Omega}\left(\Lambda(\mathbf{x})^{*} b(\boldsymbol{\theta})^{*} \widetilde{g}(\mathbf{x})+\widetilde{g}(\mathbf{x})^{*} b(\boldsymbol{\theta}) \Lambda(\mathbf{x})\right) d \mathbf{x} .
$$

We formulate the corresponding result.

Theorem 7.5. For $\tau \geq 1$ we have

$$
\begin{aligned}
e^{-\hat{\mathcal{A}} \tau}= & e^{-\hat{\mathcal{A}}^{0} \tau}+\Lambda b(\mathbf{D}) e^{-\hat{\mathcal{A}}^{0} \tau}+e^{-\hat{\mathcal{A}}^{0} \tau}(\Lambda b(\mathbf{D}))^{*} \\
& -\int_{0}^{\tau} e^{-\hat{\mathcal{A}}^{0}(\tau-\widetilde{\tau})} b(\mathbf{D})^{*} L(\mathbf{D}) b(\mathbf{D}) e^{-\hat{\mathcal{A}}^{0} \tilde{\tau}} d \widetilde{\tau}+\widehat{\mathcal{J}}_{0}(\tau),
\end{aligned}
$$

where

For $\tau \geq 0$ we have

$$
\left\|\widehat{\mathcal{J}}_{0}(\tau)\right\|_{L_{2}\left(\mathbb{R}^{d} ; \mathbb{C}^{n}\right) \rightarrow L_{2}\left(\mathbb{R}^{d} ; \mathbb{C}^{n}\right)} \leq \widehat{\mathcal{C}}_{0} \tau^{-1}
$$

$$
\begin{aligned}
e^{-\hat{\mathcal{A}} \tau}= & e^{-\hat{\mathcal{A}}^{0} \tau}+\Lambda b(\mathbf{D}) \Pi e^{-\hat{\mathcal{A}}^{0} \tau}+e^{-\hat{\mathcal{A}}^{0} \tau}(\Lambda b(\mathbf{D}) \Pi)^{*} \\
& -\int_{0}^{\tau} e^{-\hat{\mathcal{A}}^{0}(\tau-\widetilde{\tau})} b(\mathbf{D})^{*} L(\mathbf{D}) b(\mathbf{D}) \Pi e^{-\hat{\mathcal{A}}^{0} \tau} d \widetilde{\tau}+\widehat{\mathcal{J}}_{1}(\tau) .
\end{aligned}
$$

Here

$$
\left\|\widehat{\mathcal{J}}_{1}(\tau)\right\|_{L_{2}\left(\mathbb{R}^{d} ; \mathbb{C}^{n}\right) \rightarrow L_{2}\left(\mathbb{R}^{d} ; \mathbb{C}^{n}\right)} \leq \widehat{\mathcal{C}}_{1}(1+\tau)^{-1} .
$$

The constants $\widehat{\mathcal{C}_{0}}$ and $\widehat{\mathcal{C}}_{1}$ can be found by putting $\|f\|_{L_{\infty}}=1,\left\|f^{-1}\right\|_{L_{\infty}}=1$ in the expressions for $\mathcal{C}_{0}$ and $\mathcal{C}_{1}$.

Aside from Propositions 7.2-7.4, some simplifications are possible in the case where $f=\mathbf{1}_{n}$ for $g^{0}=\underline{g}$. In [6, Proposition 4.5(2)] it was shown that if $g^{0}=\underline{g}$, then $L(\boldsymbol{\theta})=0$, whence $N(\boldsymbol{\theta})=0$. We shall also need [6, Proposition 6.9], where it was shown that for $g^{0}=\underline{g}$ we have $\Lambda \in L_{\infty}$. Using Proposition 7.3, we arrive at the following statement.

Proposition 7.6. Suppose $g^{0}=\underline{g}$. Then

$$
\begin{array}{r}
\left\|e^{-\hat{\mathcal{A}} \tau}-e^{-\hat{\mathcal{A}}^{0} \tau}-\Lambda b(\mathbf{D}) e^{-\hat{\mathcal{A}}^{0} \tau}-e^{-\hat{\mathcal{A}}^{0} \tau}(\Lambda b(\mathbf{D}))^{*}\right\|_{L_{2}\left(\mathbb{R}^{d}\right) \rightarrow L_{2}\left(\mathbb{R}^{d}\right)} \leq \hat{C}_{0} \tau^{-1}, \\
\tau>0, \\
\left\|e^{-\hat{\mathcal{A}} \tau}-e^{-\hat{\mathcal{A}}^{0} \tau}-\Lambda b(\mathbf{D}) \Pi e^{-\hat{\mathcal{A}}^{0} \tau}-e^{-\hat{\mathcal{A}}^{0} \tau}(\Lambda b(\mathbf{D}) \Pi)^{*}\right\|_{L_{2}\left(\mathbb{R}^{d}\right) \rightarrow L_{2}\left(\mathbb{R}^{d}\right)} \leq \widehat{C}_{1}(1+\tau)^{-1}, \\
\tau \geq 0 .
\end{array}
$$


Here

$$
\widehat{C}_{0}:=\widehat{\mathcal{D}}_{4}+\widehat{\eta}^{(1)}+2 \hat{\lambda}, \quad \widehat{C}_{1}:=\widehat{\mathcal{D}}_{5}+\widehat{\eta}^{(2)},
$$

and the constants $\hat{\mathcal{D}}_{4}, \hat{\mathcal{D}}_{5}, \hat{\eta}^{(1)}, \hat{\eta}^{(2)}$, and $\hat{\lambda}$ can be found by putting $\|f\|_{L_{\infty}}=1$, $\left\|f^{-1}\right\|_{L_{\infty}}=1$ in $(6.7),(7.11)$, and (7.27).

7.4. Example: the operator $\mathcal{A}=f^{*} \mathbf{D}^{*} g \mathbf{D} f$. In $L_{2}\left(\mathbb{R}^{d}\right)$ with $d \geq 1$, consider the operator

$$
\mathcal{A}=f(\mathbf{x})^{*} \mathbf{D}^{*} g(\mathbf{x}) \mathbf{D} f(\mathbf{x})=-f(\mathbf{x})^{*} \operatorname{div} g(\mathbf{x}) \nabla f(\mathbf{x}),
$$

where $g(\mathbf{x})$ is a $\Gamma$-periodic $(d \times d)$-matrix-valued function with real entries, and $f(\mathbf{x})$ is a $\Gamma$-periodic (in general, complex-valued) function. It is assumed that

$$
g(\mathbf{x})>0, \quad g, g^{-1}, f, f^{-1} \in L_{\infty} .
$$

In this case $n=1, m=d$, and $b(\boldsymbol{\xi})=\boldsymbol{\xi}$. Clearly, conditions (4.1) and (4.2) are satisfied and $\alpha_{0}=\alpha_{1}=1$. The function $Q(\mathbf{x})$, defined in (4.22), turns out to be $\Gamma$-periodic and real-valued.

We shall follow the logic of [6. Subsection 10.1]. Let $v_{j} \in \widetilde{H}^{1}(\Omega), j=1, \ldots, d$, be the solutions of equations (5.1), understood in the weak sense. Since these solutions are now purely imaginary, it is convenient to introduce the functions $\Phi_{j}(\mathbf{x})=-i v_{j}(\mathbf{x})$, $j=1, \ldots, d$, which solve the equations

$$
\operatorname{div} g(\mathbf{x})\left(\nabla \Phi_{j}(\mathbf{x})+\mathbf{e}_{j}\right)=0, \quad \int_{\Omega} \Phi_{j}(\mathbf{x}) d \mathbf{x}=0 .
$$

The functions $\Phi_{j}(\mathbf{x})$ are real-valued, and the matrix $\Lambda(\mathbf{x})$ introduced in Subsection 5.1 can be rewritten as

$$
\Lambda(\mathbf{x})=i\left(\Phi_{1}(\mathbf{x}), \ldots, \Phi_{d}(\mathbf{x})\right) .
$$

The matrix $\widetilde{g}(\mathbf{x})$ (see $(5.2))$ is a real-valued $(d \times d)$-matrix whose columns have the following form: $g(\mathbf{x})\left(\nabla \Phi_{j}(\mathbf{x})+\mathbf{e}_{j}\right), j=1, \ldots, d$. As before, the effective matrix $g^{0}$ is defined by formula (5.3).

Our goal is to apply Theorem 7.1 to the operator $\mathcal{A}=f^{*} \mathbf{D}^{*} g \mathbf{D} f$. First, observe that $\Lambda_{Q}^{0}$ (see (5.7)) is a constant matrix-arrow with purely imaginary elements. Therefore, $\Lambda_{Q}^{0} \mathbf{D} \mathcal{E}^{0}(\tau)+\mathcal{E}^{0}(\tau)\left(\Lambda_{Q}^{0} \mathbf{D}\right)^{*}=0$. Furthermore, in this case,

$$
\Lambda b(\mathbf{D})=\sum_{j=1}^{d} \Phi_{j}(\mathbf{x}) \partial_{j} .
$$

By Corollary 5.4 in [6], for the operator $\mathcal{A}=f^{*} \mathbf{D}^{*} g \mathbf{D} f$ with a real-valued matrix $g(\mathbf{x})$ we have $\hat{N}_{Q}(\boldsymbol{\theta})=0$. This implies that in (7.23) the fourth term on the right is equal to zero. Finally, from elliptic theory (see [13, Chapter III, Theorem 13.1]) it follows that in the case in question we have $\Lambda \in L_{\infty}$. Recalling that now $\alpha_{0}=\alpha_{1}=1$, from Proposition 7.3 and formulas (7.23), (7.24), (4.5), we conclude that the operator $\mathcal{A}=f^{*} \mathbf{D}^{*} g \mathbf{D} f$, which satisfies the conditions formulated above, is represented in the form

$$
\begin{aligned}
& f e^{-\mathcal{A} \tau} f^{*}=\mathcal{E}^{0}(\tau)+\sum_{j=1}^{d} \Phi_{j}(\mathbf{x}) \partial_{j} \mathcal{E}^{0}(\tau)-\mathcal{E}^{0}(\tau) \sum_{j=1}^{d} \partial_{j} \Phi_{j}(\mathbf{x})+\mathcal{J}_{0}(\tau), \quad \tau>0 \\
& \left\|\mathcal{J}_{0}(\tau)\right\|_{L_{2}\left(\mathbb{R}^{d}\right) \rightarrow L_{2}\left(\mathbb{R}^{d}\right)} \leq C_{0}^{\prime} \tau^{-1} \\
& C_{0}^{\prime}:=\mathcal{D}_{4}+\eta^{(1)}+2 r_{0}^{-1}\left\|g^{-1}\right\|_{L_{\infty}}\|f\|_{L_{\infty}}^{2}\left\|f^{-1}\right\|_{L_{\infty}}^{2}\|\Lambda\|_{L_{\infty}}
\end{aligned}
$$

where $\mathcal{D}_{4}, \eta^{(1)}$ are the constants defined in (6.7) and (7.11).

We note that, as was mentioned in [6], there exist examples where $N_{Q}(\boldsymbol{\theta})$ differs from zero not only for the scalar elliptic operator $f^{*} \mathbf{D}^{*} g(\mathbf{x}) \mathbf{D} f$ with complex-valued matrix 
$g(\mathbf{x})$, but also for matrix operators $f^{*} b(\mathbf{D})^{*} g(\mathbf{x}) b(\mathbf{D}) f$ even in the case of real-valued matrices $b(\boldsymbol{\theta})$ and $g(\mathbf{x})$. Thus, in general, the third term of the corrector is nontrivial.

\section{Chapter 3. HOMOGENIZATION OF A PERIODIC PARABOLIC CAUCHY PROBLEM}

§8. Approximation of the "Bordered" operator exponential $f^{\varepsilon} e^{-\mathcal{A}_{\varepsilon} \tau}\left(f^{\varepsilon}\right)^{*}$

8.1. Homogenization with corrector for the operator $f^{\varepsilon} e^{-\mathcal{A}_{\varepsilon} \tau}\left(f^{\varepsilon}\right)^{*}$. Consider an operator family in $\mathfrak{G}:=L_{2}\left(\mathbb{R}^{d} ; \mathbb{C}^{n}\right)$ defined by the expression

$$
\mathcal{A}_{\varepsilon}=\left(f^{\varepsilon}(\mathbf{x})\right)^{*} b(\mathbf{D})^{*} g^{\varepsilon}(\mathbf{x}) b(\mathbf{D}) f^{\varepsilon}(\mathbf{x}), \quad \varepsilon>0,
$$

where $g^{\varepsilon}(\mathbf{x}):=g\left(\varepsilon^{-1} \mathbf{x}\right), f^{\varepsilon}(\mathbf{x}):=f\left(\varepsilon^{-1} \mathbf{x}\right)$. The coefficients of the operator $\mathcal{A}_{\varepsilon}$ rapidly oscillate as $\varepsilon \rightarrow 0$. We consider the behavior of the "bordered" operator exponential $f^{\varepsilon} e^{-\mathcal{A}_{\varepsilon} \tau}\left(f^{\varepsilon}\right)^{*}$ in the limit as $\varepsilon \rightarrow 0$. The leading term of the required approximation was obtained in [15]. We formulate the result of Theorem 7.3 in [15]: for $\tau \geq 0$ and $\varepsilon>0$ we have

$$
\left\|f^{\varepsilon} e^{-\mathcal{A}_{\varepsilon} \tau}\left(f^{\varepsilon}\right)^{*}-\mathcal{E}^{0}(\tau)\right\|_{\mathfrak{G} \rightarrow \mathfrak{G}} \leq \mathfrak{C}\|f\|_{L_{\infty}}^{2} \varepsilon\left(\tau+\varepsilon^{2}\right)^{-1 / 2},
$$

where $\mathfrak{C}$ is the constant defined in [15, (6.11)] and depending only on $\alpha_{0}, \alpha_{1}, r_{0},\|g\|_{L_{\infty}}$, $\left\|g^{-1}\right\|_{L_{\infty}},\|f\|_{L_{\infty}}$, and $\left\|f^{-1}\right\|_{L_{\infty}}$.

Now we obtain an approximation with corrector for the operator $f^{\varepsilon} e^{-\mathcal{A}_{\varepsilon} \tau}\left(f^{\varepsilon}\right)^{*}$. We introduce a scaling operator $T_{\varepsilon}$ that acts by the formula

$$
\left(T_{\varepsilon} \mathbf{u}\right)(\mathbf{x})=\varepsilon^{d / 2} \mathbf{u}(\varepsilon \mathbf{x}), \quad \mathbf{x} \in \mathbb{R}^{d} .
$$

This operator is unitary in $\mathfrak{G}$. Since $\mathcal{A}_{\varepsilon}=\varepsilon^{-2} T_{\varepsilon}^{*} \mathcal{A} T_{\varepsilon}$, we have

$$
\begin{aligned}
f^{\varepsilon} e^{-\mathcal{A}_{\varepsilon} \tau}\left(f^{\varepsilon}\right)^{*} & =T_{\varepsilon}^{*} f e^{-\mathcal{A} \tau \varepsilon^{-2}} f^{*} T_{\varepsilon}, & T_{\varepsilon}^{*} \Lambda_{Q} b(\mathbf{D}) T_{\varepsilon} & =\varepsilon \Lambda_{Q}^{\varepsilon} b(\mathbf{D}), \\
\mathcal{E}^{0}(\tau) & =T_{\varepsilon}^{*} \mathcal{E}^{0}\left(\tau \varepsilon^{-2}\right) T_{\varepsilon}, & T_{\varepsilon}^{*} b(\mathbf{D})^{*} L_{Q}(\mathbf{D}) b(\mathbf{D}) T_{\varepsilon} & =\varepsilon^{3} b(\mathbf{D})^{*} L_{Q}(\mathbf{D}) b(\mathbf{D}),
\end{aligned}
$$

where $\Lambda_{Q}^{\varepsilon}(\mathbf{x}):=\Lambda_{Q}\left(\varepsilon^{-1} \mathbf{x}\right)$. We also denote $\Pi_{\varepsilon}:=T_{\varepsilon}^{*} \Pi T_{\varepsilon}$. Then, by (7.6),

$$
\left(\Pi_{\varepsilon} \mathbf{u}\right)(\mathbf{x})=(2 \pi)^{-d / 2} \int_{\widetilde{\Omega} / \varepsilon} e^{i\langle\mathbf{k}, \mathbf{x}\rangle}(\mathcal{F} \mathbf{u})(\mathbf{k}) d \mathbf{k} .
$$

We replace $\tau$ by $\tau \varepsilon^{-2}$ in formulas (7.23) and (7.25), and then apply the operator $T_{\varepsilon}^{*}$ from the left and the operator $T_{\varepsilon}$ from the right. As a result, we arrive at the following theorem.

Theorem 8.1. For $\varepsilon^{2} \leq \tau, \varepsilon>0$, we have

$$
\begin{aligned}
f^{\varepsilon} e^{-\mathcal{A}_{\varepsilon} \tau}\left(f^{\varepsilon}\right)^{*}= & \mathcal{E}^{0}(\tau)+\varepsilon\left(\Lambda_{Q}^{\varepsilon} b(\mathbf{D}) \mathcal{E}^{0}(\tau)+\mathcal{E}^{0}(\tau)\left(\Lambda_{Q}^{\varepsilon} b(\mathbf{D})\right)^{*}\right. \\
& \left.\quad-\int_{0}^{\tau} \mathcal{E}^{0}(\tau-\widetilde{\tau}) b(\mathbf{D})^{*} L_{Q}(\mathbf{D}) b(\mathbf{D}) \mathcal{E}^{0}(\widetilde{\tau}) d \widetilde{\tau}\right) \\
& +Y_{0}(\tau ; \varepsilon),
\end{aligned}
$$

where

$$
\left\|Y_{0}(\tau ; \varepsilon)\right\|_{\mathfrak{G} \rightarrow \mathfrak{G}} \leq \mathcal{C}_{0} \tau^{-1} \varepsilon^{2},
$$

and $\mathcal{C}_{0}$ is the constant defined in (7.24). 
For arbitrary $\varepsilon>0$ and $\tau \geq 0$, we have

$$
\begin{aligned}
f^{\varepsilon} e^{-\mathcal{A}_{\varepsilon} \tau}\left(f^{\varepsilon}\right)^{*}= & \mathcal{E}^{0}(\tau)+\varepsilon\left(\Lambda_{Q}^{\varepsilon} b(\mathbf{D}) \Pi_{\varepsilon} \mathcal{E}^{0}(\tau)+\mathcal{E}^{0}(\tau)\left(\Lambda_{Q}^{\varepsilon} b(\mathbf{D}) \Pi_{\varepsilon}\right)^{*}\right. \\
& \left.-\int_{0}^{\tau} \mathcal{E}^{0}(\tau-\widetilde{\tau}) b(\mathbf{D})^{*} L_{Q}(\mathbf{D}) b(\mathbf{D}) \Pi_{\varepsilon} \mathcal{E}^{0}(\widetilde{\tau}) d \widetilde{\tau}\right) \\
& +Y_{1}(\tau ; \varepsilon) .
\end{aligned}
$$

Here

$$
\left\|Y_{1}(\tau ; \varepsilon)\right\|_{\mathfrak{G} \rightarrow \mathfrak{G}} \leq \mathcal{C}_{1}\left(\varepsilon^{2}+\tau\right)^{-1} \varepsilon^{2},
$$

where $\mathcal{C}_{1}$ is the constant defined in (7.26).

8.2. The case where $f=\mathbf{1}_{n}$. We state Theorem 8.1 in the special case where $f=\mathbf{1}_{n}$.

Theorem 8.2. For $\varepsilon^{2} \leq \tau, \varepsilon>0$, we have

$$
\begin{aligned}
e^{-\hat{\mathcal{A}}_{\varepsilon} \tau}=e^{-\hat{\mathcal{A}}^{0} \tau}+\varepsilon( & \Lambda^{\varepsilon} b(\mathbf{D}) e^{-\hat{\mathcal{A}}^{0} \tau}+e^{-\hat{\mathcal{A}}^{0} \tau}\left(\Lambda^{\varepsilon} b(\mathbf{D})\right)^{*} \\
& \left.-\int_{0}^{\tau} e^{-\hat{\mathcal{A}}^{0}(\tau-\widetilde{\tau})} b(\mathbf{D})^{*} L(\mathbf{D}) b(\mathbf{D}) e^{-\hat{\mathcal{A}}^{0} \tilde{\tau}} d \tilde{\tau}\right)+\hat{Y}_{0}(\tau ; \varepsilon),
\end{aligned}
$$

where

$$
\left\|\widehat{Y}_{0}(\tau ; \varepsilon)\right\|_{\mathfrak{G} \rightarrow \mathfrak{G}} \leq \widehat{\mathcal{C}}_{0} \tau^{-1} \varepsilon^{2} .
$$

For arbitrary $\varepsilon>0$ and $\tau \geq 0$, we have

$$
\begin{aligned}
e^{-\hat{\mathcal{A}}_{\varepsilon} \tau}=e^{-\hat{\mathcal{A}}^{0} \tau}+\varepsilon( & \Lambda^{\varepsilon} b(\mathbf{D}) \Pi_{\varepsilon} e^{-\hat{\mathcal{A}}^{0} \tau}+e^{-\hat{\mathcal{A}}^{0} \tau}\left(\Lambda^{\varepsilon} b(\mathbf{D}) \Pi_{\varepsilon}\right)^{*} \\
& \left.-\int_{0}^{\tau} e^{-\hat{\mathcal{A}}^{0}(\tau-\widetilde{\tau})} b(\mathbf{D})^{*} L(\mathbf{D}) b(\mathbf{D}) \Pi_{\varepsilon} e^{-\hat{\mathcal{A}}^{0} \widetilde{\tau}} d \tilde{\tau}\right)+\hat{Y}_{1}(\tau ; \varepsilon) .
\end{aligned}
$$

Here

$$
\left\|\widehat{Y}_{1}(\tau ; \varepsilon)\right\|_{\mathfrak{G} \rightarrow \mathfrak{G}} \leq \widehat{\mathcal{C}}_{1}\left(\varepsilon^{2}+\tau\right)^{-1} \varepsilon^{2},
$$

and $\widehat{\mathcal{C}}_{0}, \widehat{\mathcal{C}}_{1}$ are the constants that can be found by putting $\|f\|_{L_{\infty}}=\left\|f^{-1}\right\|_{L_{\infty}}=1$ in (7.24) and (7.26).

8.3. Example: the operator $\mathcal{A}=f^{*} \mathbf{D}^{*} g \mathbf{D} f$. Consider the example described in Subsection 7.4. The operator $\mathcal{A}=f^{*} \mathbf{D}^{*} g \mathbf{D} f$ is assumed to satisfy the conditions formulated above. Consider also the operator

$$
\mathcal{A}_{\varepsilon}=\left(f^{\varepsilon}\right)^{*} \mathbf{D}^{*} g^{\varepsilon} \mathbf{D} f^{\varepsilon}
$$

with rapidly oscillating coefficients. Applying the scaling transformation to (7.28), we obtain

$$
\begin{gathered}
f^{\varepsilon} e^{-\mathcal{A}_{\varepsilon} \tau}\left(f^{\varepsilon}\right)^{*}=\mathcal{E}^{0}(\tau)+\varepsilon\left(\sum_{j=1}^{d} \Phi_{j}^{\varepsilon}(\mathbf{x}) \partial_{j} \mathcal{E}^{0}(\tau)-\mathcal{E}^{0}(\tau) \sum_{j=1}^{d} \partial_{j} \Phi_{j}^{\varepsilon}(\mathbf{x})\right)+Y_{0}(\tau ; \varepsilon), \\
\left\|Y_{0}(\tau ; \varepsilon)\right\|_{L_{2}\left(\mathbb{R}^{d}\right) \rightarrow L_{2}\left(\mathbb{R}^{d}\right)} \leq C_{0}^{\prime} \tau^{-1} \varepsilon^{2}, \quad \varepsilon>0, \quad \tau>0 .
\end{gathered}
$$

The constant $C_{0}^{\prime}$ is defined in (7.29). 
8.4. Comparison with the elliptic problem. In [6, Subsection 9.1], an approximation as $\varepsilon \rightarrow 0$ for the resolvent $\left(\mathcal{A}_{\varepsilon}+I\right)^{-1}$ in the $L_{2}\left(\mathbb{R}^{d} ; \mathbb{C}^{n}\right)$-operator norm was obtained with error estimate of order of $\varepsilon^{2}$. To be precise, the following formula [6, (9.7)] was established:

$$
\left(\mathcal{A}_{\varepsilon}+I\right)^{-1}=\left(f^{\varepsilon}\right)^{-1}\left(\widehat{\mathcal{R}}_{Q}^{0}+\varepsilon K_{Q}(\varepsilon)\right)\left(\left(f^{\varepsilon}\right)^{*}\right)^{-1}+\varepsilon^{2} \mathcal{Y}^{\prime}(\varepsilon),
$$

where $\hat{\mathcal{R}}_{Q}^{0}:=\left(\hat{\mathcal{A}}^{0}+\bar{Q}\right)^{-1}$ and the operator $K_{Q}(\varepsilon)$, called, in accordance with [6], a corrector, looks like this:

$$
K_{Q}(\varepsilon)=\Lambda_{Q}^{\varepsilon} b(\mathbf{D}) \Pi_{\varepsilon} \hat{\mathcal{R}}_{Q}^{0}+\widehat{\mathcal{R}}_{Q}^{0}\left(\Lambda_{Q}^{\varepsilon} b(\mathbf{D}) \Pi_{\varepsilon}\right)^{*}-\widehat{\mathcal{R}}_{Q}^{0} b(\mathbf{D})^{*} L_{Q}(\mathbf{D}) b(\mathbf{D}) \widehat{\mathcal{R}}_{Q}^{0} .
$$

Here

$$
\left\|\mathcal{Y}^{\prime}(\varepsilon)\right\|_{\mathfrak{G} \rightarrow \mathfrak{G}} \leq \mathcal{C}_{1}^{\prime}\left\|f^{-1}\right\|_{L_{\infty}}^{2}
$$

and the constant $\mathcal{C}_{1}^{\prime}$ depends only on $\|g\|_{L_{\infty}},\left\|g^{-1}\right\|_{L_{\infty}},\|Q\|_{L_{\infty}},\left\|Q^{-1}\right\|_{L_{\infty}}, \alpha_{0}, \alpha_{1}, m$, and the parameters of the lattice. The operator that stands in the term involving the first degree of $\varepsilon$ in (8.2) or (8.3) will also be called a corrector. The correctors in (8.2) and (8.3) will be denoted as follows (respectively):

$$
\begin{aligned}
\mathcal{K}(\varepsilon, \tau)= & \Lambda_{Q}^{\varepsilon} b(\mathbf{D}) \mathcal{E}^{0}(\tau)+\mathcal{E}^{0}(\tau)\left(\Lambda_{Q}^{\varepsilon} b(\mathbf{D})\right)^{*} \\
& -\int_{0}^{\tau} \mathcal{E}^{0}(\tau-\widetilde{\tau}) b(\mathbf{D})^{*} L_{Q}(\mathbf{D}) b(\mathbf{D}) \mathcal{E}^{0}(\widetilde{\tau}) d \widetilde{\tau}, \\
\mathcal{K}^{\prime}(\varepsilon, \tau)= & \Lambda_{Q}^{\varepsilon} b(\mathbf{D}) \Pi_{\varepsilon} \mathcal{E}^{0}(\tau)+\mathcal{E}^{0}(\tau)\left(\Lambda_{Q}^{\varepsilon} b(\mathbf{D}) \Pi_{\varepsilon}\right)^{*} \\
& -\int_{0}^{\tau} \mathcal{E}^{0}(\tau-\widetilde{\tau}) b(\mathbf{D})^{*} L_{Q}(\mathbf{D}) b(\mathbf{D}) \Pi_{\varepsilon} \mathcal{E}^{0}(\widetilde{\tau}) d \widetilde{\tau} .
\end{aligned}
$$

It is clear that, in the case of a parabolic problem, as well as for an elliptic problem, the corrector contains three terms, two of them being adjoint to each other and containing a rapidly oscillating term $\Lambda_{Q}^{\varepsilon}$. The third term of the corrector for the parabolic problem is similar to the corresponding term for the elliptic problem. In the case of the corrector (8.8) the situation is absolutely similar: in this case the term in question does not depend on $\varepsilon$ and involves no variable (depending on $\mathbf{x}$ ) coefficients. The third term of the corrector (8.9) depends on $\varepsilon$ because of the presence of $\Pi_{\varepsilon}$.

Now we discuss the possibility of elimination of the smoothing operator $\Pi_{\varepsilon}$ from the "elliptic" and "parabolic" correctors. In the elliptic case the third term of the corrector does not contain $\Pi_{\varepsilon}$, whereas from the first and the second terms the operator $\Pi_{\varepsilon}$ can be eliminated only in special cases. For example, we can eliminate it in the case where $d \leq 4$ or in the case of a scalar elliptic operator $f^{*} \mathbf{D}^{*} g(\mathbf{x}) \mathbf{D} f$ with a real-valued matrix $g(\mathbf{x})$ for any $d$. As was shown above, in the parabolic case, if the condition $\varepsilon^{2} \leq \tau$ is fulfilled, then the operator $\Pi_{\varepsilon}$ can be completely eliminated from all terms of the corrector. This is an essential difference between the results in the parabolic and elliptic cases. On the other hand, if we do not impose the restriction $\varepsilon^{2} \leq \tau$ and let $\varepsilon$ and $\tau$ be arbitrary $(\varepsilon>0$, $\tau \geq 0$ ), we must retain the operator $\Pi_{\varepsilon}$ in all terms of the "parabolic" corrector (8.9). It cannot be eliminated even from the third term.

8.5. Derivation of "elliptic" results from "parabolic" ones. We mention the following fact. A result concerning homogenization with corrector for the elliptic problem can be derived from that for the parabolic problem by using the following representation:

$$
\left(\mathcal{A}_{\varepsilon}+I\right)^{-1}=\int_{0}^{\infty} e^{-\mathcal{A}_{\varepsilon} \tau} e^{-\tau} d \tau .
$$


However, the estimate in this case is of order $\varepsilon^{2}|\ln \varepsilon|$ instead of the estimate of order $\varepsilon^{2}$ obtained in 6]. We show this in more detail. We use Theorem 8.1. Then

$$
\begin{aligned}
f^{\varepsilon}\left(\int_{0}^{\infty} e^{-\mathcal{A}_{\varepsilon} \tau} e^{-\tau} d \tau\right)\left(f^{\varepsilon}\right)^{*}=\int_{0}^{\infty} \mathcal{E}^{0}(\tau) e^{-\tau} d \tau \\
+\varepsilon\left(\Lambda_{Q}^{\varepsilon} b(\mathbf{D}) \Pi_{\varepsilon} \int_{0}^{\infty} \mathcal{E}^{0}(\tau) e^{-\tau} d \tau+\left(\int_{0}^{\infty} \mathcal{E}^{0}(\tau) e^{-\tau} d \tau\right)\left(\Lambda_{Q}^{\varepsilon} b(\mathbf{D}) \Pi_{\varepsilon}\right)^{*}\right. \\
\left.\quad-\int_{0}^{\infty} e^{-\tau} d \tau \int_{0}^{\tau} \mathcal{E}^{0}(\tau-\widetilde{\tau}) b(\mathbf{D})^{*} L_{Q}(\mathbf{D}) b(\mathbf{D}) \Pi_{\varepsilon} \mathcal{E}^{0}(\widetilde{\tau}) d \widetilde{\tau}\right)+\tilde{Y}_{1}(\varepsilon)
\end{aligned}
$$

where

$$
\tilde{Y}_{1}(\varepsilon):=\int_{0}^{\infty} Y_{1}(\tau ; \varepsilon) e^{-\tau} d \tau
$$

Recall the notation $\hat{\mathcal{R}}_{Q}^{0}=\left(\widehat{\mathcal{A}}^{0}+\bar{Q}\right)^{-1}$. Using (4.24) and (7.1), we rewrite $\widehat{\mathcal{R}}_{Q}^{0}$ as $\hat{\mathcal{R}}_{Q}^{0}=$ $f_{0}\left(f_{0} \widehat{\mathcal{A}}^{0} f_{0}+I\right)^{-1} f_{0}=f_{0}\left(\mathcal{A}^{0}+I\right)^{-1} f_{0}$. Then, by $(7.4)$,

$$
\widehat{\mathcal{R}}_{Q}^{0}=\int_{0}^{\infty} \mathcal{E}^{0}(\tau) e^{-\tau} d \tau
$$

We rewrite (8.11), taking (7.4), (8.10), and (8.13) into account:

$$
f^{\varepsilon}\left(\mathcal{A}_{\varepsilon}+I\right)^{-1}\left(f^{\varepsilon}\right)^{*}=\widehat{\mathcal{R}}_{Q}^{0}+\varepsilon\left(\Lambda_{Q}^{\varepsilon} b(\mathbf{D}) \Pi_{\varepsilon} \widehat{\mathcal{R}}_{Q}^{0}+\widehat{\mathcal{R}}_{Q}^{0}\left(\Lambda_{Q}^{\varepsilon} b(\mathbf{D}) \Pi_{\varepsilon}\right)^{*}-\mathcal{L}(\varepsilon)\right)+\tilde{Y}_{1}(\varepsilon),
$$

where

$$
\mathcal{L}(\varepsilon):=\int_{0}^{\infty} f_{0} e^{-\mathcal{A}^{0} \tau} e^{-\tau} d \tau \int_{0}^{\tau} e^{\mathcal{A}^{0} \tilde{\tau}} f_{0} b(\mathbf{D})^{*} L_{Q}(\mathbf{D}) b(\mathbf{D}) \Pi_{\varepsilon} f_{0} e^{-\mathcal{A}^{0} \tilde{\tau}} f_{0} d \tilde{\tau} .
$$

In order to transform expression (8.15), we note that

$$
e^{-\mathcal{A}^{0} \tau} e^{-\tau}=-\frac{d}{d \tau}\left(\mathcal{A}^{0}+I\right)^{-1} e^{-\left(\mathcal{A}^{0}+I\right) \tau} .
$$

Thus, integration by parts yields

$$
\begin{aligned}
\mathcal{L}(\varepsilon)= & -\left.\left(f_{0}\left(\mathcal{A}^{0}+I\right)^{-1} e^{-\left(\mathcal{A}^{0}+I\right) \tau} \int_{0}^{\tau} e^{\mathcal{A}^{0} \tilde{\tau}} f_{0} b(\mathbf{D})^{*} L_{Q}(\mathbf{D}) b(\mathbf{D}) \Pi_{\varepsilon} f_{0} e^{-\mathcal{A}^{0} \tilde{\tau}} f_{0} d \widetilde{\tau}\right)\right|_{0} ^{\infty} \\
& +\int_{0}^{\infty} f_{0}\left(\mathcal{A}^{0}+I\right)^{-1} e^{-\left(\mathcal{A}^{0}+I\right) \tau} e^{\mathcal{A}^{0} \tau} f_{0} b(\mathbf{D})^{*} L_{Q}(\mathbf{D}) b(\mathbf{D}) \Pi_{\varepsilon} f_{0} e^{-\mathcal{A}^{0} \tau} f_{0} d \tau .
\end{aligned}
$$

Obviously, substitution of the lower limit in the first term gives zero. We show that substitution of the upper limit also gives zero for any $\varepsilon>0$. We estimate the norm of the operator

$$
\mathcal{L}_{1}(\varepsilon, \tau):=e^{-\tau} f_{0}\left(\mathcal{A}^{0}+I\right)^{-1} \int_{0}^{\tau} e^{-\mathcal{A}^{0}(\tau-\widetilde{\tau})} f_{0} b(\mathbf{D})^{*} L_{Q}(\mathbf{D}) b(\mathbf{D}) \Pi_{\varepsilon} f_{0} e^{-\mathcal{A}^{0} \tilde{\tau}} f_{0} d \widetilde{\tau} .
$$

Clearly,

$$
\left\|\mathcal{L}_{1}(\varepsilon, \tau)\right\| \leq \tau e^{-\tau}\left\|f_{0}\right\|_{L_{\infty}}^{4}\left\|b(\mathbf{D})^{*} L_{Q}(\mathbf{D}) b(\mathbf{D}) \Pi_{\varepsilon}\right\| .
$$

Thus, for any $\varepsilon>0$ we see that $\left\|\mathcal{L}_{1}(\varepsilon, \tau)\right\| \rightarrow 0$ as $\tau \rightarrow \infty$. Now the expression for $\mathcal{L}(\varepsilon)$ has the following form:

$$
\begin{aligned}
\mathcal{L}(\varepsilon) & =\int_{0}^{\infty} f_{0}\left(\mathcal{A}^{0}+I\right)^{-1} e^{-\tau} f_{0} b(\mathbf{D})^{*} L_{Q}(\mathbf{D}) b(\mathbf{D}) \Pi_{\varepsilon} f_{0} e^{-\mathcal{A}^{0} \tau} f_{0} d \tau \\
& =\widehat{\mathcal{R}}_{Q}^{0} b(\mathbf{D})^{*} L_{Q}(\mathbf{D}) b(\mathbf{D}) \Pi_{\varepsilon} \hat{\mathcal{R}}_{Q}^{0},
\end{aligned}
$$


E. S. VASILEVSKAYA

which coincides with the third term of the "elliptic" corrector (before the "elimination" of $\Pi$ ). To estimate the operator $\widetilde{Y}_{1}(\varepsilon)$ in (8.12), we use (8.4), obtaining

$$
\left\|\tilde{Y}_{1}(\varepsilon)\right\| \leq \mathcal{C}_{1} \varepsilon^{2} \int_{0}^{\infty} \frac{e^{-\tau}}{\tau+\varepsilon^{2}} d \tau
$$

Transforming the integral on the right-hand side, it is easy to show that, for $\varepsilon>0$,

$$
\left\|\tilde{Y}_{1}(\varepsilon)\right\| \leq \mathcal{C}_{1} \varepsilon^{2}(2|\ln \varepsilon|+\check{c}), \quad \check{c}=2 \ln 2+\int_{1}^{\infty} e^{-\tau} \ln \tau d \tau .
$$

Using formula (7.12) in [6], namely,

$$
\left\|\widehat{\mathcal{R}}_{Q}^{0}(\varepsilon) b(\mathbf{D})^{*} L_{Q}(\mathbf{D}) b(\mathbf{D})(I-\Pi) \hat{\mathcal{R}}_{Q}^{0}(\varepsilon)\right\| \leq 2^{1 / 2} \alpha_{0}^{-5 / 2} \alpha_{1}^{3 / 2}\|g\|_{L_{\infty}}^{3 / 2}\left\|g^{-1}\right\|_{L_{\infty}}^{5 / 2} r_{0}^{-2},
$$

where $\hat{\mathcal{R}}_{Q}^{0}(\varepsilon):=\left(\hat{\mathcal{A}}^{0}+\varepsilon^{2} \bar{Q}\right)$, and applying the scaling transformation to it, we see that

$$
\left\|\hat{\mathcal{R}}_{Q}^{0} b(\mathbf{D})^{*} L_{Q}(\mathbf{D}) b(\mathbf{D})\left(I-\Pi_{\varepsilon}\right) \widehat{\mathcal{R}}_{Q}^{0}\right\| \leq 2^{1 / 2} \alpha_{0}^{-5 / 2} \alpha_{1}^{3 / 2}\|g\|_{L_{\infty}}^{3 / 2}\left\|g^{-1}\right\|_{L_{\infty}}^{5 / 2} r_{0}^{-2} \varepsilon .
$$

Finally, from (8.14), (8.16), (8.17), and (8.18), we conclude that

$$
\left(\mathcal{A}_{\varepsilon}+I\right)^{-1}=\left(f^{\varepsilon}\right)^{-1}\left(\widehat{\mathcal{R}}_{Q}^{0}+\varepsilon K_{Q}(\varepsilon)\right)\left(\left(f^{\varepsilon}\right)^{*}\right)^{-1}+\varepsilon^{2} \mathcal{Y}^{\prime \prime}(\varepsilon),
$$

where $K_{Q}(\varepsilon)$ is defined in (8.6). However, in contrast to (8.5), where the operator $\mathcal{Y}^{\prime}(\varepsilon)$ admits the estimate of order 1 (see (8.7)), now we have a term containing $|\ln \varepsilon|$ :

$$
\left\|\mathcal{Y}^{\prime \prime}(\varepsilon)\right\| \leq\left((2|\ln \varepsilon|+\check{c}) \mathcal{C}_{1}+2^{1 / 2} c_{0}^{-5 / 2} c_{1}^{3 / 2} r_{0}^{-2}\right)\left\|f^{-1}\right\|_{L_{\infty}}^{2} .
$$

8.6. Weak limit of the corrector. Let us find the weak operator limit of the correctors (8.8), (8.9). The third term in (8.8) does not depend on $\varepsilon$. Since the weak limit of the operators $\Pi_{\varepsilon}$ is equal to $I$, and the operator $\int_{0}^{\tau} \mathcal{E}^{0}(\tau-\widetilde{\tau}) b(\mathbf{D})^{*} L_{Q}(\mathbf{D}) b(\mathbf{D}) \mathcal{E}^{0}(\widetilde{\tau}) d \widetilde{\tau}$ is bounded for fixed $\tau>0$, the weak limit of the third term of the corrector (8.9) is equal to the third term in (8.8). Consider the first term of the corrector. We shall use the following fact, which follows from "average value property" (see, e.g., Lemma 8.6 in [ 6 ): for a $\Gamma$-periodic function $Y$ of class $L_{2, \text { loc }}\left(\mathbb{R}^{d}\right)$, the weak $\left(L_{2}\left(\mathbb{R}^{d}\right) \rightarrow L_{2}\left(\mathbb{R}^{d}\right)\right)$-limit of the operators $\left[Y^{\varepsilon}\right] \Pi_{\varepsilon}$ can be found by the formula

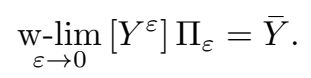

Since the operator $b(\mathbf{D}) \mathcal{E}^{0}(\tau)$ is bounded for $\tau>0$ and $\bar{\Lambda}=0$, we have

$$
\underset{\varepsilon \rightarrow 0}{\mathrm{w}-\lim _{\varepsilon \rightarrow 0}}\left(\Lambda_{Q}^{\varepsilon} b(\mathbf{D}) \mathcal{E}^{0}(\tau) \Pi_{\varepsilon}\right)=\Lambda_{Q}^{0} b(\mathbf{D}) \mathcal{E}^{0}(\tau), \quad \tau>0
$$

(see (5.7)). Thus, for the corrector (8.9), for $\tau>0$ we have

$$
\begin{aligned}
\underset{\varepsilon \rightarrow 0}{\mathrm{w}-\lim _{\varepsilon \rightarrow 0}} \mathcal{K}^{\prime}(\varepsilon, \tau)= & \Lambda_{Q}^{0} b(\mathbf{D}) \mathcal{E}^{0}(\tau)+\left(\Lambda_{Q}^{0} b(\mathbf{D}) \mathcal{E}^{0}(\tau)\right)^{*} \\
& -\int_{0}^{\tau} \mathcal{E}^{0}(\tau-\widetilde{\tau}) b(\mathbf{D})^{*} L_{Q}(\mathbf{D}) b(\mathbf{D}) \mathcal{E}^{0}(\widetilde{\tau}) d \widetilde{\tau}
\end{aligned}
$$

For the corrector (8.8) the weak limit is the same, because

$$
\left\|\Lambda_{Q}^{\varepsilon} b(\mathbf{D}) \mathcal{E}^{0}(\tau)\left(I-\Pi_{\varepsilon}\right)\right\|_{L_{2}\left(\mathbb{R}^{d}\right) \rightarrow L_{2}\left(\mathbb{R}^{d}\right)} \longrightarrow 0, \quad \varepsilon \rightarrow 0, \quad \tau>0 .
$$

This can easily be shown by applying the scaling transformation to (7.15), (7.21). Thus,

$$
\begin{aligned}
\underset{\varepsilon \rightarrow 0}{\mathrm{w}-\lim _{i} \mathcal{K}(\varepsilon, \tau)=} & \Lambda_{Q}^{0} b(\mathbf{D}) \mathcal{E}^{0}(\tau)+\left(\Lambda_{Q}^{0} b(\mathbf{D}) \mathcal{E}^{0}(\tau)\right)^{*} \\
& -\int_{0}^{\tau} \mathcal{E}^{0}(\tau-\widetilde{\tau}) b(\mathbf{D})^{*} L_{Q}(\mathbf{D}) b(\mathbf{D}) \mathcal{E}^{0}(\widetilde{\tau}) d \widetilde{\tau} .
\end{aligned}
$$


Note that in the case where $Q=\mathbf{1}_{n}$ (for $\hat{\mathcal{A}}_{\varepsilon}$ ), the first two terms on the right-hand side are equal to zero, and the weak limit of the corrector (8.8) coincides with its third term.

\section{§9. Application to a homogeneous Cauchy problem}

9.1. Application to a homogeneous Cauchy problem. Consider the homogeneous Cauchy problem

$$
Q^{\varepsilon}(\mathbf{x}) \frac{\partial \mathbf{u}_{\varepsilon}(\mathbf{x}, \tau)}{\partial \tau}=-b(\mathbf{D})^{*} g^{\varepsilon}(\mathbf{x}) b(\mathbf{D}) \mathbf{u}_{\varepsilon}(\mathbf{x}, \tau), \quad Q^{\varepsilon}(\mathbf{x}) \mathbf{u}_{\varepsilon}(\mathbf{x}, 0)=\phi(\mathbf{x}),
$$

where $Q(\mathbf{x})$ is a $\Gamma$-periodic $(n \times n)$-matrix-valued bounded and uniformly positive function, and $\phi \in L_{2}\left(\mathbb{R}^{d} ; \mathbb{C}^{n}\right)$. Then the solution $\mathbf{u}_{\varepsilon}$ of $(9.1)$ converges as $\varepsilon \rightarrow 0$ to the solution $\mathbf{u}_{0}$ of the "homogenized" problem

$$
\bar{Q} \frac{\partial \mathbf{u}_{0}(\mathbf{x}, \tau)}{\partial \tau}=-b(\mathbf{D})^{*} g^{0} b(\mathbf{D}) \mathbf{u}_{0}(\mathbf{x}, \tau), \quad \bar{Q} \mathbf{u}_{0}(\mathbf{x}, 0)=\phi(\mathbf{x}) .
$$

More precisely, we write the matrix $Q(\mathbf{x})$ in a factorized form: $Q(\mathbf{x})^{-1}=f(\mathbf{x}) f(\mathbf{x})^{*}$. Then, it is easy to verify that

$$
\mathbf{u}_{\varepsilon}(\cdot, \tau)=f^{\varepsilon} e^{-\mathcal{A}_{\varepsilon} \tau}\left(f^{\varepsilon}\right)^{*} \phi, \quad \mathbf{u}_{0}(\cdot, \tau)=f_{0} e^{-\mathcal{A}^{0} \tau} f_{0} \phi, \quad f_{0}=(\bar{Q})^{-1 / 2} .
$$

Using Theorem 8.1, we arrive at the following result.

Theorem 9.1. Let $\mathbf{u}_{\varepsilon}(\mathbf{x}, \tau)$ be the solution of problem (9.1), and let $\mathbf{u}_{0}(\mathbf{x}, \tau)$ be the solution of problem (9.2). Then for $\varepsilon^{2} \leq \tau, \varepsilon>0$, we have

$$
\begin{gathered}
\mathbf{u}_{\varepsilon}(\cdot, \tau)=\mathbf{u}_{0}(\cdot, \tau)+\varepsilon\left(\Lambda_{Q}^{\varepsilon} b(\mathbf{D}) \mathbf{u}_{0}(\cdot, \tau)+\mathcal{E}^{0}(\tau)\left(\Lambda_{Q}^{\varepsilon} b(\mathbf{D})\right)^{*} \boldsymbol{\phi}\right. \\
\left.-\int_{0}^{\tau} \mathcal{E}^{0}(\tau-\widetilde{\tau}) b(\mathbf{D})^{*} L(\mathbf{D}) b(\mathbf{D}) \mathbf{u}_{0}(\cdot, \widetilde{\tau}) d \widetilde{\tau}\right)+Y_{0}(\tau ; \varepsilon) \boldsymbol{\phi} \\
\left\|Y_{0}(\tau ; \varepsilon) \phi\right\|_{\mathfrak{G}} \leq \mathcal{C}_{0} \tau^{-1} \varepsilon^{2}\|\boldsymbol{\phi}\|_{\mathfrak{G}}
\end{gathered}
$$

For $\varepsilon>0, \tau \geq 0$, we have

$$
\begin{gathered}
\mathbf{u}_{\varepsilon}(\cdot, \tau)=\mathbf{u}_{0}(\cdot, \tau)+\varepsilon\left(\Lambda_{Q}^{\varepsilon} b(\mathbf{D}) \Pi_{\varepsilon} \mathbf{u}_{0}(\cdot, \tau)+\mathcal{E}^{0}(\tau)\left(\Lambda_{Q}^{\varepsilon} b(\mathbf{D}) \Pi_{\varepsilon}\right)^{*} \phi\right. \\
\left.-\int_{0}^{\tau} \mathcal{E}^{0}(\tau-\widetilde{\tau}) b(\mathbf{D})^{*} L(\mathbf{D}) b(\mathbf{D}) \Pi_{\varepsilon} \mathbf{u}_{0}(\cdot, \widetilde{\tau}) d \widetilde{\tau}\right)+Y_{1}(\tau ; \varepsilon) \boldsymbol{\phi}, \\
\left\|Y_{1}(\tau ; \varepsilon) \boldsymbol{\phi}\right\|_{\mathfrak{G}} \leq \mathcal{C}_{1}\left(\varepsilon^{2}+\tau\right)^{-1} \varepsilon^{2}\|\phi\|_{\mathfrak{G}}
\end{gathered}
$$

Here $\mathcal{C}_{0}$ and $\mathcal{C}_{1}$ are the constants defined in (7.24), (7.26).

9.2. The case where $f=\mathbf{1}_{n}$. Consider the homogeneous Cauchy problem

$$
\frac{\partial \widehat{\mathbf{u}}_{\varepsilon}(\mathbf{x}, \tau)}{\partial \tau}=-\widehat{\mathcal{A}}_{\varepsilon} \widehat{\mathbf{u}}_{\varepsilon}(\mathbf{x}, \tau), \quad \widehat{\mathbf{u}}_{\varepsilon}(\mathbf{x}, 0)=\phi(\mathbf{x})
$$

where

$$
\widehat{\mathcal{A}}_{\varepsilon}=b(\mathbf{D})^{*} g^{\varepsilon}(\mathbf{x}) b(\mathbf{D}), \quad \varepsilon>0,
$$

and the corresponding "homogenized" problem

$$
\frac{\partial \widehat{\mathbf{u}}_{0}(\mathbf{x}, \tau)}{\partial \tau}=-\widehat{\mathcal{A}}^{0} \widehat{\mathbf{u}}_{0}(\mathbf{x}, \tau), \quad \widehat{\mathbf{u}}_{0}(\mathbf{x}, 0)=\phi(\mathbf{x})
$$

where $\hat{\mathcal{A}}^{0}$ is the effective operator (5.6). We formulate Theorem 9.1 in the special case where $f=\mathbf{1}_{n}$. 
Theorem 9.2. Let $\widehat{\mathbf{u}}_{\varepsilon}(\mathbf{x}, \tau)$ be the solution of problem (9.3), and let $\widehat{\mathbf{u}}_{0}(\mathbf{x}, \tau)$ be the solution of problem (9.4). Then for $\varepsilon^{2} \leq \tau, \varepsilon>0$, we have

$$
\begin{gathered}
\widehat{\mathbf{u}}_{\varepsilon}(\cdot, \tau)=\widehat{\mathbf{u}}_{0}(\cdot, \tau)+\varepsilon\left(\Lambda^{\varepsilon} b(\mathbf{D}) \widehat{\mathbf{u}}_{0}(\cdot, \tau)+e^{-\hat{\mathcal{A}}^{0} \tau}\left(\Lambda^{\varepsilon} b(\mathbf{D})\right)^{*} \phi\right. \\
\left.-\int_{0}^{\tau} e^{-\hat{\mathcal{A}}^{0}(\tau-\widetilde{\tau})} b(\mathbf{D})^{*} L(\mathbf{D}) b(\mathbf{D}) \widehat{\mathbf{u}}_{0}(\cdot, \widetilde{\tau}) d \widetilde{\tau}\right)+\widehat{Y}_{0}(\tau ; \varepsilon) \boldsymbol{\phi}, \\
\left\|\widehat{Y}_{0}(\tau ; \varepsilon) \phi\right\|_{\mathfrak{G}} \leq \widehat{\mathcal{C}}_{0} \tau^{-1} \varepsilon^{2}\|\phi\|_{\mathfrak{G}} .
\end{gathered}
$$

For $\varepsilon>0, \tau \geq 0$, we have

$$
\begin{gathered}
\widehat{\mathbf{u}}_{\varepsilon}(\cdot, \tau)=\widehat{\mathbf{u}}_{0}(\cdot, \tau)+\varepsilon\left(\Lambda^{\varepsilon} b(\mathbf{D}) \Pi_{\varepsilon} \widehat{\mathbf{u}}_{0}(\cdot, \tau)+e^{-\hat{\mathcal{A}}^{0} \tau}\left(\Lambda^{\varepsilon} b(\mathbf{D}) \Pi_{\varepsilon}\right)^{*} \phi\right. \\
\left.-\int_{0}^{\tau} e^{-\hat{\mathcal{A}}^{0}(\tau-\widetilde{\tau})} b(\mathbf{D})^{*} L(\mathbf{D}) b(\mathbf{D}) \Pi_{\varepsilon} \widehat{\mathbf{u}}_{0}(\cdot, \widetilde{\tau}) d \widetilde{\tau}\right)+\widehat{Y}_{1}(\tau ; \varepsilon) \boldsymbol{\phi}, \\
\left\|\widehat{Y}_{1}(\tau ; \varepsilon) \phi\right\|_{\mathfrak{G}} \leq \widehat{\mathcal{C}}_{1}\left(\varepsilon^{2}+\tau\right)^{-1} \varepsilon^{2}\|\boldsymbol{\phi}\|_{\mathfrak{G}} .
\end{gathered}
$$

Here $\widehat{\mathcal{C}}_{0}$ and $\widehat{\mathcal{C}}_{1}$ are the same as in Theorem 8.2 .

\section{§10. Applichtion to a nonhomogeneous Cauchy problem}

10.1. Application to a nonhomogeneous Cauchy problem. Consider the nonhomogeneous Cauchy problem

$$
Q^{\varepsilon}(\mathbf{x}) \frac{\partial \mathbf{u}_{\varepsilon}(\mathbf{x}, \tau)}{\partial \tau}=-b(\mathbf{D})^{*} g^{\varepsilon} b(\mathbf{D}) \mathbf{u}_{\varepsilon}(\mathbf{x}, \tau)+\mathbf{F}(\mathbf{x}, \tau), \quad Q^{\varepsilon}(\mathbf{x}) \mathbf{u}_{\varepsilon}(\mathbf{x}, 0)=\phi(\mathbf{x}),
$$

where $\mathbf{x} \in \mathbb{R}^{d}$ and $\tau \in(0, T), T>0$. Suppose that $\phi \in \mathfrak{G}$ and $\mathbf{F} \in \mathcal{H}_{p}$ for some $1<p \leq \infty$, where we use the notation $\mathcal{H}_{p}:=L_{p}\left((0, T) ; L_{2}\left(\mathbb{R}^{d} ; \mathbb{C}^{n}\right)\right)$. Let $\mathbf{u}_{0}$ be the solution of the "homogenized" problem

$$
\bar{Q} \frac{\partial \mathbf{u}_{0}(\mathbf{x}, \tau)}{\partial \tau}=-b(\mathbf{D})^{*} g^{0} b(\mathbf{D}) \mathbf{u}_{0}(\mathbf{x}, \tau)+\mathbf{F}(\mathbf{x}, \tau), \quad \bar{Q} \mathbf{u}_{0}(\mathbf{x}, 0)=\phi(\mathbf{x}) .
$$

In [15, Subsection 7.4], Theorem 7.4 was formulated, in which the convergence of the solutions $\mathbf{u}_{\varepsilon}(\cdot, \tau)$ to $\mathbf{u}_{0}(\cdot, \tau)$ in the operator norm in $\mathfrak{G}$ as $\varepsilon \rightarrow 0$, for each $\tau \in(0, T)$, was claimed with the estimate

$$
\left\|\mathbf{u}_{\varepsilon}(\cdot, \tau)-\mathbf{u}_{0}(\cdot, \tau)\right\|_{\mathfrak{G}} \leq \mathfrak{C} \varepsilon\left\|Q^{-1}\right\|_{L_{\infty}}\left(\left(\tau+\varepsilon^{2}\right)^{-1 / 2}\|\phi\|_{\mathfrak{G}}+\theta(p, \varepsilon, \tau)\|\mathbf{F}\|_{\mathcal{H}_{p}}\right),
$$

where $\varepsilon \leq 1, \mathfrak{C}$ is the same as in $(8.1)$, and

$$
\theta(p, \varepsilon, \tau)= \begin{cases}(\tau+1)^{1 / 2-1 / p}\left(1-p^{\prime} / 2\right)^{-1 / p^{\prime}}, & 2<p \leq \infty, \\ (\ln (\tau+1)+2|\ln \varepsilon|)^{1 / 2}, & p=2, \\ \varepsilon^{1-2 / p}\left(p^{\prime} / 2-1\right)^{-1 / p^{\prime}}, & 1<p<2 .\end{cases}
$$

Now we apply Theorem 8.1 in order to improve this approximation by invoking a corrector. We shall use the representations [15, (7.7), (7.9)]:

$$
\begin{aligned}
& \mathbf{u}_{\varepsilon}=f^{\varepsilon} e^{-\mathcal{A}_{\varepsilon} \tau}\left(f^{\varepsilon}\right)^{*} \phi+\int_{0}^{\tau} f^{\varepsilon} e^{-\mathcal{A}_{\varepsilon}(\tau-\widetilde{\tau})}\left(f^{\varepsilon}\right)^{*} \mathbf{F}(\cdot, \widetilde{\tau}) d \widetilde{\tau} \\
& \mathbf{u}_{0}=f_{0} e^{-\mathcal{A}^{0} \tau} f_{0} \phi+\int_{0}^{\tau} f_{0} e^{-\mathcal{A}_{0}(\tau-\widetilde{\tau})} f_{0} \mathbf{F}(\cdot, \widetilde{\tau}) d \widetilde{\tau}
\end{aligned}
$$


With the help of (8.3) and (8.4), the first term on the right in (10.5) can be rewritten in the form

$$
\begin{aligned}
f^{\varepsilon} e^{-\mathcal{A}_{\varepsilon} \tau}\left(f^{\varepsilon}\right)^{*} \phi= & \mathcal{E}^{0}(\tau) \phi+\varepsilon\left(\Lambda_{Q}^{\varepsilon} b(\mathbf{D}) \Pi_{\varepsilon} \mathcal{E}^{0}(\tau) \phi+\mathcal{E}^{0}(\tau)\left(\Lambda_{Q}^{\varepsilon} b(\mathbf{D}) \Pi_{\varepsilon}\right)^{*} \phi\right) \\
& -\varepsilon \int_{0}^{\tau} \mathcal{E}^{0}(\tau-\widetilde{\tau}) b(\mathbf{D})^{*} L_{Q}(\mathbf{D}) b(\mathbf{D}) \Pi_{\varepsilon} \mathcal{E}^{0}(\widetilde{\tau}) \phi d \widetilde{\tau}+Y_{1}(\tau ; \varepsilon) \boldsymbol{\phi}
\end{aligned}
$$

where

$$
\left\|Y_{1}(\tau ; \varepsilon) \phi\right\|_{\mathfrak{G}} \leq \mathcal{C}_{1} \varepsilon^{2}\left(\tau+\varepsilon^{2}\right)^{-1}\|\phi\|_{\mathfrak{G}}
$$

This implies that, for any $0<\varepsilon \leq 1, \tau \in(0, T)$, and $1<p \leq \infty$, formulas (10.7), (10.8) give a sharper estimate of the first term on the right in (10.5), compared to the corresponding estimate without corrector (see (10.3)).

Consider the second term on the right in (10.5), assuming that $2<p \leq \infty$. We rewrite this term as a sum of two terms, assuming that $\tau>\varepsilon^{2}$ :

$$
\begin{aligned}
\int_{0}^{\tau} f^{\varepsilon} e^{-\mathcal{A}_{\varepsilon}(\tau-\widetilde{\tau})}\left(f^{\varepsilon}\right)^{*} \mathbf{F}(\cdot, \widetilde{\tau}) d \widetilde{\tau}= & \int_{0}^{\tau-\varepsilon^{2}} f^{\varepsilon} e^{-\mathcal{A}_{\varepsilon}(\tau-\widetilde{\tau})}\left(f^{\varepsilon}\right)^{*} \mathbf{F}(\cdot, \widetilde{\tau}) d \widetilde{\tau} \\
& +\int_{\tau-\varepsilon^{2}}^{\tau} f^{\varepsilon} e^{-\mathcal{A}_{\varepsilon}(\tau-\widetilde{\tau})}\left(f^{\varepsilon}\right)^{*} \mathbf{F}(\cdot, \widetilde{\tau}) d \widetilde{\tau}
\end{aligned}
$$

By (8.3), we have

$$
\begin{aligned}
& \int_{0}^{\tau-\varepsilon^{2}} f^{\varepsilon} e^{-\mathcal{A}_{\varepsilon}(\tau-\widetilde{\tau})}\left(f^{\varepsilon}\right)^{*} \mathbf{F}(\cdot, \widetilde{\tau}) d \widetilde{\tau}=\int_{0}^{\tau-\varepsilon^{2}} \mathcal{E}^{0}(\tau-\widetilde{\tau}) \mathbf{F}(\cdot, \widetilde{\tau}) d \widetilde{\tau} \\
& +\varepsilon \int_{0}^{\tau-\varepsilon^{2}}\left(\Lambda_{Q}^{\varepsilon} b(\mathbf{D}) \Pi_{\varepsilon} \mathcal{E}^{0}(\tau-\widetilde{\tau}) \mathbf{F}(\cdot, \widetilde{\tau})+\mathcal{E}^{0}(\tau-\widetilde{\tau})\left(\Lambda_{Q}^{\varepsilon} b(\mathbf{D}) \Pi_{\varepsilon}\right)^{*} \mathbf{F}(\cdot, \widetilde{\tau})\right) d \widetilde{\tau} \\
& \quad-\varepsilon \int_{0}^{\tau-\varepsilon^{2}} d \widetilde{\tau} \int_{0}^{\tau-\widetilde{\tau}} \mathcal{E}^{0}\left(\tau-\widetilde{\tau}-\tau^{\prime}\right) b(\mathbf{D})^{*} L_{Q}(\mathbf{D}) b(\mathbf{D}) \Pi_{\varepsilon} \mathcal{E}^{0}\left(\tau^{\prime}\right) \mathbf{F}(\cdot, \widetilde{\tau}) d \tau^{\prime}+\phi_{1}
\end{aligned}
$$

where

$$
\phi_{1}:=\int_{0}^{\tau-\varepsilon^{2}} Y_{1}(\tau-\widetilde{\tau} ; \varepsilon) \mathbf{F}(\cdot, \widetilde{\tau}) d \widetilde{\tau} .
$$

We estimate $\left\|\phi_{1}\right\|_{\mathfrak{G}}$ with the help of (8.4). Recalling that $\mathbf{F} \in \mathcal{H}_{p}$ and introducing the index $p^{\prime}=p /(p-1)$, from (10.11) for $2<p<\infty$ we conclude that

$$
\begin{aligned}
\phi_{1} \|_{\mathfrak{G}} & \leq\|\mathbf{F}\|_{\mathcal{H}_{p}}\left(\int_{0}^{\tau-\varepsilon^{2}}\left\|Y_{1}(\tau-\widetilde{\tau} ; \varepsilon)\right\|^{p^{\prime}} d \widetilde{\tau}\right)^{1 / p^{\prime}} \\
& \leq\|\mathbf{F}\|_{\mathcal{H}_{p}}\left(\int_{\varepsilon^{2}}^{\tau}\left(\mathcal{C}_{1} \varepsilon^{2}\right)^{p^{\prime}}\left(\varepsilon^{2}+y\right)^{-p^{\prime}} d y\right)^{1 / p^{\prime}} \\
& =\|\mathbf{F}\|_{\mathcal{H}_{p}} \mathcal{C}_{1}\left(p^{\prime}-1\right)^{-1 / p^{\prime}} \varepsilon^{2 / p^{\prime}}\left(2^{1-p^{\prime}}-\left(\tau \varepsilon^{-2}+1\right)^{1-p^{\prime}}\right)^{1 / p^{\prime}} \\
& \leq\|\mathbf{F}\|_{\mathcal{H}_{p}} \mathcal{C}_{1} 2^{-1 / p}\left(p^{\prime}-1\right)^{-1 / p^{\prime}} \varepsilon^{2 / p^{\prime}}
\end{aligned}
$$

In the case where $\mathbf{F} \in \mathcal{H}_{\infty}$, we have

$$
\begin{aligned}
\left\|\phi_{1}\right\|_{\mathfrak{G}} & \leq\|\mathbf{F}\|_{\mathcal{H}_{\infty}} \mathcal{C}_{1} \varepsilon^{2} \int_{\varepsilon^{2}}^{\tau}\left(\varepsilon^{2}+y\right)^{-1} d y=\|\mathbf{F}\|_{\mathcal{H}_{\infty}} \mathcal{C}_{1} \varepsilon^{2}\left(\ln \left(\tau+\varepsilon^{2}\right)+2|\ln \varepsilon|\right) \\
& \leq\|\mathbf{F}\|_{\mathcal{H}_{\infty}} \mathcal{C}_{1} \varepsilon^{2}(\ln (\tau+1)+2|\ln \varepsilon|), \quad \varepsilon \leq 1
\end{aligned}
$$


We want to show that, in the second and third terms on the right-hand side of (10.10), the upper limit of integration can be replaced by $\tau$. We use the relation $\mathbf{F} \in \mathcal{H}_{p}$ once again. The case where $p=\infty$ is also covered: take $p^{\prime}=1$. Using (7.7), we obtain

$$
\begin{aligned}
& \left\|\int_{\tau-\varepsilon^{2}}^{\tau} \Lambda_{Q}^{\varepsilon} b(\mathbf{D}) \Pi_{\varepsilon} \mathcal{E}^{0}(\tau-\widetilde{\tau}) \mathbf{F}(\cdot, \tilde{\tau}) d \widetilde{\tau}\right\|_{\mathfrak{G}} \\
& \quad \leq\|\mathbf{F}\|_{\mathcal{H}_{p}}\left(\int_{0}^{\varepsilon^{2}}\left\|\Lambda_{Q}^{\varepsilon} b(\mathbf{D}) \Pi_{\varepsilon} \mathcal{E}^{0}(y)\right\|_{\mathfrak{G} \rightarrow \mathfrak{G}}^{p^{\prime}} d y\right)^{1 / p^{\prime}} \\
& \quad=\|\mathbf{F}\|_{\mathcal{H}_{p}}\left(\int_{0}^{\varepsilon^{2}}\left\|\varepsilon^{-1} T_{\varepsilon}^{*} \Lambda_{Q} b(\mathbf{D}) \Pi \mathcal{E}^{0}\left(y \varepsilon^{-2}\right) T_{\varepsilon}\right\|_{\mathfrak{G} \rightarrow \mathfrak{G}}^{p^{\prime}} d y\right)^{1 / p^{\prime}} \\
& \leq\|\mathbf{F}\|_{\mathcal{H}_{p}}\left\|\Lambda_{Q} b(\mathbf{D}) \Pi\right\|\|f\|_{L_{\infty}}^{2} \varepsilon^{2 / p^{\prime}-1} .
\end{aligned}
$$

The norm of the operator $\Lambda_{Q} b(\mathbf{D}) \Pi$ can be estimated by using (5.10) and the unitary equivalence

$$
\Lambda_{Q} b(\mathbf{D}) \Pi=\mathcal{U}^{-1}\left(\int_{\widetilde{\Omega}} \oplus \hat{Z}_{Q}(\mathbf{k}) d \mathbf{k}\right) \mathcal{U}
$$

(see (5.8)). We have

$$
\begin{aligned}
\left\|\Lambda_{Q} b(\mathbf{D}) \Pi\right\|_{\mathfrak{G} \rightarrow \mathfrak{G}} & =\sup _{\mathbf{k} \in \widetilde{\Omega}}\left\|\hat{Z}_{Q}(\mathbf{k})\right\|_{L_{2}(\Omega) \rightarrow L_{2}(\Omega)} \\
& \leq r_{1} \sup _{|\boldsymbol{\theta}|=1}\left\|\hat{Z}_{Q}(\boldsymbol{\theta})\right\|_{L_{2}(\Omega) \rightarrow L_{2}(\Omega)} \leq \mu_{1}, \\
\mu_{1} & :=2^{-1 / 2} c_{0}^{-1 / 2} c_{1}^{1 / 2} r_{0}^{-1} r_{1}\|Q\|_{L_{\infty}}\left\|Q^{-1}\right\|_{L_{\infty}} .
\end{aligned}
$$

Here we have used the fact that $|\mathbf{k}| \leq r_{1}$ for $\mathbf{k} \in \widetilde{\Omega}$; also, we transformed the constant in (5.10), with the help of (4.5) and (4.22). In a similar way,

$$
\begin{aligned}
& \left\|\int_{\tau-\varepsilon^{2}}^{\tau} d \widetilde{\tau} \int_{0}^{\tau-\tau} \mathcal{E}^{0}\left(\tau-\widetilde{\tau}-\tau^{\prime}\right) b(\mathbf{D})^{*} L_{Q}(\mathbf{D}) b(\mathbf{D}) \Pi_{\varepsilon} \mathcal{E}^{0}\left(\tau^{\prime}\right) \mathbf{F}(\cdot, \widetilde{\tau}) d \tau^{\prime}\right\|_{\mathfrak{G}} \\
& \leq\|\mathbf{F}\|_{\mathcal{H}_{p}}\left(\int_{0}^{\varepsilon^{2}}\left\|\int_{0}^{y} \mathcal{E}^{0}\left(y-\tau^{\prime}\right) b(\mathbf{D})^{*} L_{Q}(\mathbf{D}) b(\mathbf{D}) \Pi_{\varepsilon} \mathcal{E}^{0}\left(\tau^{\prime}\right) d \tau^{\prime}\right\|_{\mathfrak{G} \rightarrow \mathfrak{G}}^{p^{\prime}} d y\right)^{1 / p^{\prime}} \\
& =\|\mathbf{F}\|_{\mathcal{H}_{p}} \varepsilon^{-3}\left(\int _ { 0 } ^ { \varepsilon ^ { 2 } } \left(\int_{0}^{y} \| T_{\varepsilon}^{*} \mathcal{E}^{0}\left(\varepsilon^{-2}\left(y-\tau^{\prime}\right)\right) b(\mathbf{D})^{*}\right.\right. \\
& \left.\left.\quad \times L_{Q}(\mathbf{D}) b(\mathbf{D}) \Pi \mathcal{E}^{0}\left(\varepsilon^{-2} \tau^{\prime}\right) T_{\varepsilon} \|_{\mathfrak{G} \rightarrow \mathfrak{G}} d \tau^{\prime}\right)^{p^{\prime}} d y\right)^{1 / p^{\prime}} \\
& \leq\|\mathbf{F}\|_{\mathcal{H}_{p}}\|f\|_{L_{\infty}}^{4}\left\|b(\mathbf{D})^{*} L_{Q}(\mathbf{D}) b(\mathbf{D}) \Pi\right\|_{\mathfrak{G} \rightarrow \mathfrak{G}} \varepsilon^{2 / p^{\prime}-1} .
\end{aligned}
$$

Estimate (5.11) and the relation

$$
b(\mathbf{D})^{*} L_{Q}(\mathbf{D}) b(\mathbf{D}) \Pi=\mathcal{U}^{-1}\left(\int_{\widetilde{\Omega}} \oplus \hat{N}_{Q}(\mathbf{k}) d \mathbf{k}\right) \mathcal{U}
$$

(see (5.9)) imply that

$$
\begin{aligned}
\left\|b(\mathbf{D})^{*} L_{Q}(\mathbf{D}) b(\mathbf{D}) \Pi\right\|_{\mathfrak{G} \rightarrow \mathfrak{G}} & =\sup _{\mathbf{k} \in \widetilde{\Omega}}\left\|\hat{N}_{Q}(\mathbf{k})\right\|_{L_{2}(\Omega) \rightarrow L_{2}(\Omega)} \\
& \leq r_{1}^{3} \sup _{|\boldsymbol{\theta}|=1}\left\|\hat{N}_{Q}(\boldsymbol{\theta})\right\|_{L_{2}(\Omega) \rightarrow L_{2}(\Omega)} \leq \mu_{2},
\end{aligned}
$$


Thus, the replacement of the upper limit of integration by $\tau$ in the second and third terms in (10.10) is possible if we add terms of order of $\varepsilon^{2 / p^{\prime}}$.

Now we consider the second term on the right-hand side of (10.9):

$$
\begin{aligned}
\int_{\tau-\varepsilon^{2}}^{\tau} & f^{\varepsilon} e^{-\mathcal{A}_{\varepsilon}(\tau-\widetilde{\tau})}\left(f^{\varepsilon}\right)^{*} \mathbf{F}(\cdot, \widetilde{\tau}) d \widetilde{\tau} \\
= & \int_{\tau-\varepsilon^{2}}^{\tau} \mathcal{E}^{0}(\tau-\widetilde{\tau}) \mathbf{F}(\cdot, \widetilde{\tau}) d \widetilde{\tau} \\
& +\int_{\tau-\varepsilon^{2}}^{\tau}\left(f^{\varepsilon} e^{-\mathcal{A}_{\varepsilon}(\tau-\widetilde{\tau})}\left(f^{\varepsilon}\right)^{*}-\mathcal{E}^{0}(\tau-\widetilde{\tau})\right) \mathbf{F}(\cdot, \tilde{\tau}) d \widetilde{\tau}
\end{aligned}
$$

To estimate the second term on the right in (10.18), we employ (8.1):

$$
\begin{gathered}
\left\|\int_{\tau-\varepsilon^{2}}^{\tau}\left(f^{\varepsilon} e^{-\mathcal{A}_{\varepsilon}(\tau-\widetilde{\tau})}\left(f^{\varepsilon}\right)^{*}-\mathcal{E}^{0}(\tau-\widetilde{\tau})\right) \mathbf{F}(\cdot, \tilde{\tau}) d \widetilde{\tau}\right\|_{\mathfrak{G}} \\
\quad \leq \mathfrak{C}\|\mathbf{F}\|_{\mathcal{H}_{p}}\left\|Q^{-1}\right\|_{L_{\infty}}\left(\int_{0}^{\varepsilon^{2}}\left(\frac{\varepsilon}{\sqrt{y+\varepsilon^{2}}}\right)^{p^{\prime}} d y\right)^{1 / p^{\prime}} \\
\quad=\mathfrak{C}\|\mathbf{F}\|_{\mathcal{H}_{p}}\left\|Q^{-1}\right\|_{L_{\infty}} \varepsilon^{2 / p^{\prime}}\left(\frac{2^{1-p^{\prime} / 2}-1}{1-p^{\prime} / 2}\right)^{1 / p^{\prime}}
\end{gathered}
$$

The case where $p=\infty$ is also included and corresponds to $p^{\prime}=1$.

We substitute formulas (10.7) and (10.9) in (10.5), and take into account formulas (10.10), (10.14), (10.16), (10.18), (10.19), and (10.6), as well as estimates (10.8), (10.12), (10.13), (10.15), and (10.17). Then, for $\tau>\varepsilon^{2}$ and $2<p \leq \infty$, we have

$$
\begin{aligned}
\mathbf{u}_{\varepsilon}=\mathbf{u}_{0} & +\varepsilon\left(\Lambda_{Q}^{\varepsilon} b(\mathbf{D}) \Pi_{\varepsilon} \mathbf{u}_{0}+\mathcal{E}^{0}(\tau)\left(\Lambda_{Q}^{\varepsilon} b(\mathbf{D}) \Pi_{\varepsilon}\right)^{*} \phi\right) \\
& -\varepsilon \int_{0}^{\tau} \mathcal{E}^{0}(\tau-\widetilde{\tau}) b(\mathbf{D})^{*} L_{Q}(\mathbf{D}) b(\mathbf{D}) \Pi_{\varepsilon} \mathcal{E}^{0}(\widetilde{\tau}) \phi d \widetilde{\tau} \\
& +\varepsilon \int_{0}^{\tau} \mathcal{E}^{0}(\tau-\widetilde{\tau})\left(\Lambda_{Q}^{\varepsilon} b(\mathbf{D}) \Pi_{\varepsilon}\right)^{*} \mathbf{F}(\cdot, \widetilde{\tau}) d \widetilde{\tau} \\
& -\varepsilon \int_{0}^{\tau} d \widetilde{\tau} \int_{0}^{\tau-\widetilde{\tau}} \mathcal{E}^{0}\left(\tau-\widetilde{\tau}-\tau^{\prime}\right) b(\mathbf{D})^{*} L_{Q}(\mathbf{D}) b(\mathbf{D}) \Pi_{\varepsilon} \mathcal{E}^{0}\left(\tau^{\prime}\right) \mathbf{F}(\cdot, \widetilde{\tau}) d \tau^{\prime}+\widetilde{\mathbf{u}}
\end{aligned}
$$

where

$$
\begin{aligned}
& \|\widetilde{\mathbf{u}}\|_{\mathfrak{G}} \leq \mathcal{C}_{1} \varepsilon^{2}\left(\tau+\varepsilon^{2}\right)^{-1}\|\phi\|_{\mathfrak{G}}+\mu(p, \varepsilon, \tau)\|\mathbf{F}\|_{\mathcal{H}_{p}} \varepsilon^{2 / p^{\prime}}, \quad \tau>\varepsilon^{2},
\end{aligned}
$$

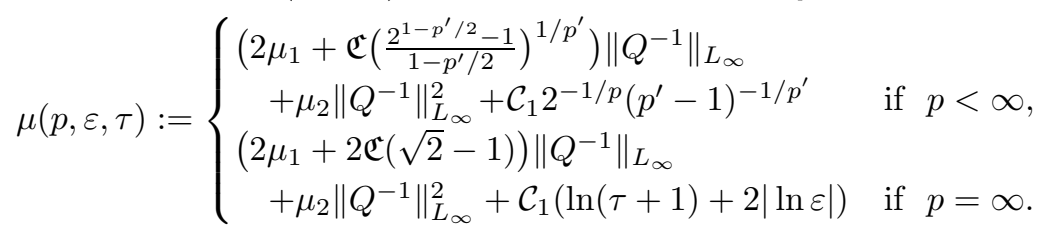

Here $p^{\prime}=p /(p-1)$, the constants $\mu_{1}$ and $\mu_{2}$ are defined in (10.15) and (10.17), $\mathcal{C}_{1}$ is defined in (7.26), and $\mathfrak{C}$ is the constant occurring in [15, (6.11)]. Note that $\mu(p, \varepsilon, \tau)$ in (10.22) depends on $\varepsilon$ and $\tau$ only if $p=\infty$. 
We show that the condition $\tau>\varepsilon^{2}$ mentioned above is not essential. Suppose $\tau \leq \varepsilon^{2}$. Then, by (10.5)-(10.7), we have

$$
\begin{aligned}
\mathbf{u}_{\varepsilon}= & \mathbf{u}_{0}+\varepsilon\left(\Lambda_{Q}^{\varepsilon} b(\mathbf{D}) \Pi_{\varepsilon} \mathcal{E}^{0}(\tau) \phi+\mathcal{E}^{0}(\tau)\left(\Lambda_{Q}^{\varepsilon} b(\mathbf{D}) \Pi_{\varepsilon}\right)^{*} \phi\right)+Y_{1}(\tau ; \varepsilon) \phi \\
& -\varepsilon \int_{0}^{\tau} \mathcal{E}^{0}(\tau-\widetilde{\tau}) b(\mathbf{D})^{*} L_{Q}(\mathbf{D}) b(\mathbf{D}) \Pi_{\varepsilon} \mathcal{E}^{0}(\widetilde{\tau}) \boldsymbol{\phi} d \widetilde{\tau} \\
& +\int_{0}^{\tau}\left(f^{\varepsilon} e^{-\mathcal{A}_{\varepsilon}(\tau-\widetilde{\tau})}\left(f^{\varepsilon}\right)^{*}-\mathcal{E}^{0}(\tau-\widetilde{\tau})\right) \mathbf{F}(\cdot, \widetilde{\tau}) d \widetilde{\tau}
\end{aligned}
$$

Comparing this with (10.20), we see that for $\tau \leq \varepsilon^{2}$ the solution $\widetilde{\mathbf{u}}$ can be represented in the form

$$
\begin{aligned}
\widetilde{\mathbf{u}}= & Y_{1}(\tau ; \varepsilon) \phi+\int_{0}^{\tau}\left(f^{\varepsilon} e^{-\mathcal{A}_{\varepsilon}(\tau-\widetilde{\tau})}\left(f^{\varepsilon}\right)^{*}-\mathcal{E}^{0}(\tau-\widetilde{\tau})\right) \mathbf{F}(\cdot, \widetilde{\tau}) d \widetilde{\tau} \\
& -\varepsilon \int_{0}^{\tau}\left(\mathcal{E}^{0}(\tau-\widetilde{\tau})\left(\Lambda_{Q}^{\varepsilon} b(\mathbf{D}) \Pi_{\varepsilon}\right)^{*}+\Lambda_{Q}^{\varepsilon} b(\mathbf{D}) \Pi_{\varepsilon} \mathcal{E}^{0}(\tau-\widetilde{\tau})\right) \mathbf{F}(\cdot, \widetilde{\tau}) d \widetilde{\tau} \\
& +\varepsilon \int_{0}^{\tau} d \widetilde{\tau} \int_{0}^{\tau-\widetilde{\tau}} \mathcal{E}^{0}\left(\tau-\widetilde{\tau}-\tau^{\prime}\right) b(\mathbf{D})^{*} L_{Q}(\mathbf{D}) b(\mathbf{D}) \Pi_{\varepsilon} \mathcal{E}^{0}\left(\tau^{\prime}\right) \mathbf{F}(\cdot, \widetilde{\tau}) d \tau^{\prime}
\end{aligned}
$$

Now we estimate $\|\widetilde{\mathbf{u}}\|_{\mathfrak{G}}$ in the case where $\tau \leq \varepsilon^{2}$. First, we use (10.8). Estimating the second term, we argue as in the proof of (10.19). Estimating the third term, we act by analogy with (10.14) and use (10.15). The last term can be treated by analogy with (10.16), taking (10.17) into account. Then

$$
\begin{aligned}
& \|\widetilde{\mathbf{u}}\|_{\mathfrak{G}} \leq\left\|Y_{1}(\tau ; \varepsilon) \phi\right\|_{\mathfrak{G}}+\|\mathbf{F}\|_{\mathcal{H}_{p}}\left(\int_{0}^{\varepsilon^{2}}\left\|f^{\varepsilon} e^{-\mathcal{A}_{\varepsilon}(\tau-\widetilde{\tau})}\left(f^{\varepsilon}\right)^{*}-\mathcal{E}^{0}(\tau-\widetilde{\tau})\right\|_{\mathfrak{G} \rightarrow \mathfrak{G}}^{p^{\prime}} d \widetilde{\tau}\right)^{1 / p^{\prime}} \\
& +\varepsilon\|\mathbf{F}\|_{\mathcal{H}_{p}}\left(\int_{0}^{\varepsilon^{2}}\left\|\mathcal{E}^{0}(\tau-\widetilde{\tau})\left(\Lambda_{Q}^{\varepsilon} b(\mathbf{D}) \Pi_{\varepsilon}\right)^{*}+\Lambda_{Q}^{\varepsilon} b(\mathbf{D}) \Pi_{\varepsilon} \mathcal{E}^{0}(\tau-\widetilde{\tau})\right\|_{\mathfrak{G} \rightarrow \mathfrak{G}}^{p^{\prime}} d \widetilde{\tau}\right)^{1 / p^{\prime}} \\
& +\varepsilon\|\mathbf{F}\|_{\mathcal{H}_{p}} \\
& \times\left(\int_{0}^{\varepsilon^{2}} d \widetilde{\tau}\left(\int_{0}^{\varepsilon^{2}-\widetilde{\tau}}\left\|\mathcal{E}^{0}\left(\tau-\widetilde{\tau}-\tau^{\prime}\right) b(\mathbf{D})^{*} L_{Q}(\mathbf{D}) b(\mathbf{D}) \Pi_{\varepsilon} \mathcal{E}^{0}\left(\tau^{\prime}\right)\right\|_{\mathfrak{G} \rightarrow \mathfrak{G}} d \tau^{\prime}\right)^{p^{\prime}}\right)^{1 / p^{\prime}} \\
& \leq \mathcal{C}_{1} \varepsilon^{2}\left(\tau+\varepsilon^{2}\right)^{-1}\|\phi\|_{\mathfrak{G}} \\
& +\|\mathbf{F}\|_{\mathcal{H}_{p}}\left\|Q^{-1}\right\|_{L_{\infty}} \varepsilon^{2 / p^{\prime}}\left(\mathfrak{C}\left(\frac{2^{1-p^{\prime} / 2}-1}{1-p^{\prime} / 2}\right)^{1 / p^{\prime}}+\mu_{2}\left\|Q^{-1}\right\|_{L_{\infty}}+2 \mu_{1}\right) \\
& \leq \mathcal{C}_{1} \varepsilon^{2}\left(\tau+\varepsilon^{2}\right)^{-1}\|\phi\|_{\mathfrak{G}}+\mu(p, \varepsilon, \tau)\|\mathbf{F}\|_{\mathcal{H}_{p}} \varepsilon^{2 / p^{\prime}}, \quad \tau \leq \varepsilon^{2},
\end{aligned}
$$

where $\mu(p, \varepsilon, \tau)$ is the quantity defined in (10.22). Thus, (10.20) turns out to be valid for any $\tau \in(0, T), 0<\varepsilon \leq 1,2<p \leq \infty$, with estimate (10.21).

As was noted above, taking account of the corrector in the first term on the right in (10.5) leads to refinement of approximation in comparison with (10.3) for any $1<p \leq \infty$. In the second term on the right in (10.5), taking account of the corrector leads to such refinement only in the case where $2<p \leq \infty$. Finally, we arrive at the following conclusion.

Theorem 10.1. Suppose $0<\varepsilon \leq 1, T>0, \tau \in(0, T), \mathbf{u}_{\varepsilon}$ is the solution of problem (10.1), and $\mathbf{u}_{0}$ is the solution of problem (10.2). Also, suppose that $\phi \in L_{2}\left(\mathbb{R}^{d} ; \mathbb{C}^{n}\right)$ and 
$\mathbf{F} \in L_{p}\left((0, T) ; L_{2}\left(\mathbb{R}^{d} ; \mathbb{C}^{n}\right)\right)$. Then for $2<p \leq \infty$, we have

$$
\begin{aligned}
\mathbf{u}_{\varepsilon}= & \mathbf{u}_{0}+\varepsilon\left(\Lambda_{Q}^{\varepsilon} b(\mathbf{D}) \Pi_{\varepsilon} \mathbf{u}_{0}+\mathcal{E}^{0}(\tau)\left(\Lambda_{Q}^{\varepsilon} b(\mathbf{D}) \Pi_{\varepsilon}\right)^{*} \boldsymbol{\phi}\right) \\
& -\varepsilon \int_{0}^{\tau} \mathcal{E}^{0}(\tau-\widetilde{\tau}) b(\mathbf{D})^{*} L_{Q}(\mathbf{D}) b(\mathbf{D}) \Pi_{\varepsilon} \mathcal{E}^{0}(\widetilde{\tau}) \phi d \widetilde{\tau} \\
& +\varepsilon \int_{0}^{\tau} \mathcal{E}^{0}(\tau-\widetilde{\tau})\left(\Lambda_{Q}^{\varepsilon} b(\mathbf{D}) \Pi_{\varepsilon}\right)^{*} \mathbf{F}(\cdot, \widetilde{\tau}) d \widetilde{\tau} \\
& -\varepsilon \int_{0}^{\tau} d \widetilde{\tau} \int_{0}^{\tau-\widetilde{\tau}} \mathcal{E}^{0}\left(\tau-\widetilde{\tau}-\tau^{\prime}\right) b(\mathbf{D})^{*} L_{Q}(\mathbf{D}) b(\mathbf{D}) \Pi_{\varepsilon} \mathcal{E}^{0}\left(\tau^{\prime}\right) \mathbf{F}(\cdot, \widetilde{\tau}) d \tau^{\prime}+\widetilde{\mathbf{u}},
\end{aligned}
$$

where

$$
\|\widetilde{\mathbf{u}}\|_{\mathfrak{G}} \leq \mathcal{C}_{1} \varepsilon^{2}\left(\tau+\varepsilon^{2}\right)^{-1}\|\boldsymbol{\phi}\|_{\mathfrak{G}}+\mu(p, \varepsilon, \tau)\|\mathbf{F}\|_{\mathcal{H}_{p}} \varepsilon^{2 / p^{\prime}}
$$

$\mathcal{C}_{1}$ is the constant defined in (7.26), and $\mu(p, \varepsilon, \tau)$ is defined in (10.22). For $1<p \leq 2$ we have

$$
\begin{aligned}
\mathbf{u}_{\varepsilon}= & \mathbf{u}_{0}+\varepsilon\left(\Lambda_{Q}^{\varepsilon} b(\mathbf{D}) \Pi_{\varepsilon} \mathcal{E}^{0}(\tau) \boldsymbol{\phi}+\mathcal{E}^{0}(\tau)\left(\Lambda_{Q}^{\varepsilon} b(\mathbf{D}) \Pi_{\varepsilon}\right)^{*} \boldsymbol{\phi}\right) \\
& -\varepsilon \int_{0}^{\tau} \mathcal{E}^{0}(\tau-\widetilde{\tau}) b(\mathbf{D})^{*} L_{Q}(\mathbf{D}) b(\mathbf{D}) \Pi_{\varepsilon} \mathcal{E}^{0}(\widetilde{\tau}) \boldsymbol{\phi} d \widetilde{\tau}+\widetilde{\mathbf{u}}
\end{aligned}
$$

where

$$
\|\widetilde{\mathbf{u}}\|_{\mathfrak{G}} \leq \mathcal{C}_{1} \varepsilon^{2}\left(\tau+\varepsilon^{2}\right)^{-1}\|\phi\|_{\mathfrak{G}}+\mathfrak{C}\left\|Q^{-1}\right\|_{L_{\infty}}\|\mathbf{F}\|_{\mathcal{H}_{p}} \theta(p, \varepsilon, \tau) \varepsilon
$$

with $\theta(p, \varepsilon, \tau)$ as defined in (10.4).

Note that in estimate (10.23), the best order is achieved if $p=\infty$. The coefficient of $\|F\|_{\mathcal{H}_{\infty}}$ is then $O\left(\varepsilon^{2}|\ln \varepsilon|\right)$.

10.2. The case where $f=\mathbf{1}_{n}$. Consider the following nonhomogeneous Cauchy problem:

$$
\frac{\partial \widehat{\mathbf{u}}_{\varepsilon}(\mathbf{x}, \tau)}{\partial \tau}=-\widehat{\mathcal{A}}_{\varepsilon} \widehat{\mathbf{u}}_{\varepsilon}(\mathbf{x}, \tau)+\mathbf{F}(\mathbf{x}, \tau), \quad \widehat{\mathbf{u}}_{\varepsilon}(\mathbf{x}, 0)=\phi(\mathbf{x}) .
$$

Let $\widehat{\mathbf{u}}_{0}$ be the solution of the "homogenized" problem

$$
\frac{\partial \widehat{\mathbf{u}}_{0}(\mathbf{x}, \tau)}{\partial \tau}=-\widehat{\mathcal{A}}^{0} \widehat{\mathbf{u}}_{0}(\mathbf{x}, \tau)+\mathbf{F}(\mathbf{x}, \tau), \quad \widehat{\mathbf{u}}_{0}(\mathbf{x}, 0)=\phi(\mathbf{x}) .
$$

Here the operators $\widehat{\mathcal{A}}_{\varepsilon}, \widehat{\mathcal{A}}^{0}$ are the same as in Subsection 9.2. Suppose that the functions $\phi(\mathbf{x}), \mathbf{F}(\mathbf{x}, \tau)$ satisfy the conditions of Subsection 10.1. We formulate Theorem 10.1 for this case.

Theorem 10.2. Suppose $0<\varepsilon \leq 1, T>0, \tau \in(0, T)$, $\widehat{\mathbf{u}}_{\varepsilon}$ is the solution of problem (10.24), and $\widehat{\mathbf{u}}_{0}$ is the solution of problem $(10.25)$. Also, suppose that $\phi \in L_{2}\left(\mathbb{R}^{d} ; \mathbb{C}^{n}\right)$ and $\mathbf{F} \in L_{p}\left((0, T) ; L_{2}\left(\mathbb{R}^{d} ; \mathbb{C}^{n}\right)\right)$. Then for $2<p \leq \infty$ we have

$$
\begin{aligned}
\widehat{\mathbf{u}}_{\varepsilon}= & \widehat{\mathbf{u}}_{0}+\varepsilon\left(\Lambda^{\varepsilon} b(\mathbf{D}) \Pi_{\varepsilon} \widehat{\mathbf{u}}_{0}+e^{-\hat{\mathcal{A}}^{0} \tau}\left(\Lambda^{\varepsilon} b(\mathbf{D}) \Pi_{\varepsilon}\right)^{*} \phi\right) \\
& -\varepsilon \int_{0}^{\tau} e^{-\hat{\mathcal{A}}^{0}(\tau-\widetilde{\tau})} b(\mathbf{D})^{*} L(\mathbf{D}) b(\mathbf{D}) \Pi_{\varepsilon} e^{-\hat{\mathcal{A}}^{0} \widetilde{\tau}} \phi d \widetilde{\tau} \\
& +\varepsilon \int_{0}^{\tau} e^{-\hat{\mathcal{A}}^{0}(\tau-\widetilde{\tau})}\left(\Lambda^{\varepsilon} b(\mathbf{D}) \Pi_{\varepsilon}\right)^{*} \mathbf{F}(\cdot, \widetilde{\tau}) d \widetilde{\tau} \\
& -\varepsilon \int_{0}^{\tau} d \widetilde{\tau} \int_{0}^{\tau-\widetilde{\tau}} e^{-\hat{\mathcal{A}}^{0}\left(\tau-\widetilde{\tau}-\tau^{\prime}\right)} b(\mathbf{D})^{*} L(\mathbf{D}) b(\mathbf{D}) \Pi_{\varepsilon} e^{-\hat{\mathcal{A}}^{0} \tau^{\prime}} \mathbf{F}(\cdot, \widetilde{\tau}) d \tau^{\prime}+\widetilde{\mathbf{u}},
\end{aligned}
$$


where

$$
\|\widetilde{\mathbf{u}}\|_{\mathfrak{G}} \leq \widehat{\mathcal{C}}_{1} \varepsilon^{2}\left(\tau+\varepsilon^{2}\right)^{-1}\|\phi\|_{\mathfrak{G}}+\widehat{\mu}(p, \varepsilon, \tau)\|\mathbf{F}\|_{\mathcal{H}_{p}} \varepsilon^{2 / p^{\prime}} .
$$

For $1<p \leq 2$ we have

$$
\begin{aligned}
\widehat{\mathbf{u}}_{\varepsilon}= & \widehat{\mathbf{u}}_{0}+\varepsilon\left(\Lambda^{\varepsilon} b(\mathbf{D}) \Pi_{\varepsilon} e^{-\hat{\mathcal{A}}^{0} \tau} \boldsymbol{\phi}+e^{-\hat{\mathcal{A}}^{0} \tau}\left(\Lambda^{\varepsilon} b(\mathbf{D}) \Pi_{\varepsilon}\right)^{*} \boldsymbol{\phi}\right) \\
& -\varepsilon \int_{0}^{\tau} e^{-\hat{\mathcal{A}}^{0}(\tau-\widetilde{\tau})} b(\mathbf{D})^{*} L(\mathbf{D}) b(\mathbf{D}) \Pi_{\varepsilon} e^{-\hat{\mathcal{A}}^{0} \widetilde{\tau}} \boldsymbol{\phi} d \widetilde{\tau}+\widetilde{\mathbf{u}},
\end{aligned}
$$

where

$$
\|\widetilde{\mathbf{u}}\|_{\mathfrak{G}} \leq \widehat{\mathcal{C}}_{1} \varepsilon^{2}\left(\tau+\varepsilon^{2}\right)^{-1}\|\phi\|_{\mathfrak{G}}+\widehat{\mathfrak{C}}\|\mathbf{F}\|_{\mathcal{H}_{p}} \theta(p, \varepsilon, \tau) \varepsilon .
$$

The constant $\widehat{\mathfrak{C}}$ is defined in [15, (6.7)], $\theta(p, \varepsilon, \tau)$ is defined in (10.4), and the constants $\hat{\mathcal{C}}_{1}$ and $\widehat{\mu}(p, \varepsilon, \tau)$ can be obtained from (7.26) and (10.22) (respectively) by putting $\|f\|_{L_{\infty}}=$ $\left\|f^{-1}\right\|_{L_{\infty}}=\|Q\|_{L_{\infty}}=\left\|Q^{-1}\right\|_{L_{\infty}}=1$.

\section{REFERENCES}

[1] N. S. Bakhavalov and G. P. Panasenko, Homogenization: Averaging processes in periodic media, Mathematical Problems in the Mechanics of Composite Materials, Nauka, Moscow, 1984; English transl., Math. Appl. (Soviet Ser.), vol. 36, Kluwer Acad Publ. Group, Dordrecht, 1989. MR0797571 (86m:73049), MR1112788(92d:73002)

[2] A. Bensoussan, J.-L. Lions, and G. Papanicolaou, Asymptotic analysis for periodic structures, Stud. Math. Appl., vol. 5, North-Holland Publ. Co., Amsterdam-New York, 1978, 700 pp. MR0503330 (82h:35001)

[3] M. Sh. Birman and T. A. Suslina, Threshold effects near the lower edge of the spectrum for periodic differential operators of mathematical physics, Systems, Approximation, Singular Integral Operators, and Related Topics (Bordeaux, 2000), Oper. Theory Adv. Appl., vol. 129, Birkhäuser, Basel, 2001, pp. 71-107. MR1882692 (2003f:35220)

[4] - Second order periodic differential operators. Threshold properties and homogenization, Algebra i Analiz 15 (2003), no. 5, 1-108; English transl., St. Petersburg Math. J. 15 (2004), no. 5, 639-714. MR 2068790 (2005k:47097)

[5] - Threshold approximations with corrector for the resolvent of a factorized selfadjoint operator family, Algebra i Analiz 17 (2005), no. 5, 69-90; English transl., St. Petersburg Math. J. 17 (2006), no. 5, 745-762. MR2241423(2008d:47047)

[6] - Homogenization with corrector term for periodic elliptic differential operators, Algebra i Analiz 17 (2005), no. 6, 1-104; English transl., St. Petersburg Math. J. 17 (2006), no. 6, 897-973. MR2202045 (2006k:35011)

[7] Homogenization with corrector term for periodic differential operators. Approximation of solutions in the Sobolev class $H^{1}\left(\mathbb{R}^{d}\right)$, Algebra i Analiz 18 (2006), no. 6, 1-130; English transl., St. Petersburg Math. J. 18 (2007), no. 6, 857-955. MR2307356 (2008d:35008)

[8] V. V. Zhikov, Spectral approach to asymptotic diffusion problems, Differentsial'nye Uravneniya 25 (1989), no 1, 44-50; English transl., Differential Equations 25 (1989), no. 1, 33-39. MR0986395 (90a:35107)

[9] , On some estimates from homogenization theory, Dokl. Ros. Akad. Nauk 406 (2006), no. 5, 597-601; English transl. in Dokl. Math. MR2347318 (2008d:35018)

[10] V. V. Zhikov, S. M. Kozlov, and O. A. Oleĭnik, Homogenization of differential operators, Nauka, Moscow, 1993; English transl., Springer-Verlag, Berlin, 1994. MR1318242 (96h:35003a) MR $1329546(96 \mathrm{~h}: 35003 \mathrm{~b})$

[11] V. V. Zhikov and S. E. Pastukhova, On operator estimates for some problems in homogenization theory, Russ. J. Math. Phys. 12 (2005), no. 4, 515-524. (English) MR2201316 (2007c:35014)

[12] _ Estimates of homogenization for a parabolic equation with periodic coefficients, Russ. J. Math. Phys. 13 (2006), no. 2, 224-237. (English) MR 2262826 (2007k:35025)

[13] O. A. Ladyzhenskaya and N. N. Ural'tseva, Linear and quasilinear equations of elliptic type, Nauka, Moscow, 1964; English transl., Acad. Press, New York-London, 1968. MR0211073 (35:1955) MR0244627 (39:5941) 
[14] T. A. Suslina, On the homogenization of periodic parabolic systems, Funktsional. Anal. i Prilozhen. 38 (2004), no. 4, 86-90; English transl., Funct. Anal. Appl. 38 (2004), no. 4, 309-312. MR2117512 (2005j:35008)

[15] - Homogenization of a periodic parabolic Cauchy problem, Nonlinear Equations and Spectral Theory, Amer. Math. Soc. Transl. (2), vol. 220, Amer. Math. Soc., Providence, RI, 2007, pp. 201233. MR2343612 (2008k:35030)

Department of Mathematics and Mechanics, St. Petersburg State University, UniverSitetskil Prospekt 28, Petrodvorets, 198504 St. Petersburg, Russia

E-mail address: vasilevskaya-e@yandex.ru

Received 1/SEP/2009

Translated by THE AUTHOR 